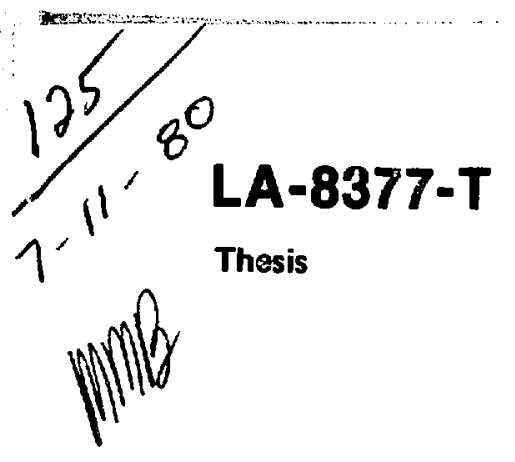

\title{
if. 1480
}

Measurement of the Doubly Differential

Cross Section for $\pi^{-} p \rightarrow \pi^{+} \pi^{-} n$

Near Threshold

竞 


\title{
Measurement of the Doubly Differential Cross Section for $\pi^{-} p \rightarrow \pi^{+} \pi^{-} n$ Near Threshold
}

\author{
John B. Walter*
}

"Present address: EG\&G Idaho, Inc., Idaho Falls, 1083415.

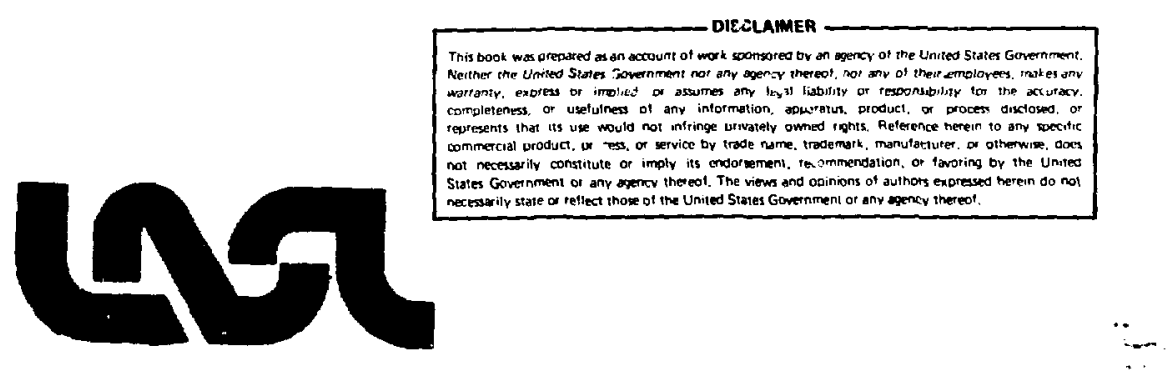




\section{TABLE OF CONTENTS}

ABSTRACT

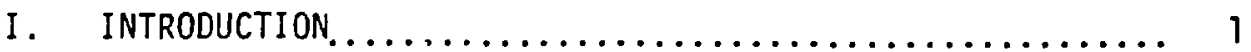

II. EXPERIMENTAL DESIGN AND EQUIPMENT............... 6

Introduction. ......................... 6

The Pion Beam.......................... 6

Liquid Hydrogen Target.......................

Spectrometer............................ 12

Detectors and Detection System................ 1 s

Beam Flux Monitors........................ 26

Data Acquisition.......................... 27

III. AUXILIARY EXPERIMENTS AND ANALYSES .............. 31

Calibration of Spectrometer Momentum Curve.......... 31

Detection Channel Efficiencies................ 36

Angular Survey of $\pi^{+} \mathrm{p}$ Elastic Scattering............. 42

$\pi^{-} \mathbf{p}$ Elastic Scattering...................... 51

IV. INTERPOLATION OF THE $\pi \pm p$ ELASTIC DIFFERENTIAL CROSS

SECTIONS ........................... 67

Interpolation Below $300 \mathrm{MeV} . \ldots \ldots \ldots \ldots \ldots \ldots \ldots \ldots \ldots \ldots$

Interpolation at $330 \mathrm{MeV}$ and $356 \mathrm{MeV} \ldots \ldots \ldots \ldots \ldots 77$

v. ANALYSIS OF $\pi^{+}$PRODUCTION................... 84

Doubly Differential Cross Sections................ 84

Event rate.......................... 84

Matrix element....................... 86

Doubly differential cross section............. 88 
Integrated Cross Sections...................... 89

Comparison To Soft Pion Theory.................. 104

VI. SUMMARY AND CONCLUSIONS................... 109

ACKNOWLEDGEMENTS .......................... 110

APPENDIX A. NONLINEAR LEAST SQUARES FITTING ALGORITHM...... 117

APPENC IX B. MOMENTUM CORRECTIONS................ 113

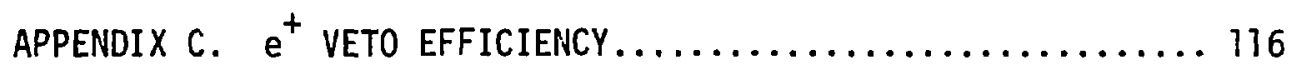

REFERENCES ............................. 119 


\section{LIST OF TABLES}

I. Ihreshold matri elements ........... 2

II. Spectrometer properties . . . . . . . . . . 15

III. Parameter values for spectrometer excitation function . . 17

IV. Drtection channel efficiencies .......... 19

v. juandities scaled during a run ............29

VI. Results of ${ }^{241} \mathrm{Am}$ data............... 35

VII. Anqular survey of $\pi^{+} p \rightarrow \pi^{+} p$ at $140 \mathrm{MeV} \ldots . . . . . .48$

VIII. Repeat angular survey of $\pi^{+} p \rightarrow \pi^{+} p$ at $140 \mathrm{MeV}$. . . . . 50

IX. Results from $\pi^{-} p \rightarrow \pi^{-} p$ at $229 \mathrm{MeV}$. . . . . . . . 59

X. Results from $\pi^{-} p \rightarrow \pi^{-} p$ at $254 \mathrm{MeV}$.......... 60

XI. Results from $\pi^{-} p \rightarrow \pi^{-} p$ at $279 \mathrm{MeV}$.......... 61

XII. Results from $\pi^{-} p \rightarrow \pi^{-} p$ at $292 \mathrm{MeV}$. . . . . . . . 62

XIII. Results from $\pi^{-} p \rightarrow \pi^{-} p$ at $330 \mathrm{MeV}$. . . . . . . . 63

XIV. Differential cross sections for $\pi^{-} p \rightarrow \pi^{-} p$ at $330 \mathrm{MeV} . .63$

XV. Results from $\pi^{-} p \rightarrow \pi^{-} p$ at $356 \mathrm{MeV}$.......... 64

XVI. Differential cross sections for $\pi^{-} p \rightarrow \pi^{-} p$ at $356 \mathrm{MeV}$. . 64

XVII. Results from the study of $\pi^{-} p \rightarrow \pi^{-} p \ldots . . . . . .65$

XVIII. Incident momentum distributions corrected for loss of energy in the target ............ 66

XIX. Real part of polynomial parameters for phase shifts . . . 75 
$X X$. Imaginary part of polynomial parameters for phase shifts . 76

$X X I$. Resonance parameters for $P_{11}$ and $P_{33}$ phase shifts. . . 76 XXII. Parameters for C-quantities ........... 78

XXIII. Doubly differential cross sections at $254 \mathrm{MeV}$. . . . . 90 XXIV. Doubly differential cross sections at $279 \mathrm{MeV}$. . . . . 91 XXV. Doubly differential cross sections at $292 \mathrm{MeV}$. . . . . 92 XXVI. Doubly differential cross sections at $330 \mathrm{MeV}$. . . . 93 XXVII. Doubly differential cross sections at $356 \mathrm{MeV}$...... . 94 XXVIII. Parameter values for $\left\langle|M|^{2}\right\rangle$ at each energy . . . . . . 101 XXIX. Integrated cross sections for $\pi^{-} p \rightarrow \pi^{+} \pi^{-} n \ldots 102$ $X X X$. Comparison to soft pion theory........... . 107 


\section{LIST OF FIGURES}

1 Plan view of experiment .......... . 7

2 Liquid hydrogen target flask . . . . . . 10

3 Target flask with reservoir and refrigerator. . 11

4 Profile of spectrometer and detector system . . 13

5 Profile of spectrometer .......... 14

6 Detection channel efficiencies . . . . . . 20

7 Ladder of surface barrier detectors . . . . . 22

8 Trigger and deadtime logic . . . . . . . 25

9 Sample ${ }^{241}$ Am spectrum for source extended horizontally............. . . 34

10 Sample spectra for channel efficiencies . . . 38

i1 Data for spectrometer dispersion . . . . . 41

12 Sample spectra for $\pi^{+} p \rightarrow \pi^{+} p$. . . . . . 46

13 Angular distribution of $\pi^{+} \mathrm{p} \rightarrow \pi^{+} \mathrm{p}$ at $140 \mathrm{MeV} . \quad 49$

14 Repeat of angular distribution of $\pi^{+} p \rightarrow \pi^{+} p$

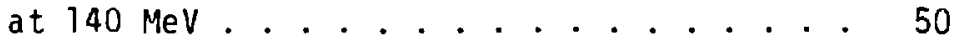

15 Sample spectra for $\pi^{-} p \rightarrow \pi^{-} p$ at $229 \mathrm{Mel}$. . . 53

16 Sample spectra for $\pi^{-} p \rightarrow \pi^{-} p$ at $254 \mathrm{MeV} . . .54$

17 Sample spectra for $\pi^{-} p \rightarrow \pi^{-} p$ at $279 \mathrm{MeV}$. . . 55

18 Sample spectra for $\pi^{-} p \rightarrow \pi^{-} p$ at $292 \mathrm{MeV}$. . . 56

19 Sample spectra for $\pi^{-} p \rightarrow \pi^{-} p$ at $330 \mathrm{MeV}$. . . 57

20 Sample spectra for $\pi^{-} p \rightarrow \pi^{-} p$ at $356 \mathrm{MeV}$. . . 58 
$21 \quad$ Angular distribution of $\pi^{-} p \rightarrow \pi^{-} p$ at $229 \mathrm{MeV}$. 59

22 Angular distribution of $\pi^{-} p \rightarrow \pi^{-} p$ at $254 \mathrm{MeV}$. 60

23 Angular distribution of $\pi^{-} p \rightarrow \pi^{-} p$ at $279 \mathrm{MeV}$. 61

24 Angular distribution of $\pi^{-} p \rightarrow \pi^{-} p$ at $292 \mathrm{MeV} .62$

25 Angular distributions of Bussey et al . . . 79

26 Angular distributions of Bussey et al . . . 79

27 Angular distributions of Bussey et al . . . 80

28 Angular distributions of Bussey et al . . . 80

29 Angular distribution of 0 gden et al at $370 \mathrm{MeV} 82$

30 Variation of the differential cross section

with incident energy . . . . . . 83

31 Values of $\left\langle|M|^{2}\right\rangle$ at $254 \mathrm{MeV}$....... . 95

32 Values of $\left\langle|M|^{2}\right\rangle$ at $279 \mathrm{MeV}$....... 96

33 Values of $\left\langle|M|^{2}\right\rangle$ at $292 \mathrm{MeV}$....... 97

34 Values of $\left\langle|M|^{2}\right\rangle$ at $330 \mathrm{MeV}$....... 98

35 Values of $\left\langle|\mathrm{M}|^{2}\right\rangle$ at $356 \mathrm{MeV}$....... 99

36 Integrated cross sections for $\pi^{-} p \rightarrow \pi^{+} \pi^{-} n$. 103

37 Extrapolation of $\overline{|M|^{2}}$ to threshold..... 105

38 Effect of the uncertainty in $\varepsilon \ldots 106$ 


\section{MEASUREMENT OF THE DOUBLY DIFFERENTIAL \\ CROSS SECTION FOR $\pi^{-} p \rightarrow \pi^{+} \pi^{-} n$ \\ NEAR THRESHOLD}

by

John B. Hàlter

\section{ABSTRACT}

The doubly differential cross sections for the $\pi^{+}$from the reaction $\pi^{-} p \rightarrow \pi^{+} \pi^{-} n$ were measured at about twenty points for each of five energies between $245 \mathrm{MeV}$ and $356 \mathrm{MeV}$. The experiment was carried out at the Clinton P. Anderson Meson Physics Facility, where a double focussing magnetic spectrometer detected the $\pi^{+}$mesons produced in a liquid hydrogen target. The measurements were normalized by comparison with $\pi^{-p} p$ elastic scattering measured with the same apparatus. These are the first such measurements in this energy range, and have an accuracy between $4.7 \%$ and $39 \%$. The integrated reaction cross section was determined at each energy with an accuracy of about $5 \%$. These agree with but are an improvement over previous measurements in this energy range. Compaison of the extrapolated threshold value of the mean square modulus of the matrix element with the soft pion caiculations favors the symmetry breaking mechanism of isinberg $(\xi=0)$. It also demonstrates the futility of attempting to determine the symmetry breaking parameter $\xi$ from a single measurement of the integrated reaction cross section. 


\section{CHAPTER I}

INTRODUCTION

The $\pi N$ and $\pi \pi$ interactions are of interest to the understanding of the strong interaction. Soft pion theory addresses itself to these interactions. A number of processes are determined with but a single parameter, the pion decay constant $f_{\pi}$, fermitting several evaluations of $f_{\pi}$. The lifetime of the charged pion, the Goldberger-Treiman relation, the $\pi \mathrm{N} s$-wave scattering lengths, the $\pi \pi \mathrm{p}$-wave isovector scattering length all yield values for $f_{\pi}$ consistent to within $8 \%$. This measure of success is striking in that the hypotheses involved are strictly valid only for soft pions; i.e., pions having vanishing four momenta. With this encouragement we examine soft pion theory further.

Sins? pion production in the reactions $\pi N \rightarrow \pi \pi N$ involves both the $\pi \mathrm{N}$ and $\pi \pi$ interactions. Considerations of parity and the Bose statistics of the pions require the threshold matrix elements for all the possible charge states to be calculable from two matrix elements. Assuming the current algebra hypothesis and the partial conservation of the axial vector current (PCAC), soft pion calculations determine both matrix elements with a single additional parameter $\xi$. This parameter distinguishes amongst the symmetry breaking mechanisms proposed to provide nonconservation of the axial vector current. Table 1 presents the threshold matrix elements predicted for the five charge states tractable to experiment, and the predicted threshold behavior of the cross section. Knowledge of the threshold matrix element for the first two and 
Table I. Threshold matrix elements in soft pion theory.

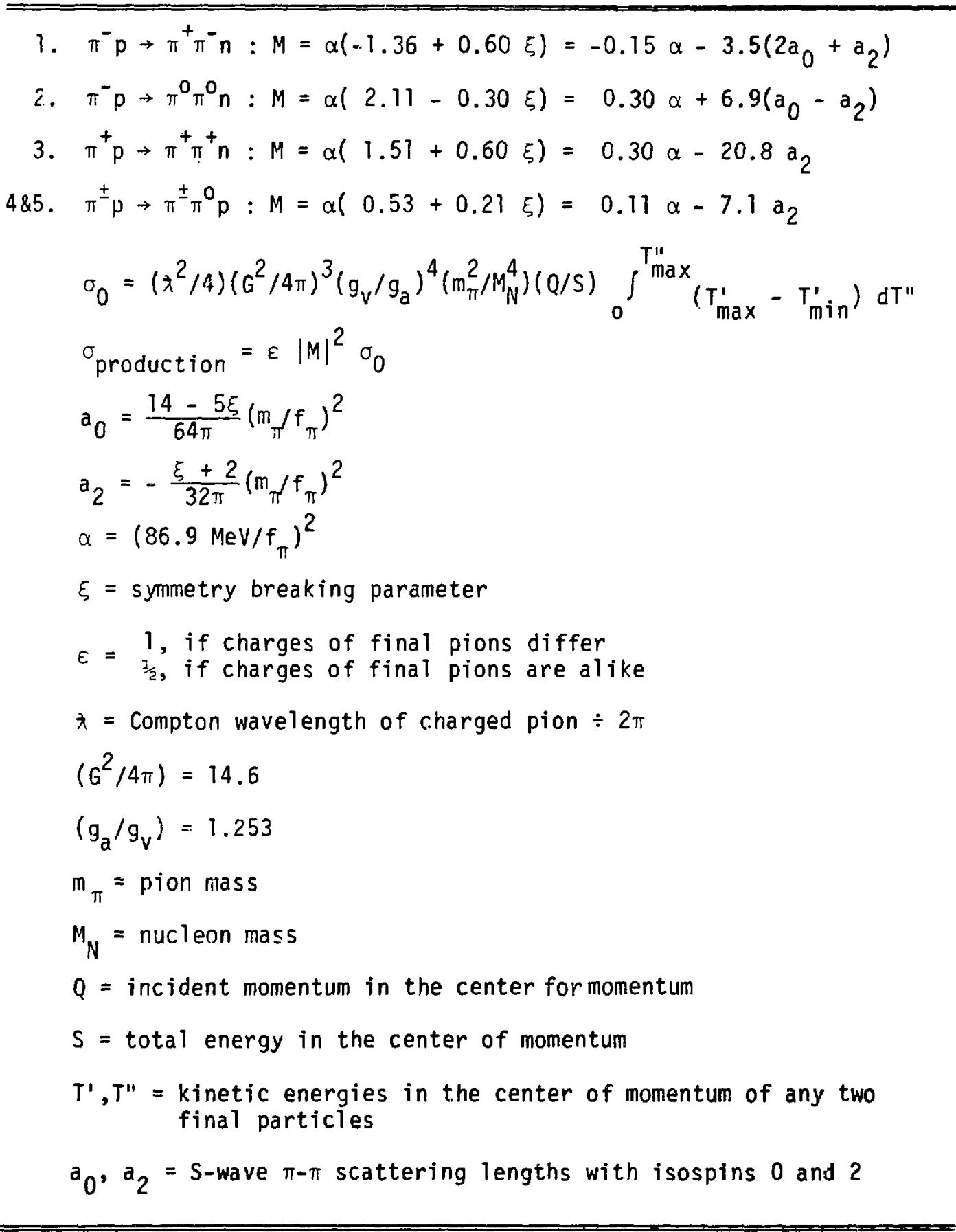


one of the last three would critically test this application of the theory. However, measurements of the small cross sections near threshold have been of limited accuracy for the first reactior, and totally lacking for the last four. Even for the first reaction, the required extrapolation of the matrix element to threshold has been less than convincing. None the less, experiment and calculation have agreed roughiy for $\xi$ between \pm 1 .

This work was planned as a study of the first reaction, $\pi-p \rightarrow \pi^{+} \pi-n$, with the improved precision possible with the intense pion beams available at the ilinton P. Anderson Meson Physics Facility (LAMPF). A double focusing, $180^{\circ}$ vertical bend, magnetic spectrometer measured the doubly differential cross sections for $\pi^{+}$mesons produced in a liquid hydrogen target. A ladder of nineteen surface barrier detectors spanning the focal plane covered a $\Delta p / p$ of $9.5 \%$, providing momentum resolution by virtue of spatial discrimination. The target flask, particle trajectory in the spectrometer, and the array of detectors were in a common vacuum to minimize loss of energy and multiple scatiering of lciw energy particles. Three plastic scintillation detectors, providing a trigger, and a threshold Cerenkov detector, providing electron discrimination, completed the detection system. The entire assembly, including the target and cryostat, could be rotated as a unit about the vertical axis of the cylindrical target to angles between $32^{\circ}$ and $130^{\circ}$. The spectrometer couid select charged particles with momenta up to $325 \mathrm{MeV} / \mathrm{C}$, and detect $\pi$ mesons down to $70 \mathrm{MeV} / \mathrm{c}$. The flux of incident $\pi$ mesons was measured by both an ion chamber and a scattering monitor. The complete apparatus was calibrated as a whole by detecting the $\pi$ meson elastically scattered 
from the hydrogen, and adjusting the overall normalization of the measurement of the $\pi p$ elastic cross sections.

The study included five incident energies, $254 \mathrm{MeV}, 279 \mathrm{MeV}, 292$ $\mathrm{MeV}, 330 \mathrm{MeV}$ and $356 \mathrm{MeV}$, with the express purpose of extrapolation to the $172 \mathrm{MeV}$ threshold and comparison to soft pion calculations. The doubly differential cross section, $\left(\frac{d^{2} \sigma}{\partial \Omega \delta p}\right)_{L A B}$, of the produced $\pi^{+}$meson was measured at about twenty points for each energy. In each case these measurements were distributed more or less uniformly in the center of momentum frame over the accessible portion of $(T, \cos \theta)$ space. At each point the square modulus of the matrix element, averaged over the unobserved variables, was determined from the measurement. For each energy a plausibie function of $T$ and $\cos \theta$ was fitted to the collection of square moduli corrected for coulomb attraction in the final state. This function, weighted by the density of phase space, was averaged over $T$ and $\cos \theta$ to provide the corrected average square moduius of the matrix element. The function was aiso multiplied by the Coulomb factor and the density of phase space and integrated over $T$ and $\cos \theta$ to determine the integrated reaction cross section. The threshold value of the mean corrected square modulus was extrapolated from these five values and a more recent value 3 at $230 \mathrm{MeV}$ and compared to the soft pion calculation to determine the symmetry breaking parameter $\xi$. The large variation with incident energy apparent in the mean corrected square modulus even at $230 \mathrm{MeV}$ demonstrated the necessity of extrapolating to threshold from a series of measurements. No single measurement which validly represented the soft pion limit would be practical.

The equipment and experimental procedure are detailed in Chapter II, 
while Chapter III describes the accompanying calibrations. Chapter IV delineates the interpolation of the differential cross section for $\pi p$ elastic scattering from the best measurements available. Chapter $v$ presents the analysis of the measurements for $\pi^{-} p \rightarrow \pi^{+} \pi^{-} n$. 


\section{CHAPTER II}

\section{EXPERIMENTAL DESIGN AND EQUIPMENT}

\section{Introduction}

This experiment measured the doubly differential cross section for the reaction $\pi^{-} p \rightarrow \pi^{+} \pi^{-n}$ by detecting only the outgo ..., $\pi^{+}$meson. The plan view of the experiment is shown in Fig. 1. A beam of $\pi^{-}$mesons was incident upon a liquid hydrogen target. A magnetic spectrometer passed $\pi^{+}$mesons of the selected angle and momentum, focusing them onto a ladder of surface barrier detectors. The $\pi^{+}$mesons continued through a trigger telescope of three plastic scintillation detectors, and a threshold Cerenkov detector. A scattering monitor downstream and an ion chamber upstream of the liquid hydrogen target measured the flux of $\pi^{-}$ mesons in the beam. The horizontal and vertical positions of the $\pi^{-}$ beam were monitored by a pair of multiwire proportional chambers.

\section{The Pion Beàn}

The experiment was carried out at the Clintor. $\mathrm{P}$. Anderson Meson Physics Facility (LAMPF) on the west branch of the secondary beam channel "Pion and Particle Physics" ( $\mathrm{P}^{3}$ - west). This channel could provide positive or negative $\pi$ miesons over a broad range of momenta, $190 \mathrm{MeV} / \mathrm{c}<$ $\mathrm{p}<730 \mathrm{MeV} / \mathrm{c}$, and a reasonable range of momentum bite, $0.0025<\Delta \mathrm{p} / \mathrm{p}<$ $0.10{ }^{4}$ It had been designed to be both isochronous and achromatic, which means that the channel preserves the time structure of the beam and that the position and angle of a $\pi$ meson arriving at the target are independent of the particle momentum. The channel was tuned to deliver 


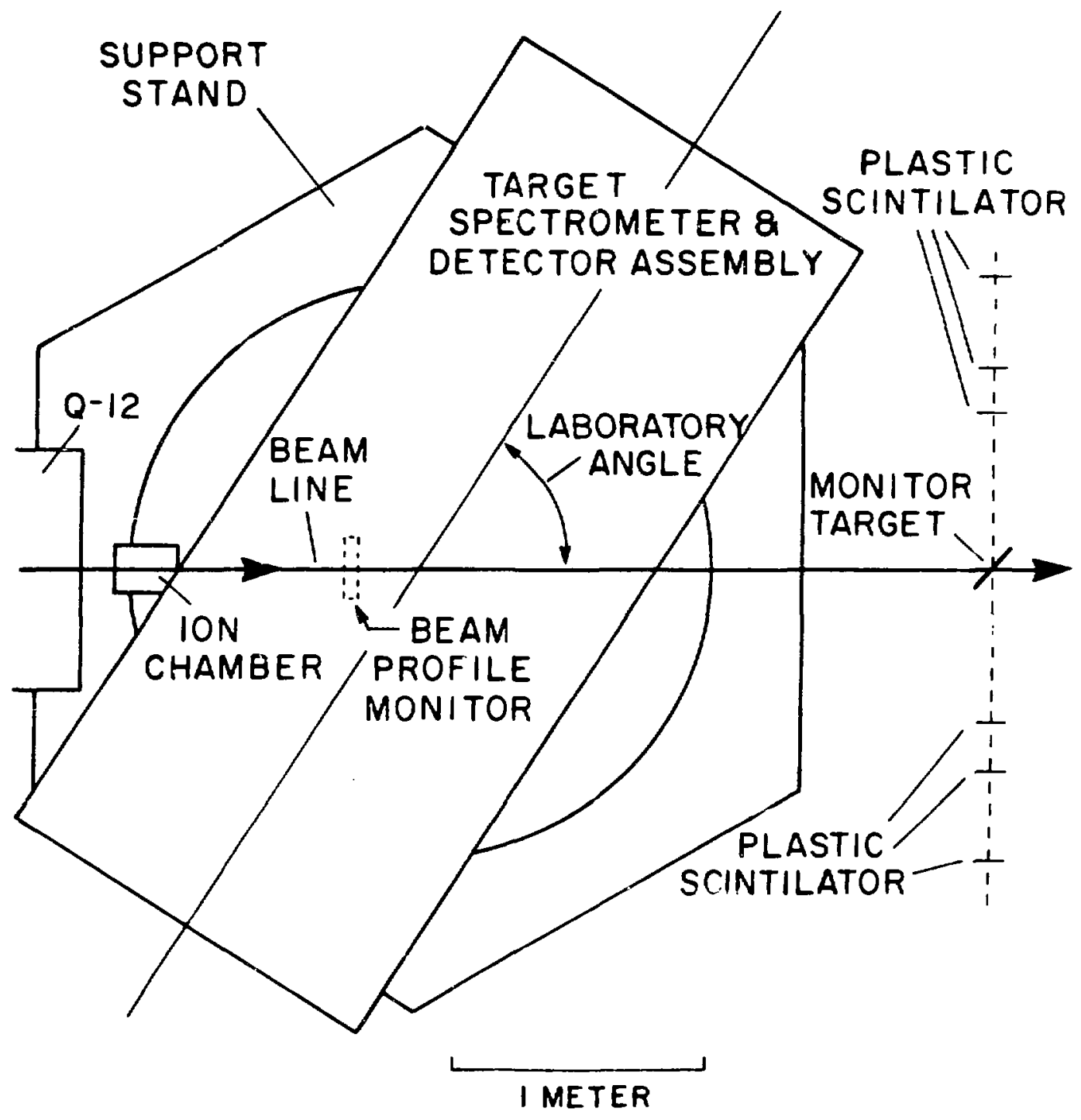

Fig. 1. Plan view of experiment. $Q-12$ is the last quadrapole magnet of $P^{3}$-west. 
the desired beam primarily from the results of a computer simulation, ${ }^{5}$ but some final steering adjustments were made on the basis of the pair of multiwire proportional chambers just upstream of the liquid hydrogen target, indicated as beam profile monitor in Fig. $l$.

The momentum of the beam was one of the inputs to the computer simulation of the channel, and so was presumably known. The kinematics of $\pi^{-p}$ elastic scattering reliably yielded the beam momentum, with a result about $2 \%$ lower than the expected value. This deviation and the final beam adjustments both now appear to be due to an error in the conversion from shunt voltage to magnetic field strength for the first bending magnet of $P^{3}$, which determines the momentum. When $P^{3}$ was tuned according to the simulation, the profile monitor, described below, showed the beam to be off the beam line and to have a washed out profile. The last two of the three $\mathrm{P}^{3}$ bending magnets were alternately twiddled to optimize the profile and bring the beam back on beam line. The beam was brought to a waist at the hydrogen target with a spot size of $1.5 \mathrm{~cm}$ vertical by $1.2 \mathrm{~cm}$ horizontal, full width at half maximum (FWHM), as measured by the profile monitor.

The profile monitor was developed at LAMPF and has been described by Krausse and Gram. ${ }^{\epsilon}$ It consisted of a pair of multiwire proportional chambers which detected the beam profile in both the horizontal and vertical directions. The two planes were separated by $1 \mathrm{~cm}$, and each contained 64 wires with $0.2 \mathrm{~cm}$ spacing between wires. The chambers were filled with a mixture of $80 \%$ argon and $20 \%$ carbon, and were operated at about $-2.4 \mathrm{kV}$. The current from each wire was converted to a voltage, the peak voltage detected and held long enough for the multiplexer to complete the scan of all 64 channels. The output from each chamber was displayed on an oscil8 
loscope, providing a histogram of the voltage for each wire. The multiplexer scan and the oscilloscope were triggered by a beam gate logic pulse which indicated the temporal structure of the beam.

\section{Liquid Hydrogen Target}

The target flask, shown in Fig. 2, was a $2.54 \mathrm{~cm}$ diameter cylinder of $50 \mu \mathrm{m}$ mylar. It was oriented coaxially with the vertical axis of rotation of the spectrometer. ${ }^{7}$ The two ends of the cylinder were epoxied to stainless steel tubing which connected the flask to the cryodyne Model 1022 refrigerator and the reservoir of liquid hydrogen located above the flask as shown in Fig. 3. The refrigerator, reservoir and flask were enclosed in the vacuum system of the spectrometer and rotated with it. The hydrogen system was insulated from radiative heat transfer by super insulation which can be seen on the refrigerator, part of the reservoir, and the target flask in Fig. 3. The gaseous hydrogen which boiled from the liquid hydrogen in the target was returned to the refrigerator through the tube at the top of the flask and a helium operated valve (in series with the tube) when the valve was open. When the valve was closed, gaseous hydrogen collected beneath the valve, forcing the liquid hydrogen from the flask into the reservoir through the tube at the bottom of the flask. The valve provided a means of emptying and filling the target. The $1 \mathrm{k} \Omega$ composition resistors shown in Fig. 2 at the top and bottom of the flask served as level sensors which were read remotely. The slit in the super insulation on either side of the flask in Fig. 3 permitted a visual check on the presence of liquid hydrogen in the flask. 


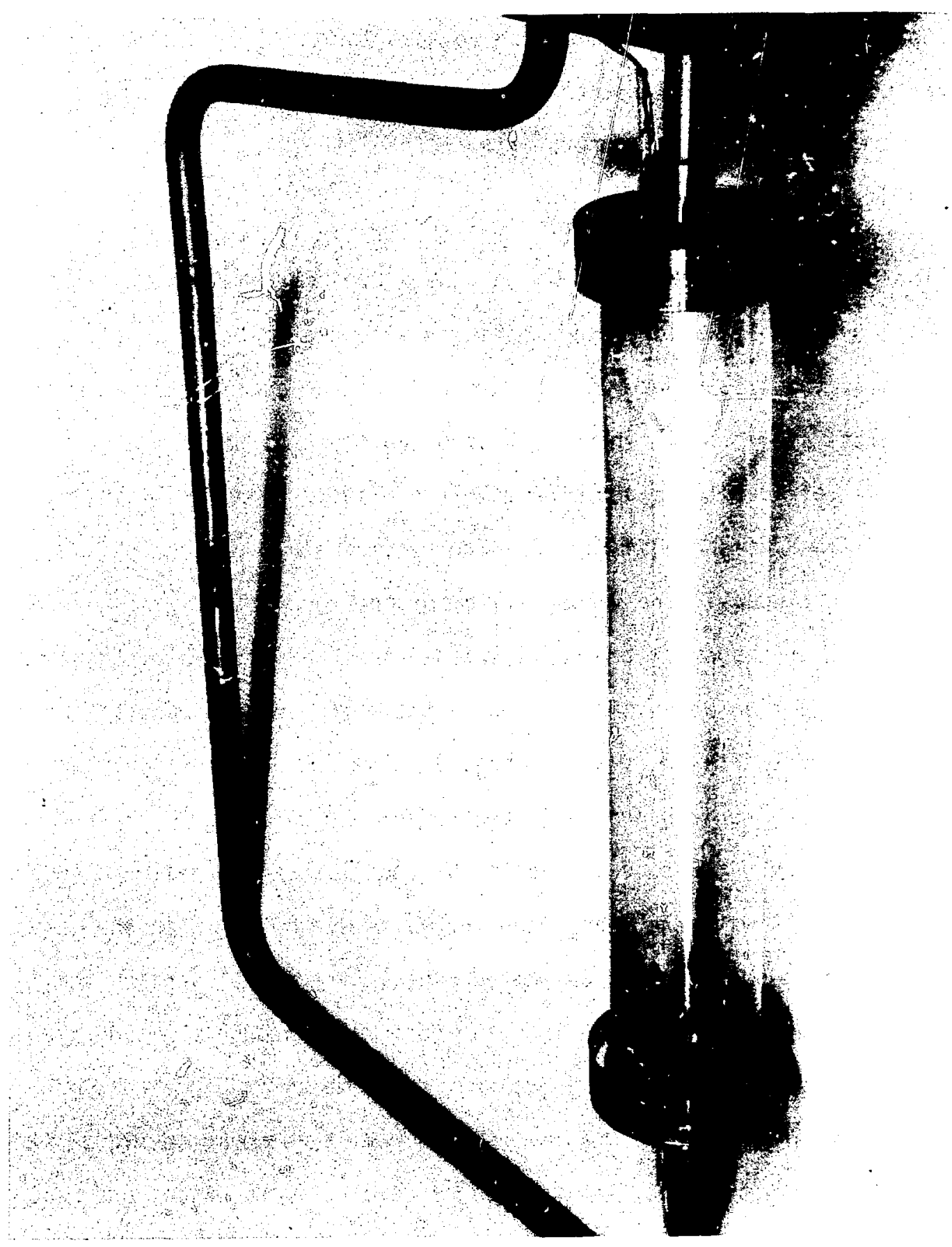

Fig. 2. Liquid hydrogen target flask. 


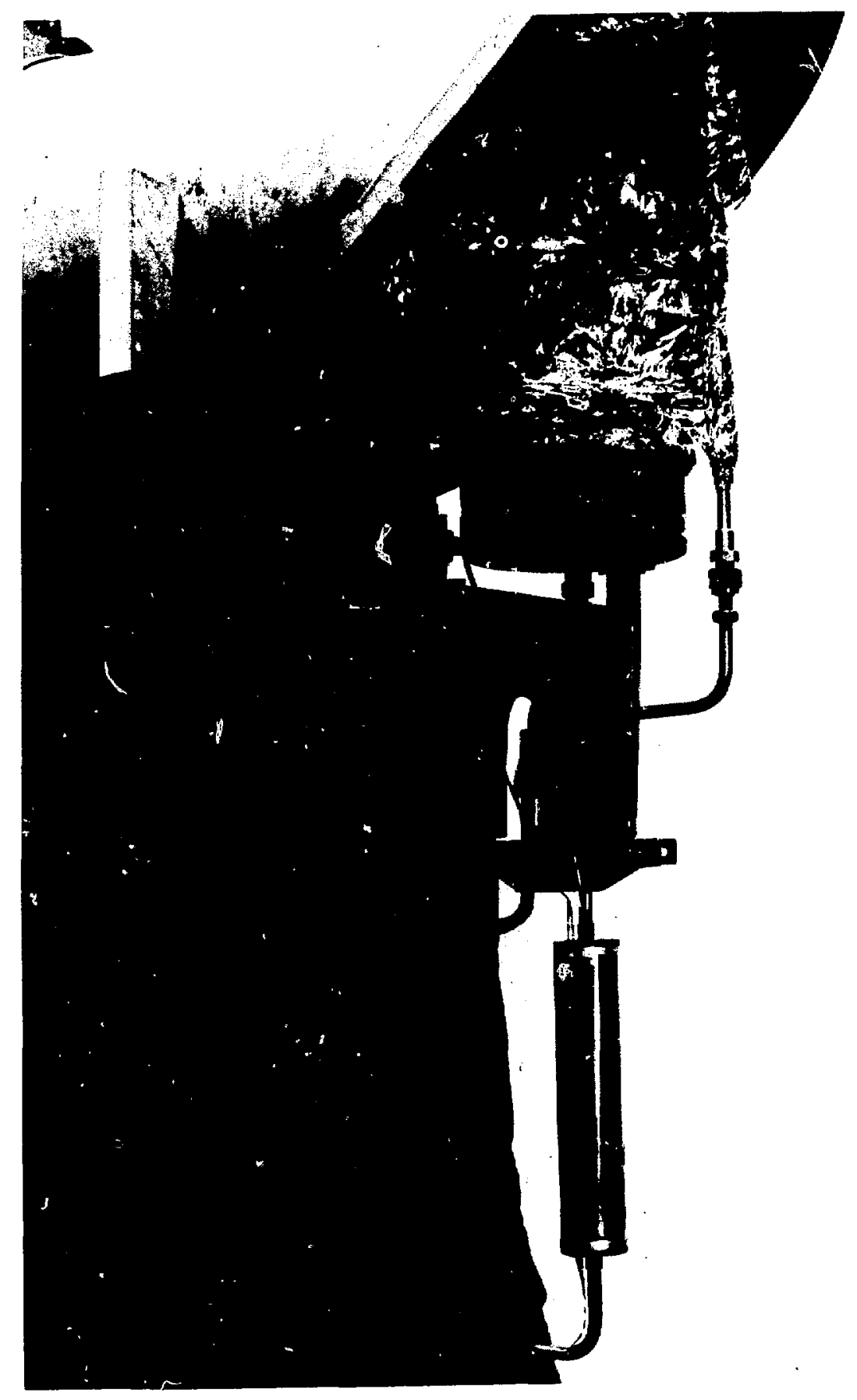

Fig. 3. Target flask with reservoir and refrigerator. 


\section{Spectrometer}

The $180^{\circ}$, vertical bend double focusing, magnetic spectrometer and the detector system are shown in profile in Figs. 4 and 5. Properties of the spectrometer are summarized in Table II. The design and early history of the spectrometer are related by Oyer. ${ }^{8}$ Particles which entered the spectrometer with the selected charge and momentum were bent vericically through $180^{\circ}$ by the two $90^{\circ}$ bending magnets, and focused onto a ladder of nineteen surface barrier detectors which covered a $\Delta p / p$ of 9.5\%. The target, the path through the magnets, and the ladder of detectors were enclosed in a continuous vacuum to minimize loss of energy and multiple scattering of the particles prior to reaching the surface barrier detectors. After passing through the ladder, the particles continued outside the vacuum to three plastic scintillation detectors and a threshold Cerenkov detector.

The entire assembly of the cryostat, liquid hydrogen target, and the spectrometer was mounted on a Navy Surplus 5 inch gun mount, and was conveniently rotated about the vertical axis of the cylindrical target. The vertical configuration of the spectrometer placed the detector system and the spectrometer out of the beam, permitting the assembly to be rotated to laboratory angles between $32^{\circ}$ and $130^{\circ}$. These restrictions resulted principally from the bulk of the assembly and its proximity to the final quadrapole magnet of $P^{3}$ - west, denoted by $Q-12$ in Fig. 1.

The momentum was determined by the uniform magnetic fields of the bending magnets. These fields were related to the shunt voltage, the voltage drop across a metal alloy resistor in series with the two mag- 


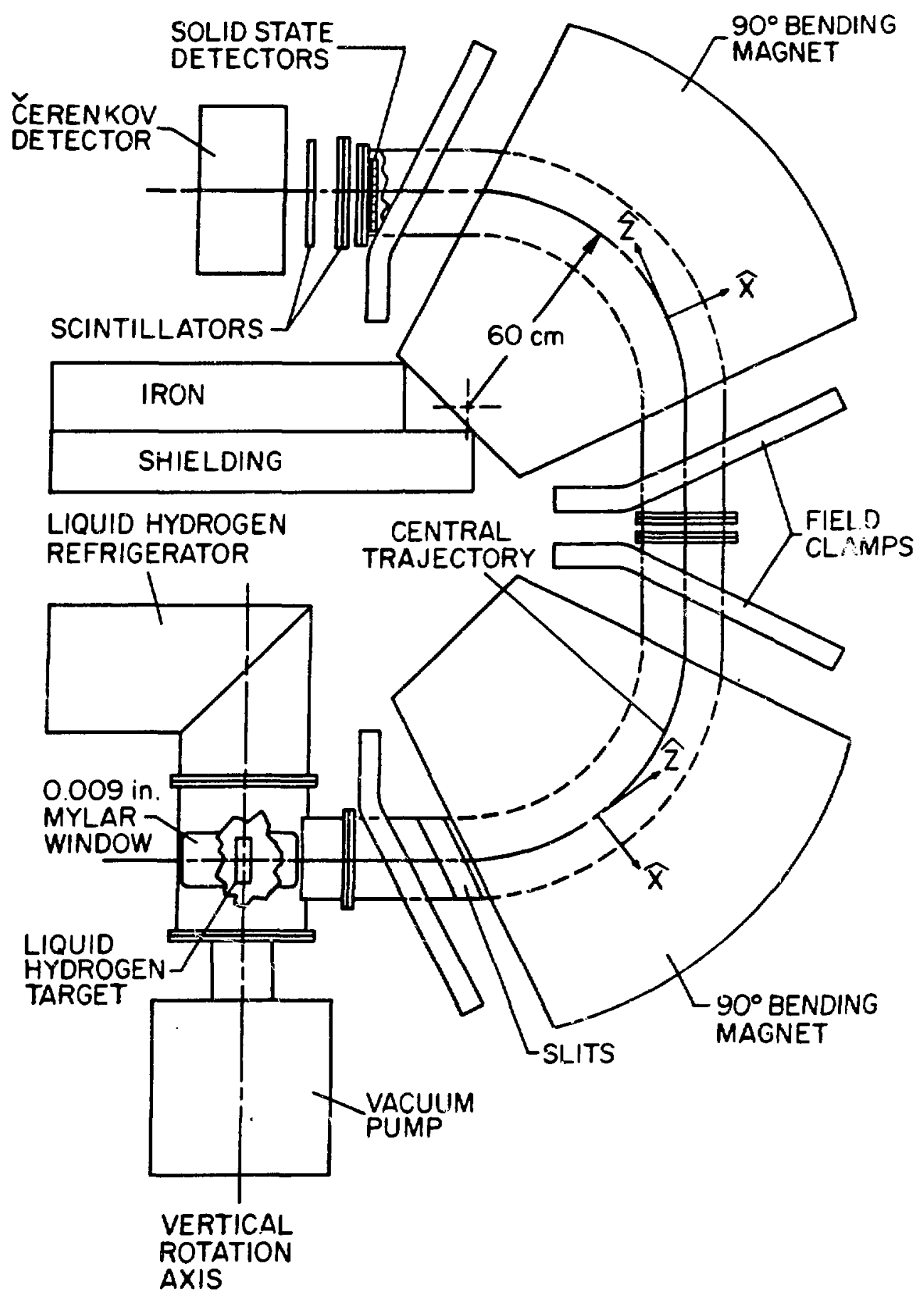

Fig. 4. Profile of spectrometer and detector system. 


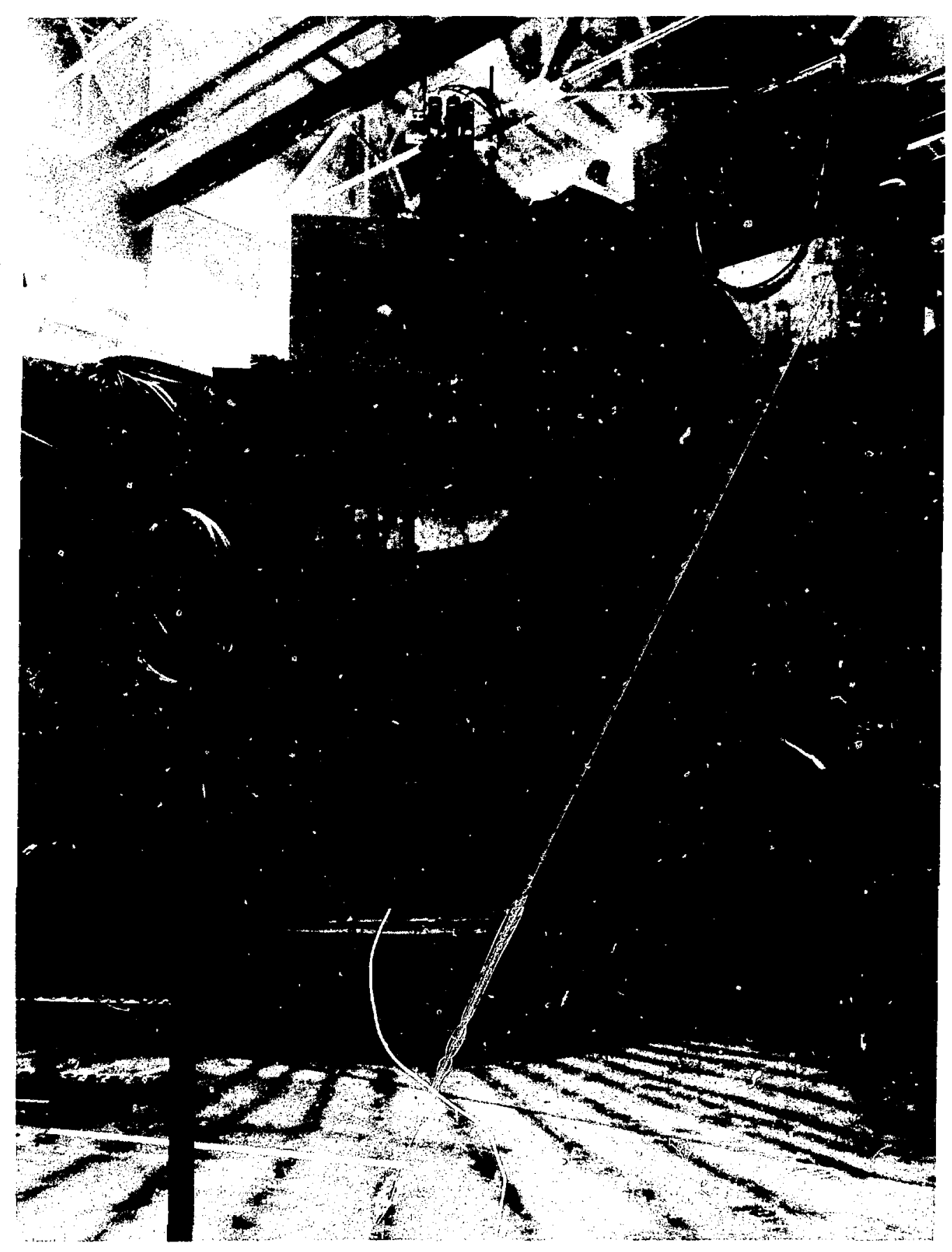

Fig. 5. Profile of spectrometer. 
Table II. Spectrometer properties.

$\begin{array}{ll}\text { Conversion from field strength } & \text { to central momentum, a }=18.119(\mathrm{MeV} / \mathrm{c}) / \mathrm{kG} \\ \text { dispersion } & =0.4351 \pm 0.0004 \% / \mathrm{cm} \\ & =0.4895 \pm 0.0005 \% / \text { channel } \\ \text { magnification } & =-0.96 \\ \text { solid angle acceptance } & =15 \mathrm{msr} \\ \text { scattering angle acceptance } & =2.5^{\circ} \\ \text { upper momentum limit } & =325 \mathrm{MeV} / \mathrm{c}\end{array}$


nets, and were found to be well represented by functions of the form ${ }^{8}$

$$
H(x)=A+B \sigma(1+\exp (-\mu / \sigma)) \ln \{(1+\exp (\mu / \sigma)):(1+\exp ((\mu-x) / \sigma))\}
$$

where $x$ is the shunt voltage. Table III displays the parameter values used for each magnet. The slope of this function, $d H / d x$, is a Fermi function with the shunt voltage analogous to the energy, the parameter $\mu$ analogous to the chemical potential, and the parameter $\sigma$ analogous to $k T$. The field is thus represented as nearly linear for small shunt voltage and rolling off to a constant saturation level for large shunt voltage. The nominal spectrometer momentum was

$$
p=\frac{1}{2}\left\{H_{1}(x)+H_{2}(x)\right\} \text { a } q
$$

where $H_{3}$ and $H_{2}$ were the fields in the two magnets, $x$ the shunt voitage, a was a conversion factor determined experimentally as described in Chapter III, and $q$ was the particle charge in units of the electron charge. A particle entering the spectroneter from the renter of the target, having the nominal spectrometer momentum and the selected charge sign, follcwed a trajectory which passed through the center of the focal plane. A particle with a momentum differing by $\Delta p$ followed a trajectory which was displaced at the focai plane by a distance equal to the product of $\Delta p / p$ with $\delta$ the dispersion of the spectrometer. This displacement was measured by the ladder of surface barrier detectors. Each of these detectors in coincidence with the three scintillation detectors defined a detection channel. Thus there were nineteen detection channels, each corresponding to a different range of displacement from the center of the focal plane.

The solid angle acceptance for a detection channel was in each case 
Table III. Parameter values for spectrometer excitation function.

\begin{tabular}{lcccc}
\hline & A (Gauss) & B (Gauss/mV) & $\mu(\mathrm{mV})$ & $\sigma(\mathrm{mV})$ \\
\hline Magnet 1 & $12.55 \pm 8.98$ & $278.9 \pm 0.42$ & $74.02 \pm 1.13$ & $7.436 \pm 0.54$ \\
Magnet 2 & $19.65 \pm 3.45$ & $277.8 \pm 0.15$ & $72.34 \pm 0.39$ & $6.484 \pm 0.19$ \\
\hline
\end{tabular}


1 imited by the walls of the vacuum chamber and thus varied from channel to channel. The particle transport model of the spectrometer predicted that the solid angie acceptance for the extreme channels would be $40 \%$ less than that for the central channel. This calculation coulc not include an unknown variation in the sensitive areas of the surface barrier detectors and other possible effects, and it was thus essential to measure the variation in the solid angle acceptance, referred to as efficiency. This determination, described in Chapter III, provided the results in Table IV after the efficiencies were normalized to 1.0 for the tenth or central detector. The efficiencies are also shown in Fig. 6, where the statistical uncertainties are aw sut the size of the symbol.

\section{Detectors and Detection System}

The detectors used in this experiment (see Fig. 4) consisted of surface barrier detectors, scintillation detectors and a Cerenkov detector. The nineteen surface barrier detectors were distributed across the focal plane, providing momentum resolution. They also aided in particle identification for momenta below $90 \mathrm{MeV} / \mathrm{c}$ through $\mathrm{dE} / \mathrm{dx}$ information. A coincidence among the three. scintillation detectors and at least one of the surface barrier detectors signaled the computer which then processed the event. The Cerenkov detector identified $\mathrm{e}^{+}$which had been transported by the spectrometer. This section describes these devices and their accompanying electronics.

The surface barrier detectors were of the silicon variety, each nominally $1 \mathrm{~cm} \times 3 \mathrm{~cm} \times 400 \mu \mathrm{m}$. A previous study showed the system to have a large depth of focus. ${ }^{8}$ Hence the detectors were staggered, 
Table IV. Detection channel efficiencies.

\begin{tabular}{|c|c|c|}
\hline Channel & $n$ & $\Delta n(\%)$ \\
\hline 1 & 0.5786 & 0.78 \\
\hline 2 & 0.6701 & 0.75 \\
\hline 3 & 0.7626 & 0.73 \\
\hline 4 & C. .8607 & 0.70 \\
\hline 5 & 0.9039 & 1.00 \\
\hline 6 & 0.9631 & 0.69 \\
\hline 7 & 1.0103 & 0.69 \\
\hline 8 & 0.9787 & 0.71 \\
\hline 9 & 0.9923 & 0.70 \\
\hline 10 & 1.0000 & 0.70 \\
\hline 11 & 0.9509 & 0.72 \\
\hline 12 & 0.9573 & 0.72 \\
\hline 13 & 0.9618 & 1.04 \\
\hline 14 & 0.9494 & 0.71 \\
\hline 15 & 0.8633 & 0.74 \\
\hline 16 & 0.8031 & 0.76 \\
\hline 17 & 0.7379 & 0.79 \\
\hline 18 & 0.6730 & 0.84 \\
\hline 19 & 0.5900 & 1.00 \\
\hline
\end{tabular}




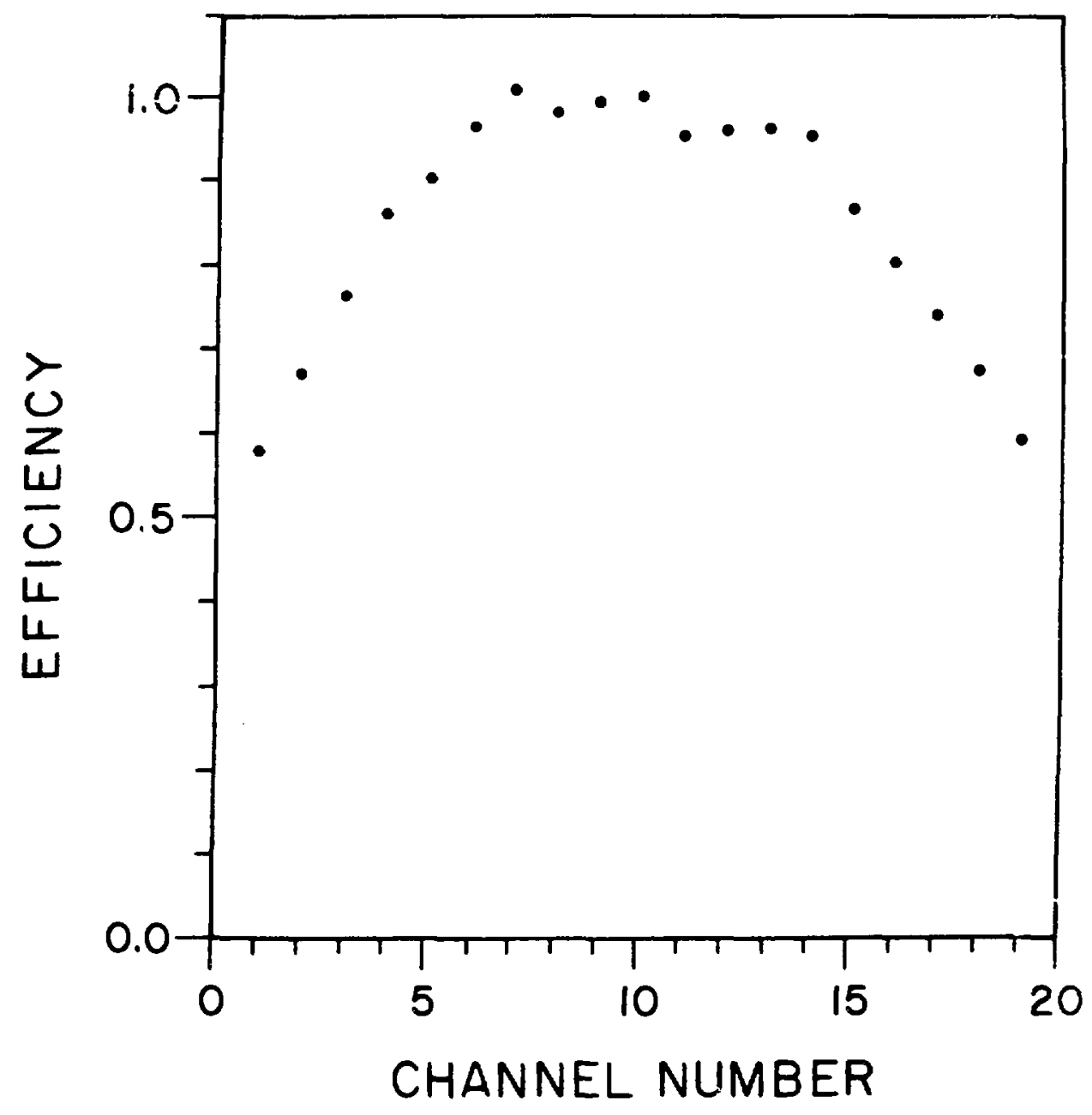

Fig. 6. Detection channel efficiencies. The errors are about the size of the dots. 
positioned alternately $0.2 \mathrm{~cm}$ on either side of the focal plane, to allow them to be located on $1.125 \mathrm{~cm}$ centers as shown in Fig. 7 . The devices were distributed along the dispersion direction and thus provided momentum resolution.

Each of the silicon detectors was reverse biased with sufficient voltage to extend the depletion layer to the full thickness of the device. Thus the entire volume of the detector was sensitive to charged parti.les, providing an optimum electrical efficiency. The signal from the detector was a current pulse with a total charge proportional to the energy deposited by the particle which had crossed the detector. A solid state preamplifier, located near the detector to minimize the capacitance of the connecting cable, integrated the current and produced an exponentially decaying voltage pulse with a time constant of $7 \mu \mathrm{s}$ and an amplitude proportional to the total charge of the initial pulse. The output drove a shaping amplifier which amplified, integrated again to further reduce the presence of the high frequency noise inherent in solid state detectors, and differentiated twice, ali with a 250 ns time constant. The resulting bipolar pulse, with an amplitude proportional to the original energy deposition, was finally processed by a single channel analyzer which produced a 20 ns negative logic pulse provided the amplitude was between an upper and lower limit. The lower limit was set to accept the level of minimum ionizing particles except for momenta below $90 \mathrm{MeV} / \mathrm{c}$, for which the $\mathrm{Pb}$ absorber just before the third scintillation detector had to be removed to detect the $\pi^{+}$mesons. In these cases the lower limit was raised to eliminate a portion of the soft $e^{ \pm}$background. The upper limit was set at the maximum of the de- 


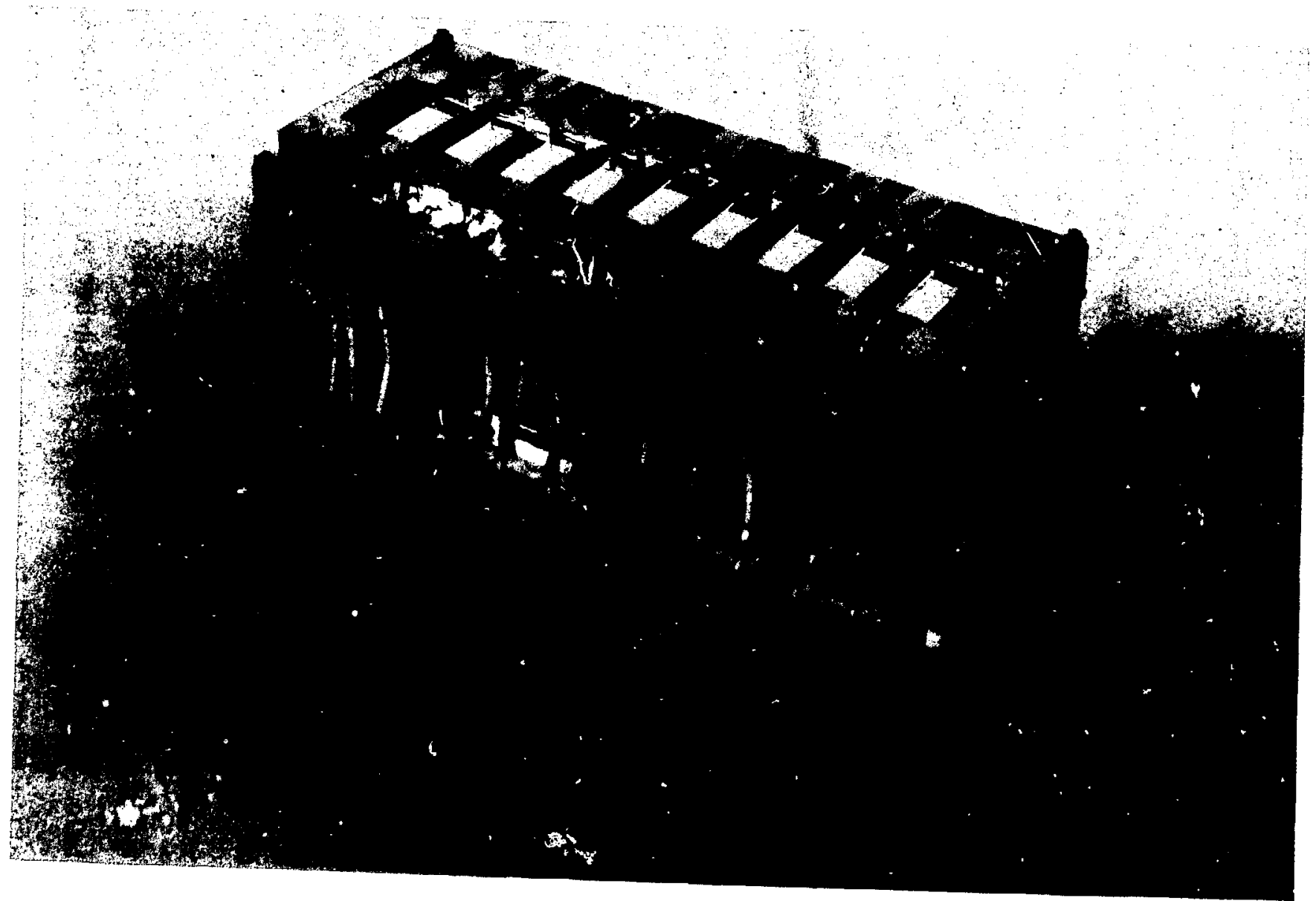

Fig. 7. Ladder of surface barrier detectors. 
vice (protons transported by spectrometer had too little energy to trigger the last scintillation detector). The logic pulse was delivered to an input on one of two CAMAC registers which were read by the computer. The plastic scintillation detectors each measured $26.7 \times 5.7 \times 0.35$ $\mathrm{cm}$ and were located 13,14 and $23 \mathrm{~cm}$ behind the focal plane. A triple coincidence among these detectors gated the surface barrier detectors and the Cerenkov detector. A portion of the background events which were not associated with the spectrometer was thus elminated. $\mathrm{A} 0.8 \mathrm{~mm} \mathrm{~Pb}$ absorber was placed just before the third detector for momenta above $90 \mathrm{MeV} / \mathrm{c}$ to further reduce the soft $\mathrm{e}^{ \pm}$background.

Eachplastic scintillation detector was viewed by an EMI 9815B photomultiplier tube through a Lucite light pipe. The tubes were biased by LAMPF standard bases, and equipped with the accompanying MUMETAL and iron magnetic shields. Each detector drove a discriminator which produced a $20 \mathrm{~ns}$ logic pulse whenever the detector pulse height exceeded a minimum level. These logic pulses were futher processed by the trigger and dead time system.

The Cerenkov detector measured radiation from FC-88 a liquid fluorocarbon marketed by Minnesota Mining and Manufacturing Company. The fluid was contained in a $30 \mathrm{~cm}$ high, $12.7 \mathrm{~cm}$ diameter, $1.6 \mathrm{~mm}$ wal1 aluminum cylinder which was lined with a $0.4 \mathrm{~mm}$ sheet of ALZAK to provide high reflectivity. It wäs viewed by two Amperex 58 DVP photomultiplier tubes, one on either end of the cy?inder. The signals from the photomultiplers were summed in a passive circuit and fed to a discriminator, which produced a logic pulse whenever the combined pulse height exceeded a minimum value. The logic pulse was delayed 110 ns to match the gate pulse and delivered to a CAMAC register. 
The trigger and dead time logic is displayed in Fig. 8. A threefold coincidence among the logic pulses from the plastic scintillation detectors $S_{1}, S_{2}$ and $S_{3}$ produced a gate pulse for the Cerenkov detector. This pulse was also delayed an additional 580 ns by a gate and delay generator and stretched to a width of $200 \mathrm{~ns}$ by a discriminator and used to gate the signals from the surface barrier detectors. The width was necessary to allow for jitter in the 700 ns processing time for the pulses from the surface barrier detectors. Pulses from the surface barrier detectors arriving at the CAMAC registers during the gate pulse were registered. These registers had a summing output which provided a current pulse with an amplitude proportional to the number of inputs which had registered a signal. This signal thus indicated the number of surface barrier detectors producing a pulse in coincidence with the plastic scintillation detectors. The computer was signaled to process the event if at least one of the inputs had received a pulse. The computer subsequently read and cleared the CAMAC registers for the surface barrier detectors and the Cerenkov detector and added the event to the appropriate histcgram. The dead time pulse ensured that no further gate pulse could reach the registers while the event was being processed. The dead time pulse consisted of two parts. A $1 \mu \mathrm{s}$ puise $\tau_{1}$ was put out by a discriminator on the falling edge of $\overline{S_{1} S_{2} S_{3}}$, which allowed sufficient time for the CAMAC registers to receive any pulses from the surface barriers detectors. A pulse from the summing output of the CAMAC registers initiated an extention pulse $\tau_{2}$ fromi a gate generator in addition to notifying the computer. This pulse was terminated by a stop pulse from the computer once it had finished pro- 


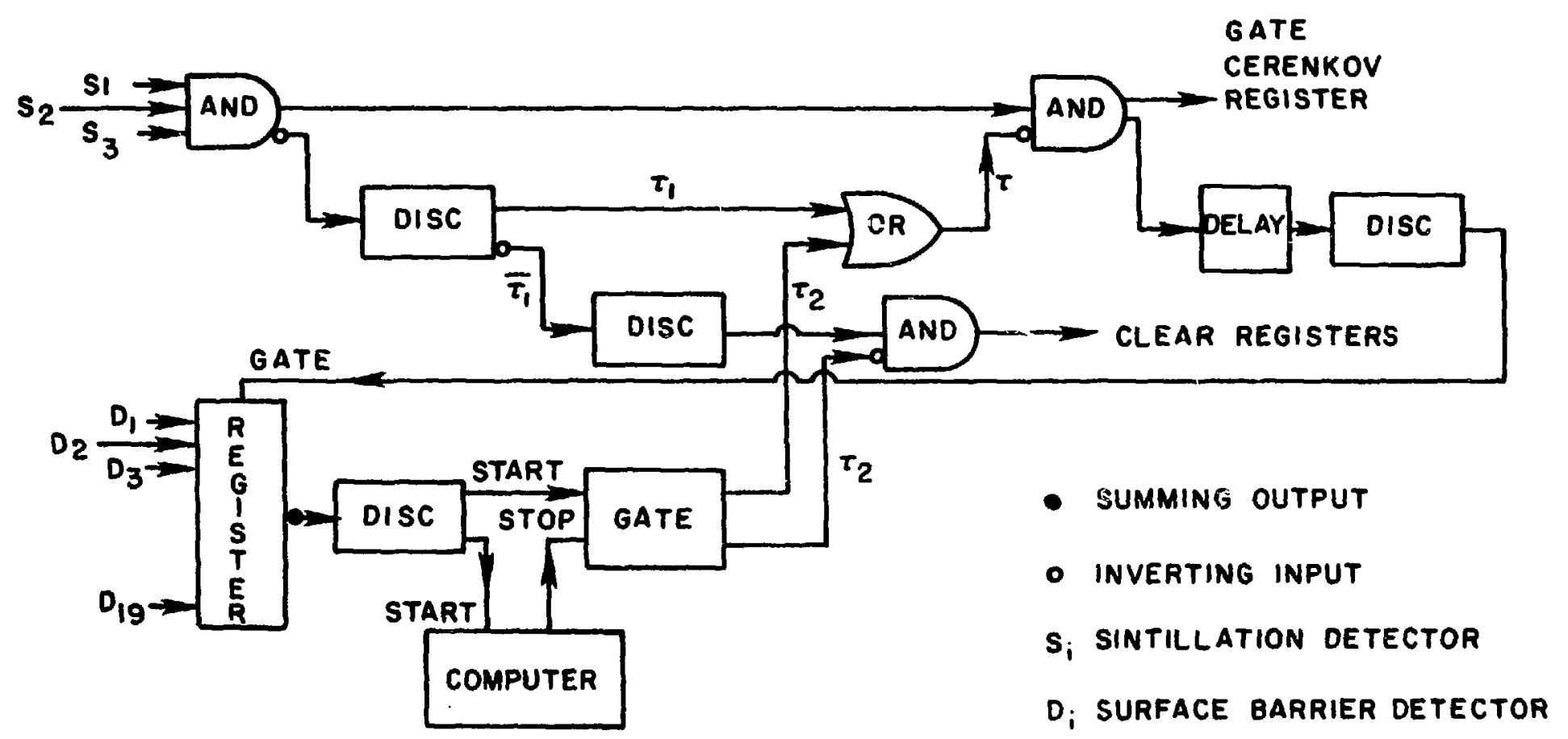

Fig. 8. Trigger and deadtime logic. 
cessing the event. In the instance that no coincident pulse from the surface barrier detectors was registered, the register for the Cerenkov detector might still have received a pulse and had to be cleared. A discriminator put out a clear pulse on the falling edge of $\tau_{1}$. This pulse was vetued by $\tau_{2}$ if it was present, but otherwise cleared the Cerenkov register and the registers for the surface barrier detectors as we11. An estinate of the number of events lost during dead time was possible since various quantities which were scaled, such as $S_{1} S_{2} S_{3}$, were scaled with and without a dead time inhibit as discussed below.

\section{Beam Flux Monitors}

The incident flux of pions was monitored by a scattering monitor and an ion chamber as shown in Fig. 1. The scettering monitor, located downstream of the liquid hydrogen target, was the principal monitor since it was not subject to the vagaries of the ambient temperature and pressure as was the the ion chamber. However, the spectrometer intercepted a portion of the beam for measurements forward of about 400 in the laboratury, and the ion chamber provided the necessary connection with temporally nearby meásurements.

The scattering monitor consisted of a polyethylene target viewed by two counter telescopes as shown in Fig. 1. The polyethylene target measured $23 \times 15 \times 1.3 \mathrm{~cm}$ and was oriented in a vertical plane which cut the beam line and the line of the two telescopes at $45^{\circ}$ angle. Each of the six plastic scintillation detectors measured $15 \times 15 \times 0.3 \mathrm{~cm}$ and was viewed by an RCA 6199 photomultiplier tube through an adiabatic Lucite light pipe. Each telescope required a threefold coincidence 
and primarily counted pions scattered from the beam by the hydrogen and carbon in the polyethylene target. The threefold coincidences were scaled in CAMAC scalers.

The ion chamber consisted of eleven sheets of $6 \mu \mathrm{m}$ doubly aluminized mylar, stretched or aluminum rings at $2.5 \mathrm{~cm}$ intervals along the beam direction. There were four low voltage signal plates interleaved between five high voltage plates while the trin end plates were at ground and served primarily to seal the aluminum can which housed the chamber. The high voltage plates were maintained at 900 volts by a battery power supply. The current from the signal plates was integrated by an ortec 439 current digitizer which put out a pu?se for every $10^{-10}$ Coulombs of charge collected. The signal from the digitizer was scaled in both a visual preset scaler and a CAMAC scaler, with the preset scaler determining the length of the run as dessribed in the next section. Argon was flowed through the chamber at a rate of $0.1 \mathrm{l} / \mathrm{m}$ (replacing the volume about once every 3 hours) and a pressure of $0.3 \mathrm{~cm}$ of octoil above the ambient atmospheric pressure.

\section{Data Acquisition}

The data for this experiment were collected ir. data runs, lasting $\sim 1 / 3$ hour for elastic scattering and between $\sim 1$ hour and $\sim 3$ hours for $\pi^{+}$ production data. For a data run, the target fiask was either empty or full of liquid hydrogen as appropriate, and the spectrometer was set to detect $\pi$ mesons of the desired charge, angle and momentum. The computer was intialized and events were then accumulated during a run gate, which certified that operational requirements were met. After a preset amount of current had been accumulated from the ior, chamber, data acqui- 
sition was halted and the data were punched on paper tape and printed on a teletype. The former was read on another computer which was used for analysis, while the latter was retained for the experiment log.

The computer kept track of four histograms. Each histogram had nineteen bins, the number of counts in a bin indicating the number of events for which the corresponding surface barrier detector had registered a pulse. Events which showed a pulse in only one of the surface barrier detectors and no pulse in the Cerenkov detector were adjed to the first histogram. Events with exactly one pulse in the surface barrier detectors and a puise in the Cerenkov detector were added to the second histogram. The third histogram consisted of events with exactiy two of the surface barrier detectors producing a pulse, whi?e the fourth histogram cons:sted of events with at least three of the surface barrier detectors producirg a pulse. These latter two histograms ignored the Cerenkov detector.

A number of quantities of interest, which are listed in Table $V$, were scaled in CAMAC modules. The digitized signal from the ion chamber, which has a slow response time, was gated only by an intorval gate which indicated that the run had commenced and was in progress. The remaining quantities were each scaled in two scalers, one gated by the run gate described below and one gated by the run gate out inhibited by the dead time pulse described above. The computer periodically read and cleared the scalers ard accordingly adjusted its internal sums. A final read and clear was made when the run was terminated.

The presence of a run gate pulse indicated that four ojerating requirements were sacisfied. First it testif, d that the interval gate 
Table V. Quantities scaled during a run.

\begin{tabular}{|c|c|}
\hline $\mathrm{S}_{1} \mathrm{~S}_{2} \mathrm{~S}_{3}$ & Triple coincidences in the trigger telescope \\
\hline$W_{1} W_{2} W_{3}$ & Triple coincidences in the west arm of the scattering monitor \\
\hline$E_{1} E_{2} E_{3}$ & Triple coincidences in the east arm of the scattering monitor \\
\hline$W_{1}^{\prime} W_{2} W_{3}$ & $\begin{array}{l}\text { Coincidences in the west arm with the signal from the first } \\
\text { scintillator delayed }\end{array}$ \\
\hline$E_{j} E_{2} E_{3}$ & $\begin{array}{l}\text { Coincidences in the east arm with the signal from the first } \\
\text { srintillator delayed }\end{array}$ \\
\hline $\mathrm{t}$ & Pulses from a pulse generator operating at $\sim 10 \mathrm{Mkz}$ \\
\hline $\mathrm{q}_{\mathrm{IC}}$ & Pulses from the current digitizer for the ion chamber \\
\hline
\end{tabular}


described above was present. Second it certified the presence of the beam gate. The primary proton beam at LAMPF had a duty factor of $6 \%$, the protons struck the target which produced $\pi$ mesons for the $p^{3}$ channel in $0.5 \mathrm{~ms}$ clusters at $8.33 \mathrm{~ms}$ intervals. Thus the $\pi$ mesons arrived at the liquid hydrogen target with this same structure. Third it ensured that the computer was not in the process of reading the scalers. Lastly it guaranteed that a particular electronics noise source was absent. Due to a fault in the spectrometer power supply and the electronics for the surface barrier detectors, operation of the spectrometer produced significant spikes in the signals from the surface barrier detectors. These spikes were periodic and may have occurred only during the $94 \%$ of the time that $\pi$ mesons were not striking the target, but rone the less this source of accidentals was explicitly avoided by inhibitirg operation for a period of $8 \mu$ s centered on the spikes. 
CHAPTER III

AUXILIARY EXPERIMENTS AND ANALYSES

Several features of the measurenient apparatus were investigated in auxiliary experiments. The relation between the shunt voltage and the central momentum of the spectrometer was calibrated with alpha particles from the dominant decay mode of ${ }^{241} \mathrm{Am}$. The channel efficiencies were measured by sweeping a spectrum of $\pi^{+}$mesons across each of the surface barrier detectors in turn. The dispersion of the spectometer was also determined through this procedure. A survey of the angular distribution of $\pi^{+} \mathrm{p}$ elastic scattering ai $340 \mathrm{MeV}$ was examined for systematic deviations from the known differential cross section. Lastly, the overall normalization of the measurement system was determined for each $\pi^{-}$ beam utilized in the $\pi^{+}$production study by observing $\pi^{-} p$ elastic scattering with the same apparatus. These measurements also provired the distribution of momenta of the $\pi^{-}$in the incident beam. This chapter presents these procedures and ana?yses.

\section{Calibration of Spectrometer Momentum Curve}

The magnetic field strength in the spectometer magnets had previously been measured as a function of shunt voltage, and had been expressed in the compact form presented in Chapter II. ${ }^{8}$ The proportionality constant between the field strength and the momentum of particles focused on the central detector remained to be determined accurately. An ${ }^{241} \mathrm{Am}$ alpha source, previously prepared for the calibration of the Low Energy Pion (LEP) channel at LAMPF, was well suited to this task. The ${ }^{241} \mathrm{Am}$ 
had been deposited in a $0.1 \times 1.0 \mathrm{~cm}$ rectangle on ar aluminum disk, and could be positioned vertically to simulate the distribution of a $\pi$ beam or horizontally to provide contrast. The spectrum of ${ }^{241} \mathrm{Am}$ includes alpha particles at $5443 \mathrm{keV}(13 \%)$ and $5386 \mathrm{keV}(2 \%)$ as well as at $5485.74 \pm 0.12 \mathrm{keV}(85 \%) .^{9}$ The alpha particles were detected by the surface barrier detectors described in Chapter II, and the unwanted secondary lines were eliminated on the basis of pulse height. The source thus effectively provided monoenergetic alpha particles. The $1 \mu \mathrm{Ci}$ strength was adequate, providing on the order of 150 counts per second when the discriminator was adjusted to ensure that only alpha particles from the $5486 \mathrm{keV}$ line were being counted.

The alpha source was mounted in place of the cryogenic target at beam elevation with the aid of a surveyor's transit. The alpha particles were detected by either of two of the nineteen surface barrier detectors at the focal plane. The pulse from the detector amplifier chain was discriminated on the basis of pulse height and counted in a scaler which was read visually. The discrimination level was selected conservatively with the aid of a multichannel analyzer. The scaler was controlled by another scaler which halted the counting once a preset amount of time had elapsed.

The spectrometer was initiallized in the normal fashion; the current was brought to saturation, lowered to zero, brought to saturation a second time and lowered to the desired value. At this setting the alpha particles were focused just off the detector on the lower momentum side. Pulses from the detector were scaled for $20 \mathrm{sec}$, and the shint voltage, indicating the current, and the number of counts were noted in the log. The current was reduced a sma11, constant amount and the procedure 
iterated until the alpha particles had crossed the detector and were focused on the higher momentum side.

Spectra were acquired in this fashion for both the central detector (\#10) and an adjacent detector (\#9) with the source extended both vertically and horizontally. The spectrum for detector \#10 with the source horizontal, shown in Fig. 9, is representative. This spectrum was remeasured, as a check on reproducibility, after the current had been lowered to zero, reversed (as if to detect negative particles), brought to saturation, lowered to zero, and again reversed (to detect positive particles).

The momentum of the alpha particles was associated with the centroid of the spatial distribution at the focal plane. In chis way the momentum associated with a detector corresponded to the mean momentum of the particles detected provided the momentum distribution varied slowly. Table VI presents the determination of the proportionality constant based on eacin of the spectra. For detector $\# g$ the dispersion determined in the next section is assumed. The uncertainties quoted for the central momentum reflect the uncertainty in the position of both the source and detector as well as in the momentum of the alpha particles. ihe uncertainty in the magnetic field strength is due principally to repeatability, but also includes the uncertainty in determining the centroid of the distribution. The results for the two detectors agree to within the expected $0.1 \%$, while the uncanny agreement for the two orientations of the source must be considered a curiosity. The difference between the last two results for detector \#10 is ascribed to the reproducibility of the field strength, as neither the source nor the detector had been disturbed, and 


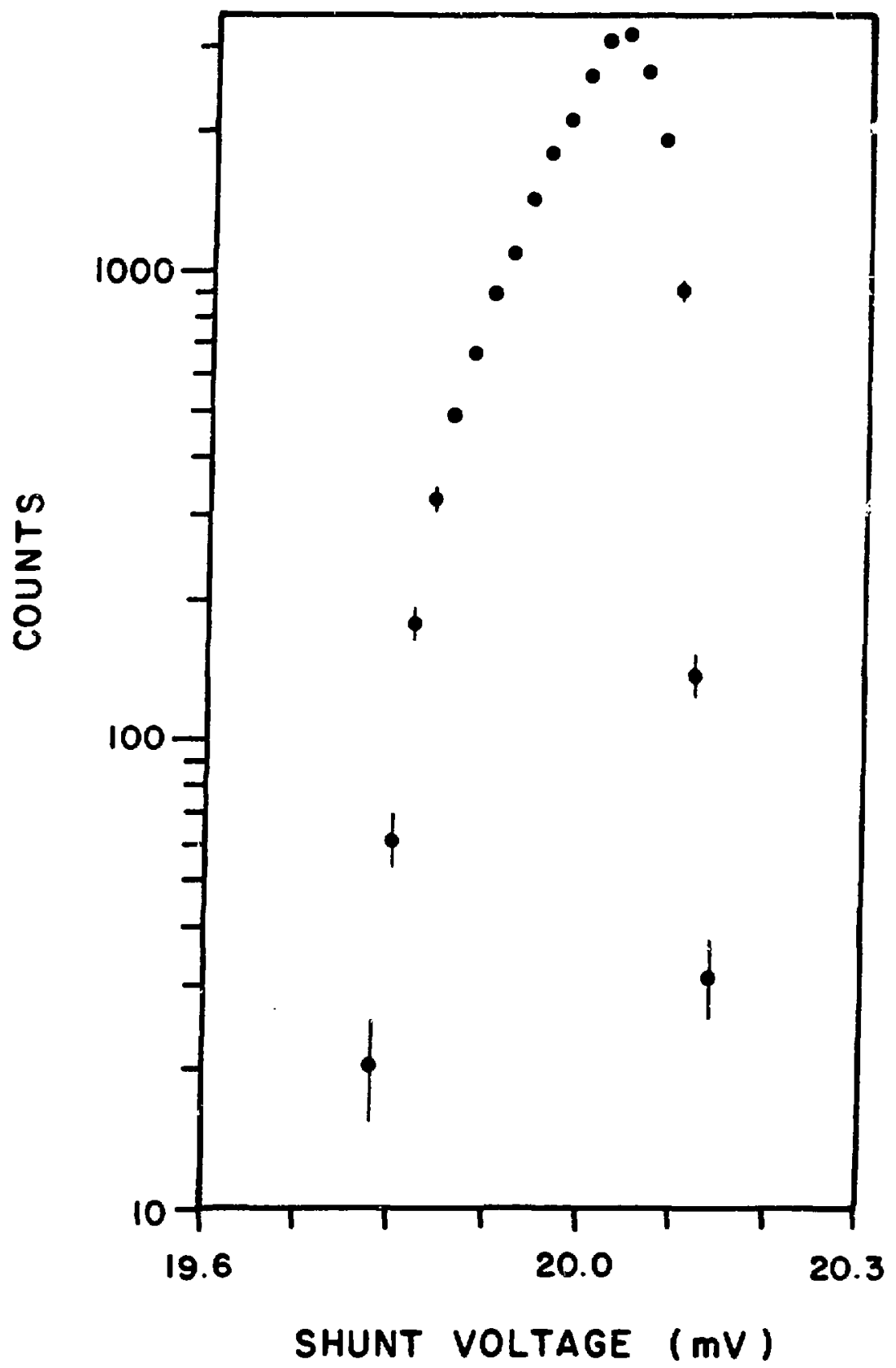

Fig. 9. Sample ${ }^{241} \mathrm{Am}$ spectrum for source extended horizontally. 
Table VI. Results of ${ }^{241} \mathrm{Am}$ data. $i$ is the detector number, $V_{S}$ is the shunt voltage in $\mathrm{mV}, \mathrm{H}$ is the average field strength for the two magnets in $k G, P_{C}$ is the spectrometer momentum in MeV/c, and $a=P_{c} / H$ is in MeV/kGc.

\begin{tabular}{|c|c|c|c|c|}
\hline $\mathbf{i}$ & $v_{s}$ & $H$ & $P_{c}$ & a \\
\hline & \multicolumn{4}{|c|}{ (a) Source extended verticaliy } \\
\hline 9 & 19.923 & $5.561 \pm 0.003$ & $100.66 \pm 0.04$ & $18.101 \pm 0.012$ \\
\hline \multirow[t]{2}{*}{10} & 20.003 & $5.583 \pm 0.003$ & $101.15 \pm 0.04$ & $18.117 \pm 0.012$ \\
\hline & \multicolumn{4}{|c|}{ (b) Source extended horizontally } \\
\hline 9 & 19.921 & $5.560 \pm 0.003$ & $100.66 \pm 0.04$ & $18.102 \pm 0.012$ \\
\hline 10 & 20.000 & $5.582 \pm 0.003$ & $101.15 \pm 0.04$ & $18.120 \pm 0.012$ \\
\hline 10 & 20.014 & $5.586 \pm 0.003$ & $101.15 \pm 0.04$ & $18.107 \pm 0.012$ \\
\hline
\end{tabular}


is of the order expected. The result quoted in Table II represents the average of these values.

\section{Detection Channel Efficiencies}

The measurement efficiency varied from channel to channel due primarily to geometricaldifferences, such as the solid angle acceptance of the channel and the fraction oi the interaction region viewed by the channel, but also due to variations in the sensitive area of the surface barrier detectors. The efficiencies were therefore measured by marching a spectrum across the focal $\mathrm{plane.} \mathrm{In} \mathrm{this} \mathrm{way} \mathrm{the} \mathrm{spectrum} \mathrm{was} \mathrm{regis-}$ tered in each of the channels in turn, with the number of counts in a channel proportional to its efficiency. The efficiencies were determined within an unimportant overall constant. Also, the centroids of the spectra provided an accurate determination of the dispersion of the spectrometer.

The efficiencies were measured using a $140 \mathrm{MeV} \pi^{+}$beam with $\Delta \mathrm{p} / \mathrm{p}$ of $3.8 \%$ (FWHM). The spectrometer was set to detect positive particles scattered from the liquid hydrogen target at a laboratory angle of $50^{\circ}$. For the initial shunt voltage nearly all of the $\pi^{*}$ mesons elastically scattered from the hydrogen had insufficient momentum to be passed by the spectrometer. Events were accumulated until a preset amount of charge had been collected from the ion chamber, at which time the computer output the data. The shunt voltage was decreased a small amount and the procedure repeated until the elastic peak had passed across each of the channels in turn and once again only a small fraction of the elastically scattered $\pi^{+}$mesons were able to pass through the spectrometer.

The raw data was thus divided into 48 runs, one for each value of the shunt voltage. The data for each run consisted of the usual four 
histograms and the accompanying scaler totals. The histogram of the events for which only one of the channels detected a particle, and the number of counts in the scattering monitor during live time for the computer were of interest. The number of counts in each bin was divided by the number of live time counts in the scattering monitor and also by the central momentum (calculated from the shunt voltage). The former normalized each run to the same number of $\pi^{+}$mesons incident on the target during live time, while the latter accounted for the variation in momentum acceptance with central momentum. Finally the bin values were rescaled by a convenient number and resorted according to channel number providing a spectrum for each of the channels.

Each spectrum was fitted to a skewed Gaussian peak plus a constant and an error function term

$$
\begin{aligned}
f(x)= & \zeta_{1} \zeta_{2}^{-1}(2 \pi)^{-\frac{1}{2}} \exp \left(-z^{2} / 2\right)\left\{1-\zeta_{4} z\left(1-z^{2} / 3\right) / 2\right\} \\
& +\zeta_{5}+\zeta_{6}(2 \pi)^{-\frac{1}{2}} \zeta_{z}^{\infty} \exp \left(-y^{2} / 2\right) d y
\end{aligned}
$$

with

$$
z=\left(x-\zeta_{3}\right) / \zeta_{2} \text {. }
$$

The parameters $\zeta_{j}$ were optimized by the nonlinear least squares algorithm described in Appendix A. Some sample spectra with the fitted curves are shown in Fig. 10. This form was justified by a degree of success. The spectra for the three channels which detect particles of the highest momenta contained too few points on the low momentum side of the peak. Consequentially $\zeta_{6}$ could not be determined by the least squares algorithrn, and necessarily was assigned a value. The results of the analysis were relatively insensitive to the particular value assigned.

The efficiencies were proportional to the areas under the peak, 

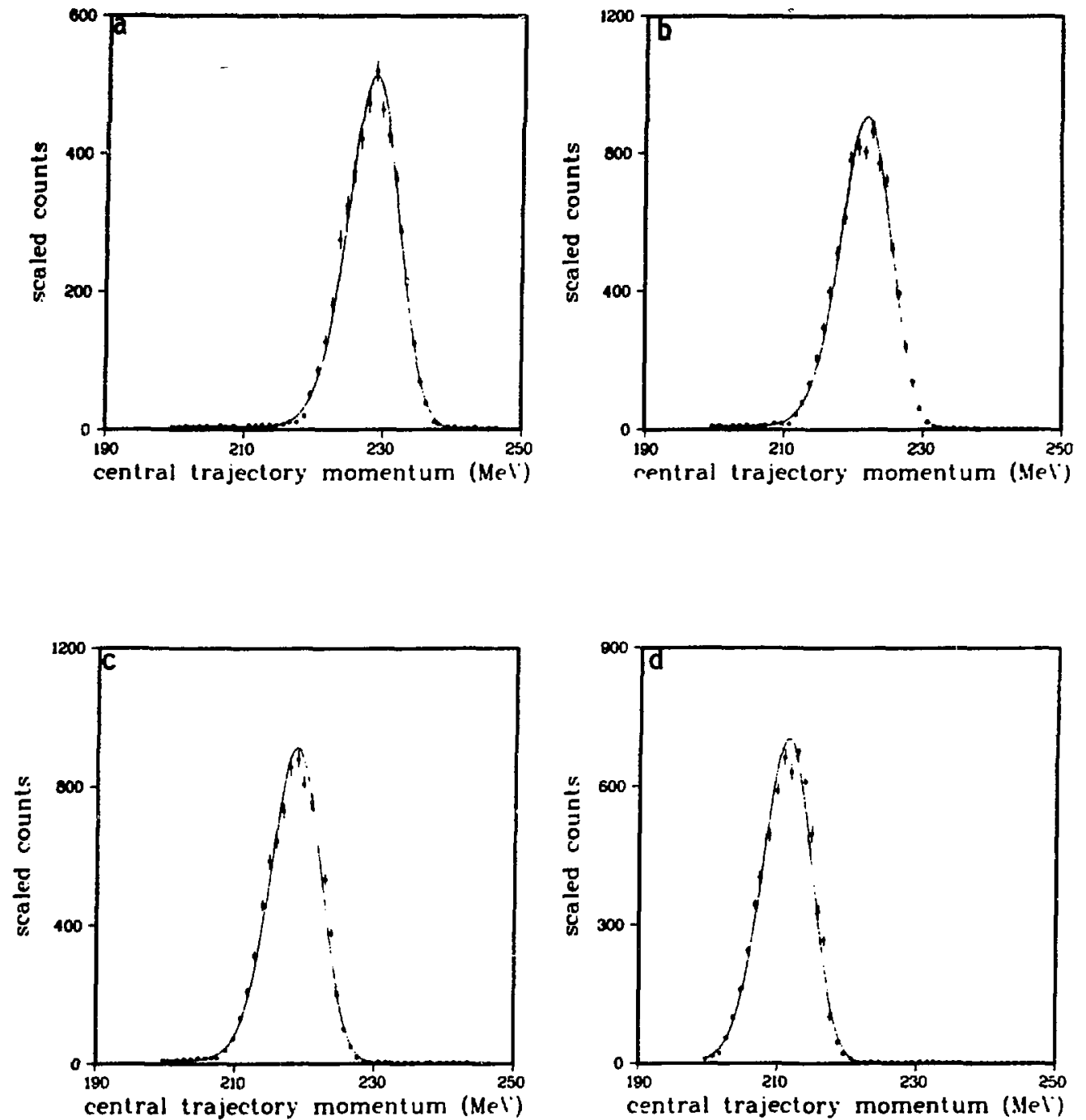

Fig. 10. Sample spectra for channel efficiencies. The curve was detemined by the least squares fitting procedure. 
hence the values of $\zeta_{1}$. The relative efficiencies were defined as

$$
\eta_{i}=\zeta_{1, i} / \zeta_{1,10}
$$

with uncertainties

$$
\Delta n=\left(\Delta \zeta_{1, i}\right) / \zeta_{1,10}
$$

where $i$ indicates the channel number and $\Delta \zeta_{1, j}$ are the uncertainties in $\zeta_{1, i}$ provided by the algorithm. This set of data provided a relative efficiency for each of the detectors, with a statistical uncertainty of about $1 \%$. Although this determination appeared to be completely adequate, the study was repeated as a test of reproducibility. The second study was the same in every respect except that the momentum width of the beam was narrower, $\Delta p / p=2.1 \%$, and hence so was the spectrum of scattered $\pi^{+}$mesons. This resulted in a set $n_{1}$ of seventeen relative efficiencies since two of the surface barrier detectors were malfunctioning. The two sets were compared by defining a $x^{2}$,

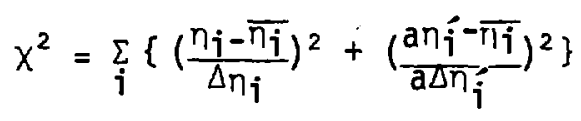

where the sum is over the operative channels, and minimizing with respect to $a$ and the $\overline{\pi_{j}}$. The scale factor allows for the unknown difference in normalization for the two sets of relative efficiencies. The $\overline{\eta_{\mathfrak{j}}}$ are then

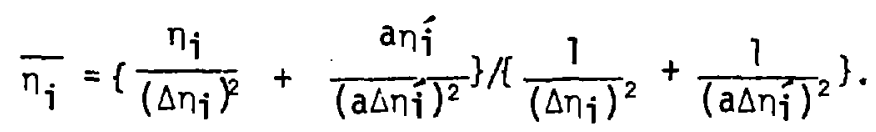

Thn uncertainties were obtained in the same manner as discussed in Appendix $A$. These $\overline{\eta_{i}}$ and their relative, statistical uncertainties were renormalized to obtain $\pi_{10}=-1$, and are presented in Table IV. The 
mininum chi-square was 38 with 16 degrees of freedom, indicating that the iwo sets of relative efficiencies possessed different shapes. The difficulty was associated with the three channels for which $\zeta_{5}$ was necessarily fixed in both studies. As there were no apparent grounds for preferring either determination over the other, the merged values were taken as the best estimates. Aside from these three channels, the two studies were in good agreement showing that the measurements were stable and reproducible.

The spectrimeter dispersion was also determined from these data. The centroids $\zeta_{3}$ of the peak in the spectra represent the values of the central momentum for which the elastic peak was successively centered on each of the surface barrier detectors. In the iowest order approximation, wherein the dispersion $\delta$ is taken to be constant, the spectrum centroids are related to the channel number $i$ and the centroid of the detected momentum distribution $P$ by

$$
y_{i}=P /\{1+(i-10) \delta\} \text {. }
$$

The $\zeta_{3, i}$ with their uncertainties $\Delta \tau_{3, j}$, from the analyses of the spectra, were fitted to this form treating both $P$ and $\delta$ as free parameters. The fitted curve and the points for the first study are shown Fig. 11. The constant dispersion approximation does remarkably well. The $x^{2} / v$ was 2.9, in part due to a $0.5 \mathrm{~mm}$ uncertainty in the positions of the detectors (which was ignored). The dispersion was determined to be $0.4893 \pm$ $0.0008 \%$ per channel from the first siudy and $0.4896 \pm 0.0006 \%$ per channel from the second study. The dispersion was assigned the mean value of $0.4895 \%$ per channel or $0.4351 \%$ per $\mathrm{cm}$ with a statistical uncertainty of one part in $10^{3}$. 


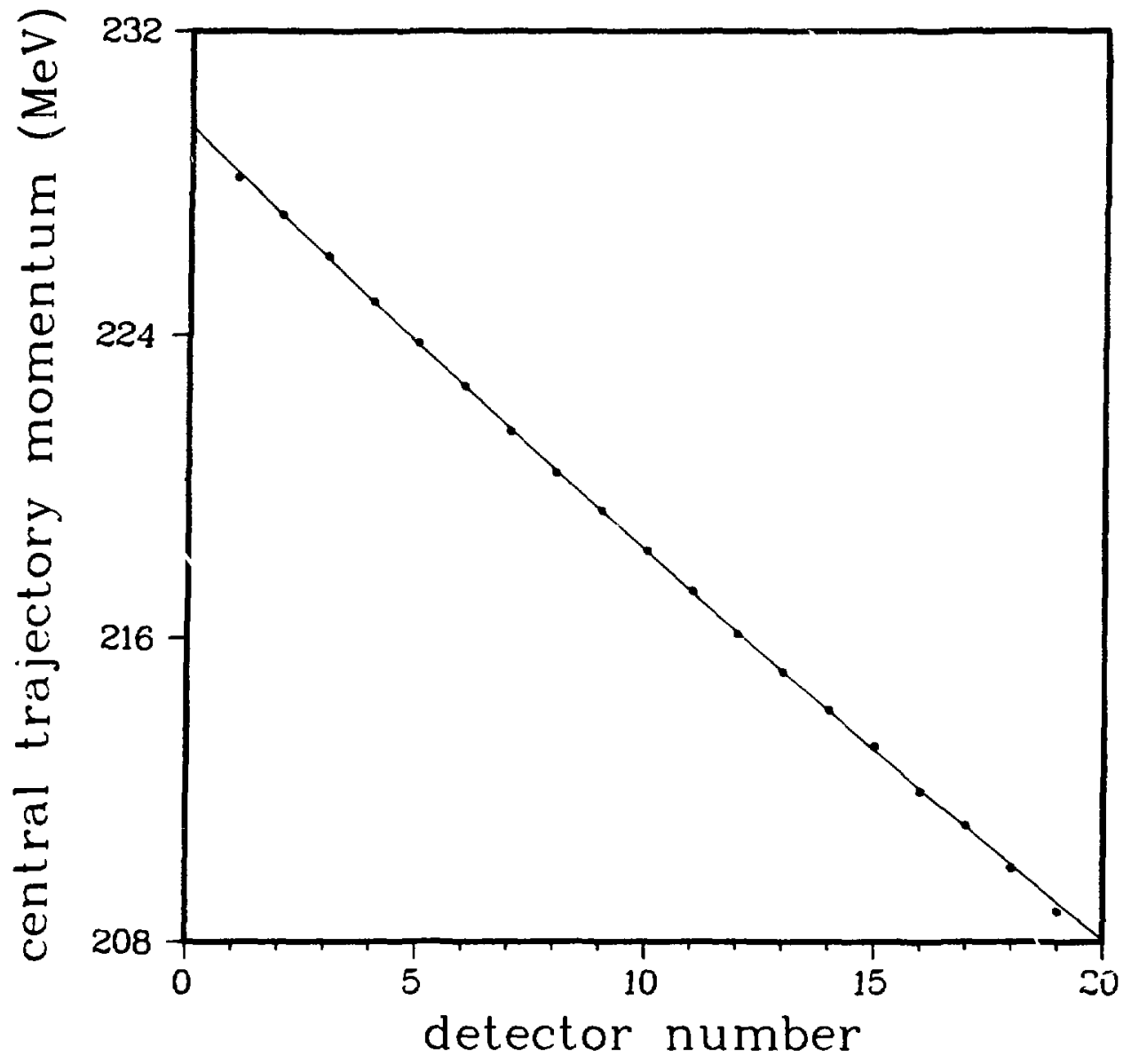

Fig. 11. Data for spectrometer dispersion. The curve was determined by the least squares fitting procedure. 
Angular Survey of $\pi^{+} p$ Elastic Scattering

The solid angle acceptance of the spectrometer was expected to vary slightly with laboratory angle because the interaction region was not cylindrically symmetric. Therefore, a study of $\pi^{t} p$ elastic scattering at $140 \mathrm{MeV}$ was carried out, and the resulting angular distribution was examined for systematic deviation from the prediction of SCATPI, 10 a subroutine based on a phase shift analysis (described in Chapter IV). The predictions are quite successful in this region, allowing a comparison on the $1 \%$ level. Furthermore, the momenta of the scattered $\pi^{+}$mesons were low enough to allow measurement as far forward as $35^{\circ}$ in the laboratory. The analysis of the elastic spectra also provided the momentum distribuition of the incident $\pi^{+}$beam. Thus this study additionally provides a basis for evaluating the consistency or these results.

The investigation was made with a beam of $140 \mathrm{MeV} \pi^{+}$mesons having a $\Delta \mathrm{p} / \mathrm{p}$ of $2.1 \%$ (FWHM). The $\pi^{+}$mesons elastically scattered from protons were detected by the spectrometer for each of twelve angles from $35^{\circ}$ to $130^{\circ}$ in the laboratory. The data for an angle were converted into a spectrum, and the spectrum analyzed to determine the area under the elastic peak. The areas.were corrected for $\pi^{+}$decay and renormalized to agree on the average with the predicted $\pi^{+} p$ differential cross section. The remaining discrepancies were scrutinized for a systematic pattern. The analysis of the spectrum revealed the momentum distribution of the beam through the two body kinematics. The results for the twelve spectra riere examined for consistency.

The data for an angle consisted of the histogram of events for which only one of the channels had detected a particle, the sums of the 
scaler events, the laboratory angle, and the spectrometer shunt voltage. The shunt voltage determined the central momentum $P_{c}$ and hence the momenta $p_{j}$ of the particles detected in each of the channels

$$
p_{i}=P_{c}\{1+(i-10) \delta\},
$$

with $i$ the channel number and $\delta$ the spectrometer dispersion in percent per channel. The number of counts $n_{j}$ in each channel was corrected for the channel efficiency $\eta_{j}$ and the momentum acceptance $P_{c} \delta$, and normalized by the number of counts $M_{\ell}$ in the scattering monitor during live time,

$$
y_{i}=n_{i} /\left(M_{\ell} \eta_{j} P_{c} \delta\right) \text {. }
$$

The spectrometer blocked a portion of the beam from reaching the scattering monitor for laboratory angles forward of about $40^{\circ}$, hence for the measurement at $35^{\circ} M_{\ell}$ above was replaced with $M_{\ell}^{\prime}$, the corrected sum of the live time counts in the monitor

$$
M_{\ell}^{\prime}=\left\langle M_{T} / q_{I C}>q_{I C} M_{\ell} / M_{T} .\right.
$$

Here $M_{T}$ was the number of counts in the scattering monitor ignoring the dead time inhibit, $q_{I C}$ was the charge collected from the ion chamber, and the average was over the values from the other eleven angles. The uncertainty in $y_{i}$ was

$$
\Delta y_{i}=y_{i}\left\{\left(\Delta n_{j} / n_{j}\right)^{2}+\left(n_{i}+1\right) / n_{i}{ }^{2}\right\}^{\frac{1}{2}}
$$

where, since $M_{\ell}, P_{c}$ and $\delta$ were the same for each channel, their uncertainties were not included.

The $y_{j}$ were fitted to much the same function as was employed in 
the previous section

$$
\begin{aligned}
f\left(p_{i}\right)= & \zeta_{1} \zeta_{2}^{-3}(2 \pi)^{-\frac{1}{2}} \exp \left(-z^{2} / 2 \times 1-\zeta_{4} z\left(1-z^{2} / 3\right) / 2\right\} \\
& +\zeta_{5}+\zeta_{6}(2 \pi)^{-\frac{1}{2}} \int_{z}^{\infty} \exp \left(-x^{2} / 2\right) d x
\end{aligned}
$$

with

$$
z=\left(p_{i}-\mu\right) / \sigma \text {. }
$$

Here, however, the centroid $\mu$ and the width $\sigma$ of the detected momentum distribution were parameterized in terms of the momentum distribution of the incident $\pi^{+}$beam. The centroid was computed as

$$
\mu=p-p_{\text {loss }}-p_{\text {ang }}
$$

where $p$ is the momenum of the scattered $\pi$ meson calculated from kinematics from $\tau_{3}$, the mean momentum of the incident beam, $p_{\text {loss }}$ is a correction for momentum lost to atomic electrons in the target and $\mathrm{P}_{\mathrm{ang}}$ is a correction due to the difference between the mean scattering angle and the nominal scattering angle resulting from the finite angular acceptance of the spectromater and emittance of the pion channel. $p_{10 s s}$ and $p_{\text {ang }}$ depend on $\zeta_{3}$, and are presented in Appendix $B$. The width was calculated as

$$
\sigma=\left\{\left(\frac{d p}{d \zeta_{3}} \zeta_{2}\right)^{2}+\sigma_{\text {spot }}^{2}+\sigma_{\text {ang }}^{2}+\sigma_{\text {loss }}^{2}+\sigma_{\text {coul }}^{2}+\sigma_{\text {strag }}^{2}\right\}^{\frac{3}{2}}
$$

The first term represerits the contribution from the momentum spread of the incident beam, while $\sigma_{\text {spot }}^{2}$ represents the contribution arising from the extended image of the interaction region at the focal plane. $\sigma_{\text {spot }}$ is easily calculated from the vertical extent of the beam, the magnification of the spectrometer, and the dispersion of the spectrometer. Briefly, $\sigma_{\text {ang }}^{2}$ is the contribution from the mean square variation in the cosine of the scattering angle due to the finite angular emittance of 
the channel and acceptance of the spectrometer, $\sigma_{\text {loss }}^{2}$ is due to the mean square variation in the momentum lost in the target through variation in the path length in the target, $\sigma_{\text {coul }}^{2}$ is due to the mean square. variation in the scattering angle which results from the small angle Coulomb scattering, and $\sigma_{\text {strag }}^{2}$ is due to the mean square variation in the momentum lost to atomic electrons through the stochastic nature of collisions. These four contributions all depend on the beam momentum $\zeta_{3}$ and each is presented in Appendix $B$. The quadrature summation of these terms is justified only by a degree of success.

Thus the least squares analysis at each angle determined the area under the skewed Guassian peak, and also che centroid $\zeta_{3}$ and width $\zeta_{2}$ of the momentum distribution of the incident beam. Some examples of the spectra and fitted curves are shown in Fig. 12. Each area was converted to a center of momentum cross section

$$
\frac{d \sigma}{d \Omega}=\zeta_{1} E J T
$$

with an uncertainty determined in the same way from the uncertainty in the area (provided by the least squares analysis). Here $E$ is a correction for pion decay, $J$ is the Jacobian of the transformation, and $T$ is an overall factor (which depends on the target thickness, the solid angle acceptance of the spectrometer, and the efficiency of the scattering monitor) determined such that the cross sections agree on the average with the predictions. The decay correction was

$$
E=\exp \left\{\Lambda m_{\pi} /(\tau p)\right\}
$$

in which $\Lambda$ is the mean path length between the target and the focal plane, $m_{\pi}$ and $\tau$ respectively are the rest mass and 1 ifetime of the charged pion, and $p$ is still the mean momentum of the pions scattered 

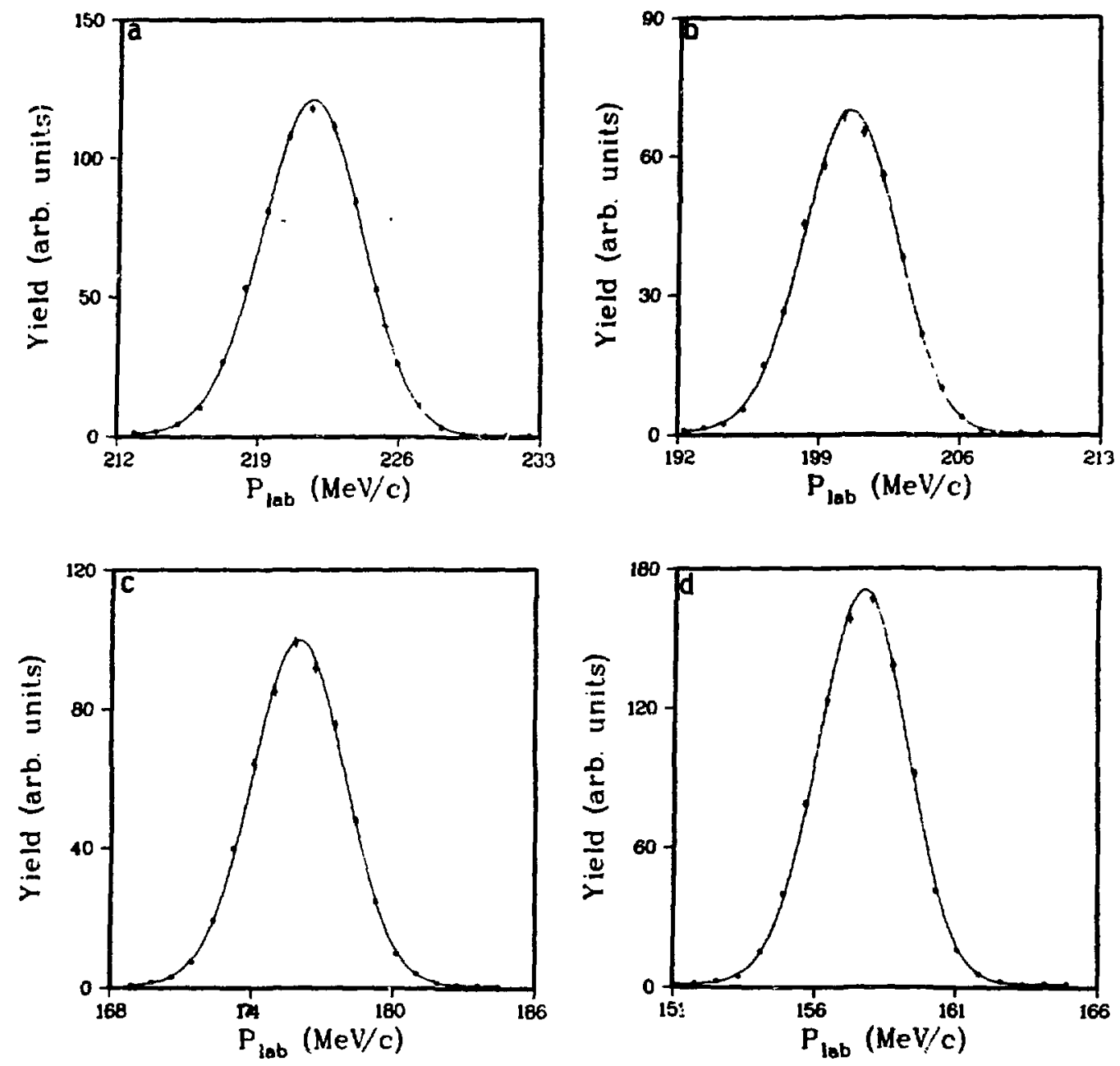

Fig. 12. Sample spectra for $\pi^{+} p+\pi^{+} p$. Spectra are for channels a) 1, b) 7, c) 10 and d) 17 . The curve was determined by the least squares fitting procedure. 
at the angle. The Jacobian was

$$
J=\left|\frac{d \cos \theta_{L A B}}{d \cos \theta}\right|=\left|p\left(T_{L A B} T-\gamma m_{\pi}^{2}\right) / m_{\pi}^{3}\right|
$$

where $T$ is the kinetic energy of the scattered pion and the subscript $L A B$ denotes in the laboratory frame. The overall factor $T$ was chosen to minimize the $x^{2}$,

$$
\left.x^{2}=\sum_{i}\left[y_{i}-f_{i}\right]^{2} /\left(\Delta y_{i}\right)^{2}+\left(\Delta f_{j}\right)^{2}\right\}
$$

where $y_{j}$ represents the measured cross section and $f_{j}$ is the value predicted by SCATPI using the beam momentum corrected for loss of energy entering the target, and the sum is over all angles. The beam momentum was the weighted average of the $\zeta_{3}$

$$
P_{\text {Beam }}=\left\{\sum_{i} \zeta_{3, j}\left(\Delta \zeta_{3}, j\right)^{-2}\right\} /\left\{\sum_{j}\left(\Delta \zeta_{3}, j\right)^{-2}\right\}
$$

where $\Lambda \zeta_{3}, j$ is the uncertainty in $\zeta_{j}, i$ provided by the least squares a $1-$ gorithm. The root mean square momentum variation $\Delta F$ was calculated in the same way from the $\zeta_{2, j}$ and $\Delta \zeta_{2, i}$.

The results of this analysis are presented in Table VII and the angular distribution is compared with the predicted cross section in Fig. 13. The figure reveals a $1.5 \%$ variation from forward to backward angles, which is not understood. Neither is an apparent systematic trend in the individual values of the beam momentum; however, the root mean square variation of the values is $0.13 \mathrm{MeV} / \mathrm{C}$, which may be a better estimate of the uncertainty in each measurement than the standard deviation provided by the fitting algoithm (which assumes that the form of the function $f\left(p_{i}\right)$ is correct). The estimates of the beam width are consistent within tiancertainties provided by the fitting algorithm. The results of the second angular survey, presented in Table VIII and Fig. 14, 
Table VII. Angular survey of $\pi^{+} p \rightarrow \pi^{+} p$ at $140 \mathrm{MeV}$. $P_{\text {beam }}$ and $\Delta P$ are the mean and root mean square variation of the incident momenta. $\theta_{l a b}$ is in degrees, $\delta \sigma / d \Omega$ is in $\mathrm{mb} / \mathrm{sr}$, and $\mathrm{P}_{\text {beam }}$ and $\Delta \mathrm{P}$ are in $\mathrm{MeV} / \mathrm{c}$.

\begin{tabular}{cccccc}
\hline${ }_{1 \mathrm{ab}}$ & $\cos \theta$ & $\frac{\mathrm{do}}{\mathrm{d} \Omega}$ & $P_{\text {beam }}$ & $\Delta P$ & $x^{2} / v$ \\
\hline 35 & 0.719 & $12.19 \pm 0.08$ & $242.22 \pm 0.02$ & $2.18 \pm 0.02$ & 4.36 \\
40 & 0.643 & $10.73 \pm 0.07$ & $242.30 \pm 0.02$ & $2.16 \pm 0.02$ & 3.37 \\
45 & 0.561 & $9.72 \pm 0.07$ & $242.29 \pm 0.02$ & $2.21 \pm 0.02$ & 2.28 \\
50 & 0.474 & $8.69 \pm 0.06$ & $242.19 \pm 0.02$ & $2.21 \pm 0.02$ & 2.60 \\
50 & 0.293 & $7.16 \pm 0.06$ & $242.15 \pm 0.03$ & $2.29 \pm 0.03$ & 1.11 \\
70 & 0.107 & $6.59 \pm 0.06$ & $242.08 \pm 0.03$ & $2.18 \pm 0.04$ & 2.50 \\
80 & -0.075 & $7.12 \pm 0.06$ & $242.01 \pm 0.03$ & $2.21 \pm 0.04$ & 2.79 \\
90 & -0.247 & $8.68 \pm 0.07$ & $242.18 \pm 0.03$ & $2.21 \pm 0.04$ & 1.89 \\
100 & -0.404 & $10.75 \pm 0.09$ & $241.95 \pm 0.03$ & $2.18 \pm 0.04$ & 1.17 \\
110 & -0.545 & $13.32 \pm 0.10$ & $241.99 \pm 0.03$ & $2.17 \pm 0.03$ & 2.61 \\
120 & -0.667 & $16.11 \pm 0.12$ & $241.91 \pm 0.03$ & $2.18 \pm 0.03$ & 1.19 \\
130 & -0.770 & $19.16 \pm 0.14$ & $242.03 \pm 0.02$ & $2.19 \pm 0.03$ & 2.57 \\
\hline \hline
\end{tabular}




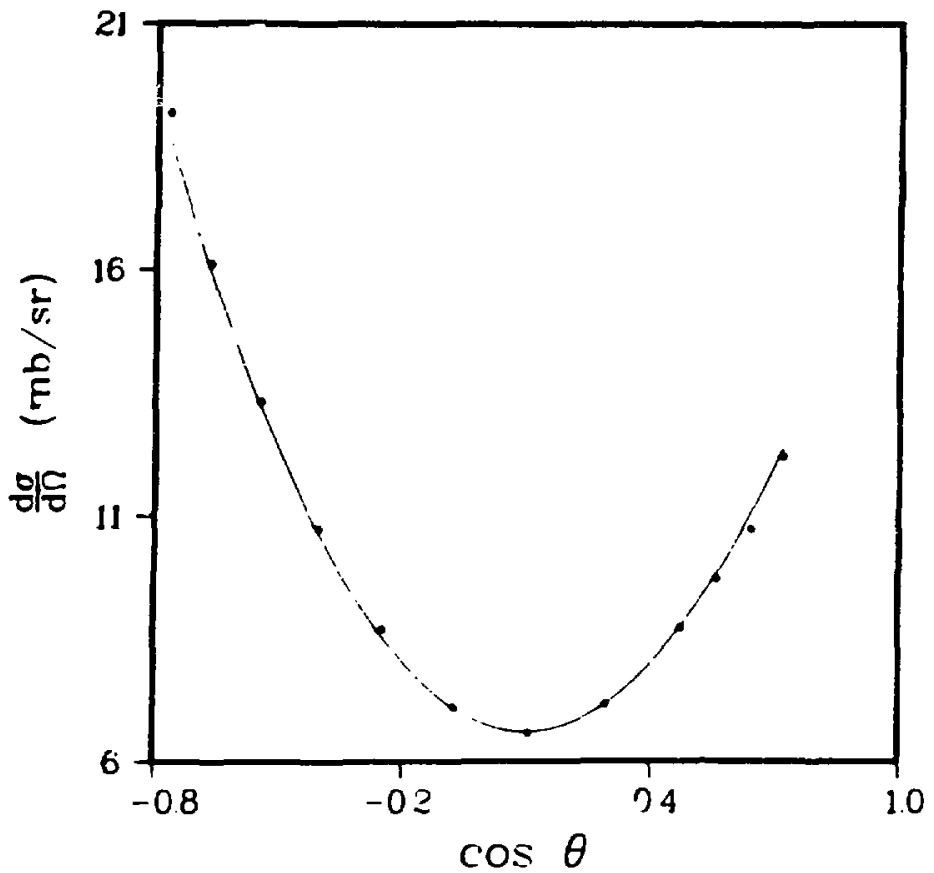

Fig. 13. Angular distribution of $\pi^{+} p \rightarrow \pi^{+} p$ at $140 \mathrm{MeV}$. The points have been normalized to agree as well as possible with the curve. The curve represents the interpolation of SCATPI. 
Table VIII. Repeat angular survey of $\pi^{+} p \rightarrow \pi^{+} p$ at $140 \mathrm{MeV}$. Entries are the same as in Table VII.

\begin{tabular}{rrrccc}
\hline$\theta_{L A B}$ & $\cos \theta$ & $\frac{d \sigma}{d \Omega}$ & $P_{\text {Beam }}$ & $\Delta P$ & $\chi^{2} / \nu$ \\
\hline 40 & 0.643 & $11.26 \pm 0.11$ & $242.76 \pm 0.03$ & $2.15 \pm 0.03$ & 4.04 \\
70 & 0.107 & $6.63 \pm 0.06$ & $242.59 \pm 0.04$ & $2.25 \pm 0.04$ & 1.61 \\
90 & -0.247 & $8.52 \pm 0.08$ & $242.79 \pm 0.04$ & $2.21 \pm 0.04$ & 1.06 \\
110 & -0.545 & $13.15 \pm 0.12$ & $242.45 \pm 0.04$ & $2.15 \pm 0.04$ & 5.09 \\
130 & -0.770 & $18.78 \pm 0.17$ & $242.24 \pm 0.03$ & $2.13 \pm 0.04$ & 2.96 \\
\hline
\end{tabular}

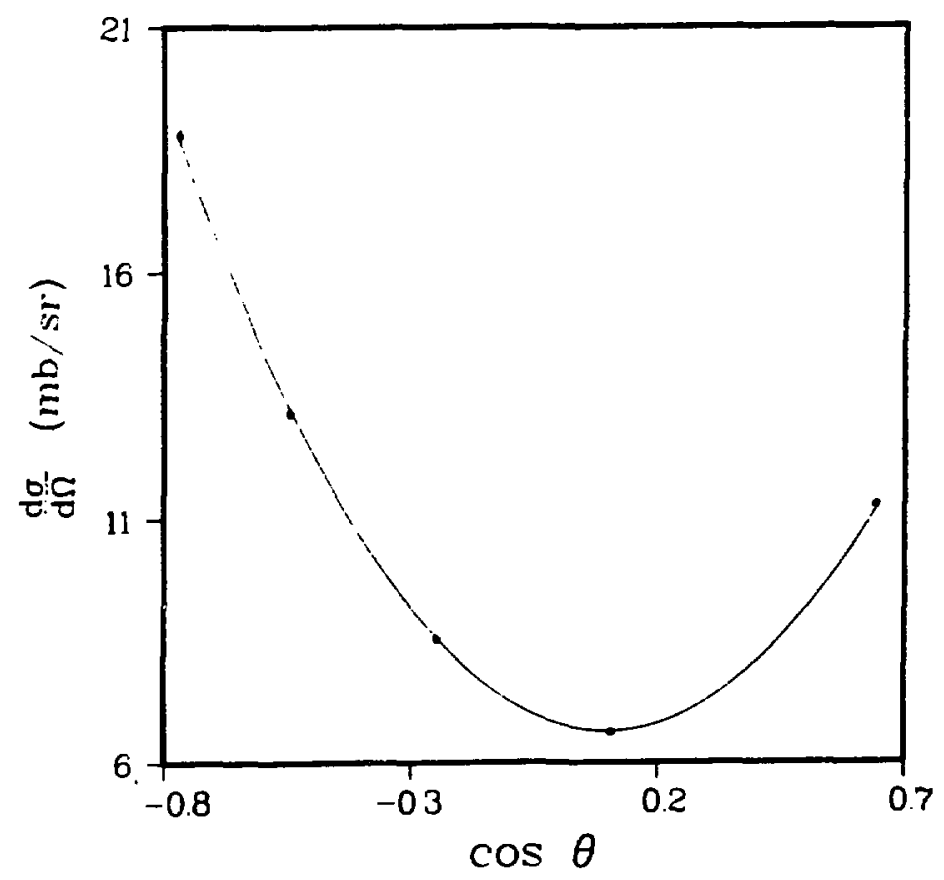

Fig. 14. Repeat of angular distribution of $\pi^{+} p \rightarrow \pi^{+} p$ at $140 \mathrm{MeV}$. The points have been normalized to agree as well as possible with the curve. The curve represents the interpolation of SCATPI. 
show neither of the trends evident in the first study. Only five angles were included the second time; however, it is noteworthy that the measurements reproduce the predicted angular distribution. The target flask had been repositioned during a two week interruption, but it is not clear that this is the only or the complete explanation. The root mean square variation in the estimates of the beam momentum is 0.23 $\mathrm{MeV} / \mathrm{c}$, again hinting that the statistical uncertainty is too small. The beam momentum was determined to be $242.11 \mathrm{MeV} / \mathrm{c}$ and $242.57 \mathrm{MeV} / \mathrm{c}$ for the two studies, suggesting that it is reproducible to about $0.2 \%$.

\section{$\pi^{-p}$ Elastic Scattering}

Elastic scattering of the $\pi^{-}$meson from protons was measured for each $\pi^{-}$beam employed in the $\pi^{+}$production study. These data were analyzed very much the same as the $\pi^{+} p$ data in the preceding section. The effective target thickness, the spectrometer solid angle acceptance to lowest order and the efficiency of the scattering monitor were thus determined together, avoiding the chore of evaluating each separately. This analysis also provided the momentum distribution for each of the incident $\pi^{-}$beams, which are used in the analysis of the reaction measurements.

Measurements for $\pi^{-} p$ elastic scattering were made beginning as far forward as permitted by the upper momentum limit of the spectrometer and proceding to $130^{\circ}$ in $10^{\circ}$ increments in the laboratory. These data were analyzed in the same manner as described in the previous section except that, in the function fitted to the spectra, the error function term was excluded. Due to the $\Delta p / p$ of $4 \%$ for the $\pi^{-}$beams, the background jevels above and below the elastic peak were inadequately defined to include the 
error function term while allowing the peak to be skewed. Also, as described in Chapter IV, the cross section predictions used to determina I for the two highest energy beams were not provided by SCATPI, b.It rather by a graphical method.

Some example spectra are presented for each energy in Fig. 15 through 20. The results of the analyses of the spectra are displayed in Table IX and Fig. 21 for $229 \mathrm{MeV}$, Table X and Fig. 22 for $254 \mathrm{MeV}$, Table XI and Fig. 23 for $279 \mathrm{MeV}$, Table XII and Fig. 24 for $292 \mathrm{MeV}$, Tables XIII and XIV for $330 \mathrm{MeV}$ and Tables XV and XVI for $356 \mathrm{MeV}$. Table XVII is a distillation of these results, presenting the values of $T, P_{\text {Beam }}, \Delta P$ and their root mean square variations for each beam. The variations in $T$ for the lowest four energies are consistent with the 1 to $1 \frac{1}{2} \%$ accuracy of the cross section measurements. For the two higher energies the agreement is lesser, presumably reflecting the consistency of the graphical interpolation of the cross sections. The variations in $\mathrm{P}_{\text {Beam }}$, the beam momentum, are about $0.07 \%$, more than twice the statistically expected $0.03 \%$. This may indicate the level of adequacy of the form fitted to the spectra, the computation of $\zeta_{3}$ from the mean momentum of the scattered $\pi$ mesons, or other factors. The values of $\Delta P$ are consistent to within their statistical uncertainties. The values of the beam momentum and root mean square variation corrected for loss of energy in the target are listed in Table XVIII. Along with $\mathrm{T}$ from Table XVII, these were used in analyzing the reaction data. 

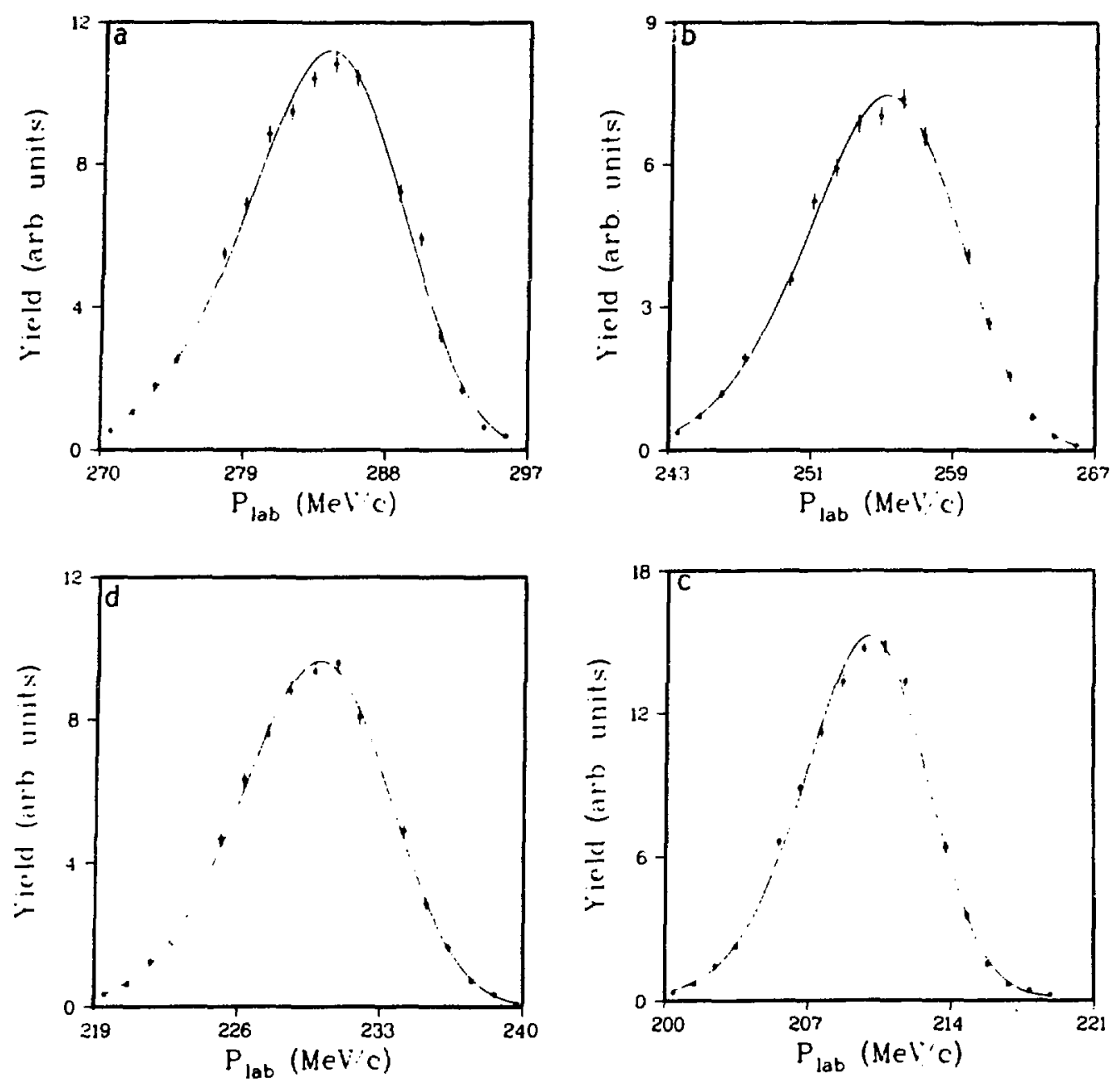

Fig. 15. Sample spectra for $\pi^{-p} \rightarrow-\pi-p$ at $229 \mathrm{MeV}$. Spectra are for the Taboratory angles a) $60^{\circ}$, b) $80^{\circ}$, c) $100^{\circ}$ and d) $120^{\circ}$. The curve was determined by. the least squares fitting procedure. 

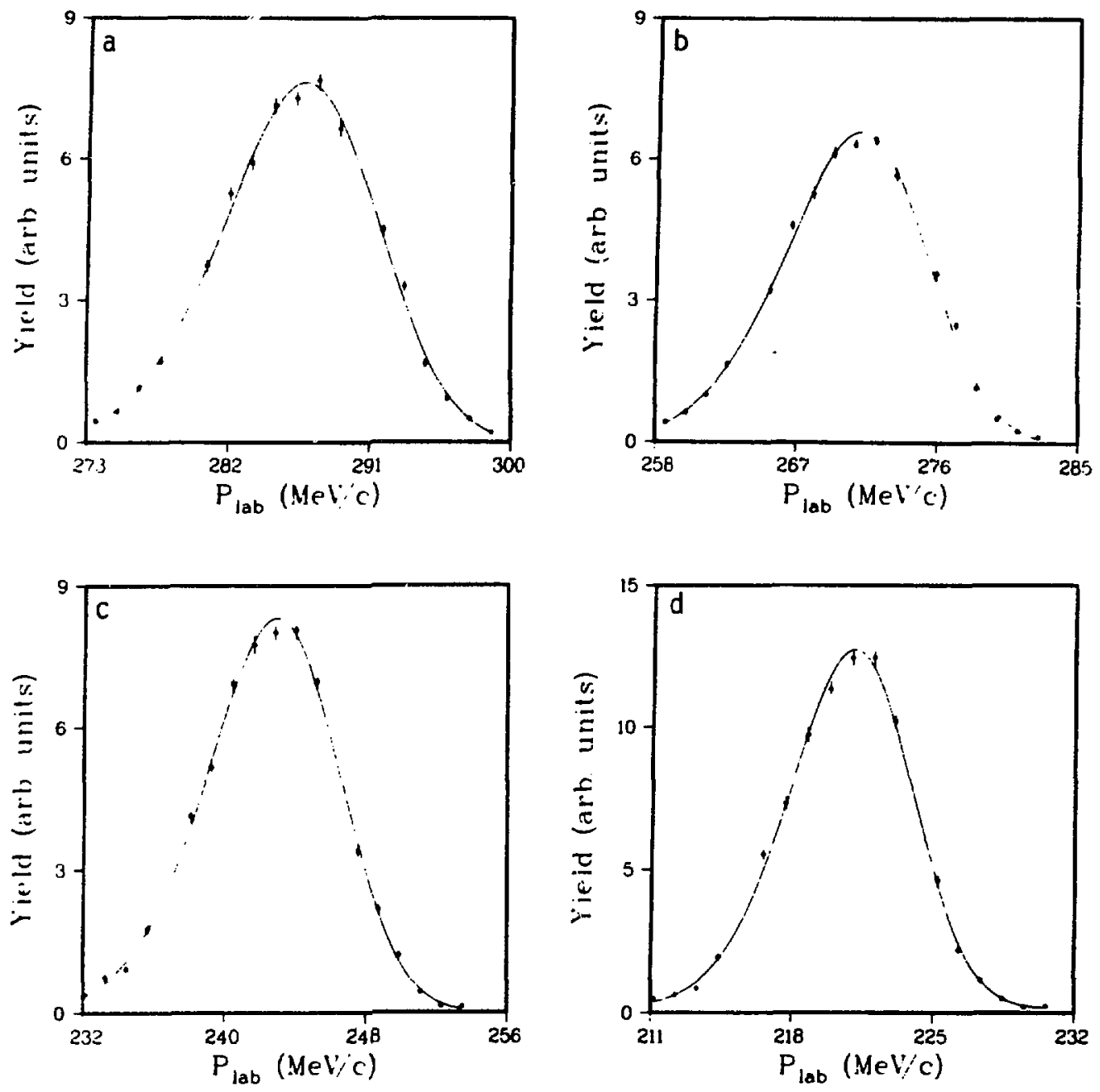

Fig. 16. Sample spectra for $\pi^{-p \rightarrow \pi^{-p}}$ at $254 \mathrm{MeV}$. Spectra are for the laboratory angles a) $70^{\circ}$, b) $80^{\circ}$, c) $100^{\circ}$ and d) $120^{\circ}$. The curve was determined by the least squares fitting procedure. 


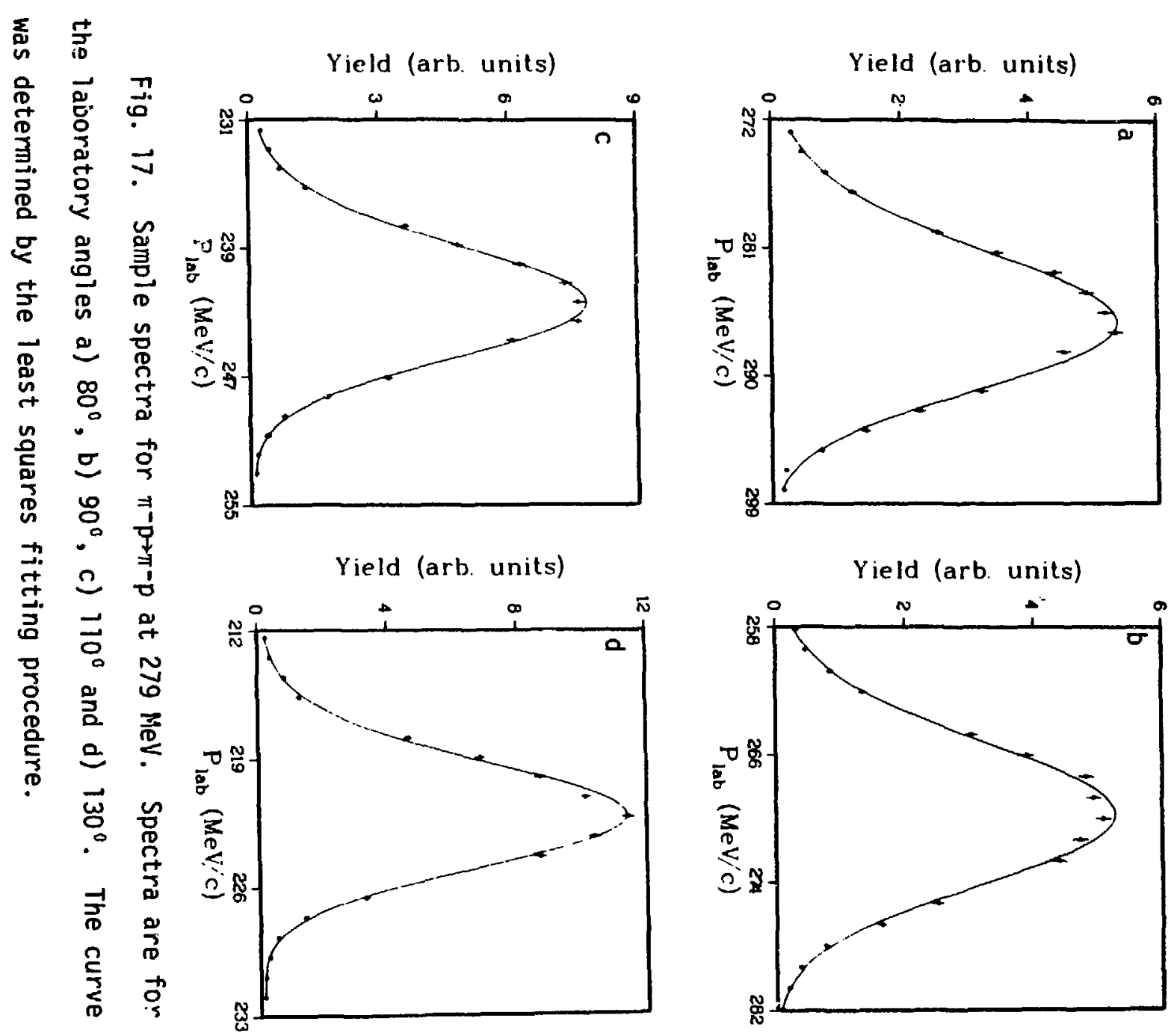




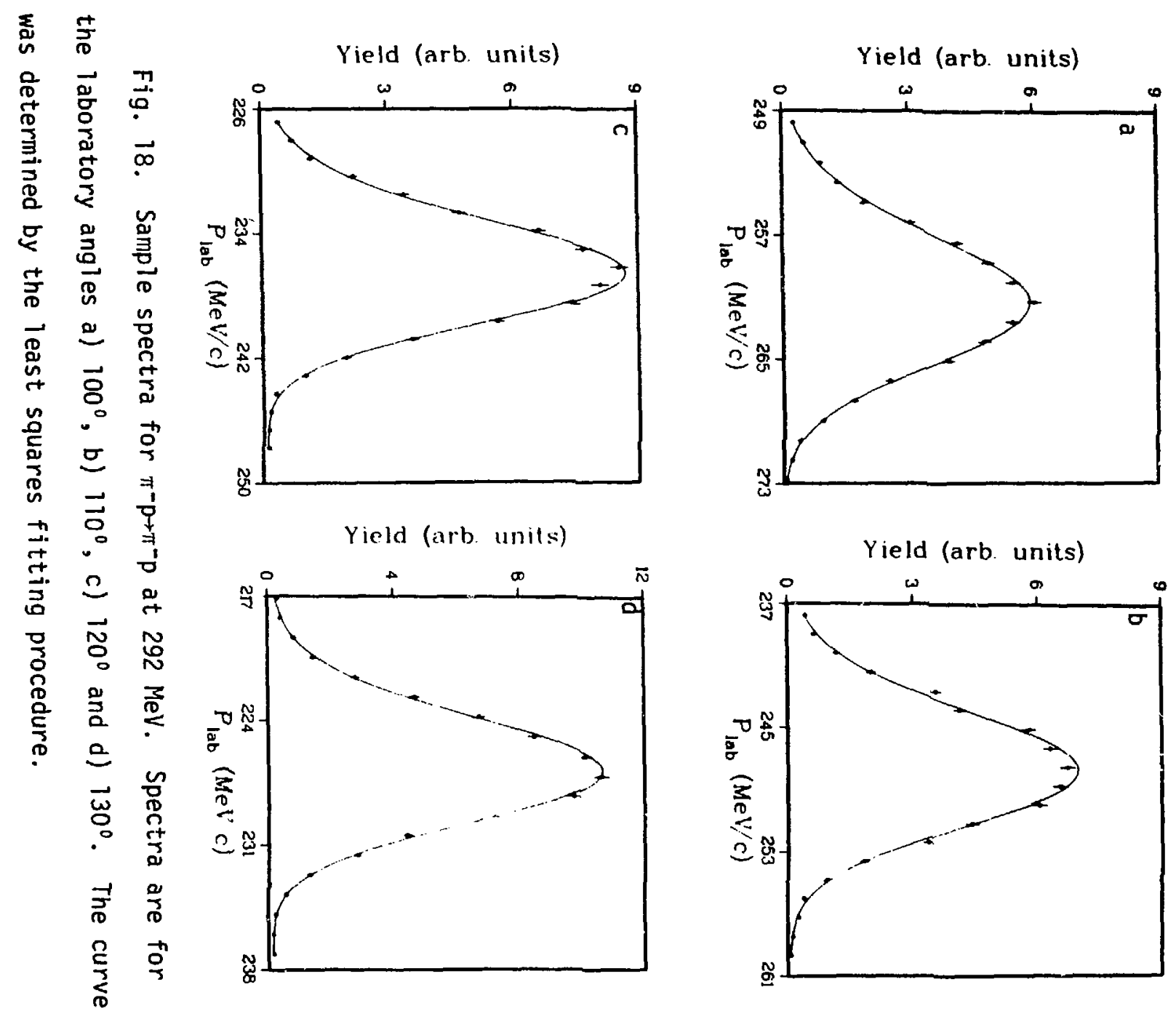



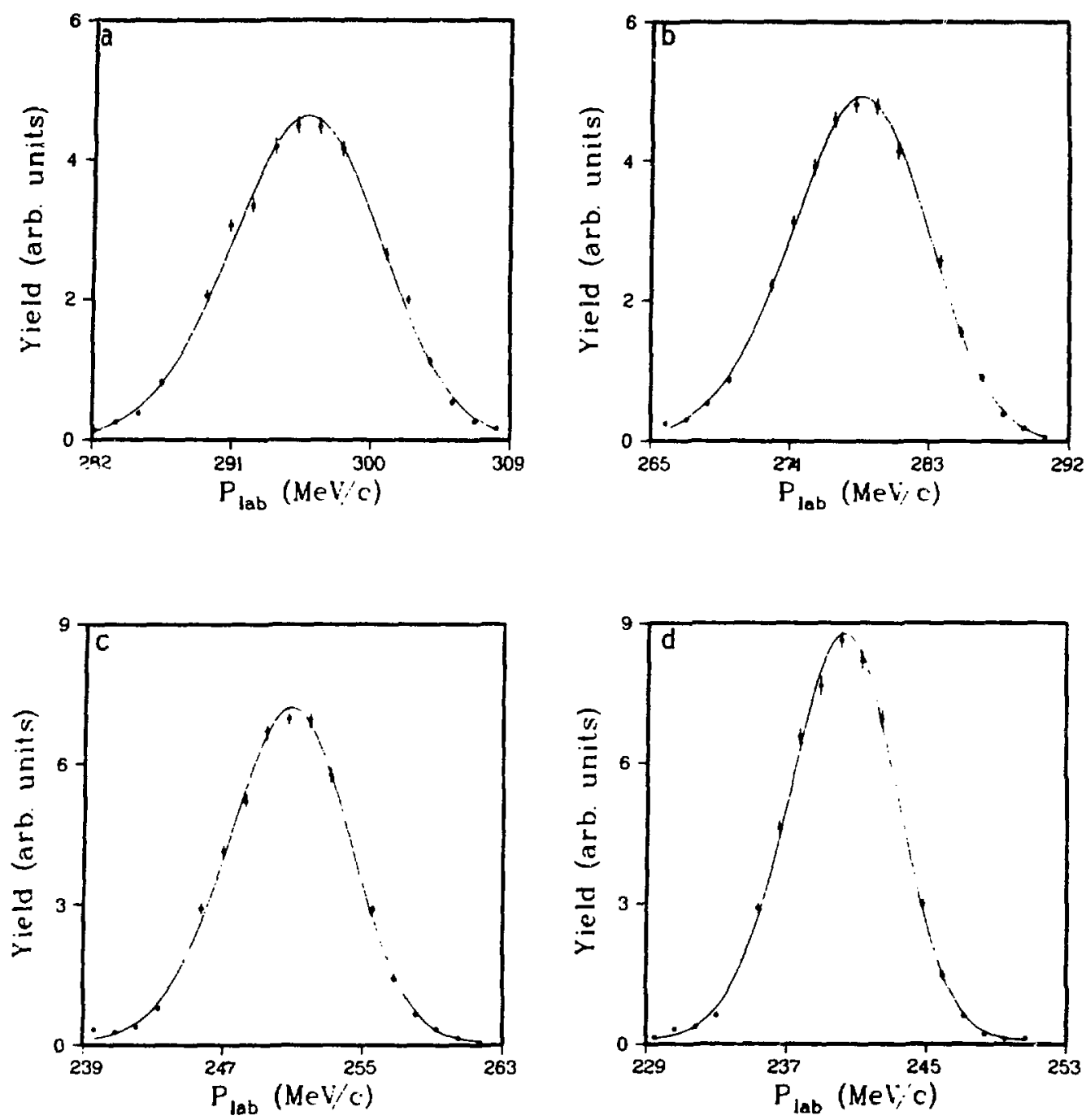

Fig. 19. Sample spectra for $\pi^{-p \rightarrow \pi-p}$ at $330 \mathrm{MeV}$. Spectra are for the laboratory angles a) $90^{\circ}$, b) $100^{\circ}$, c) $120^{\circ}$ and d) $130^{\circ}$. The curve was determined by the least squares fitting procedure. 

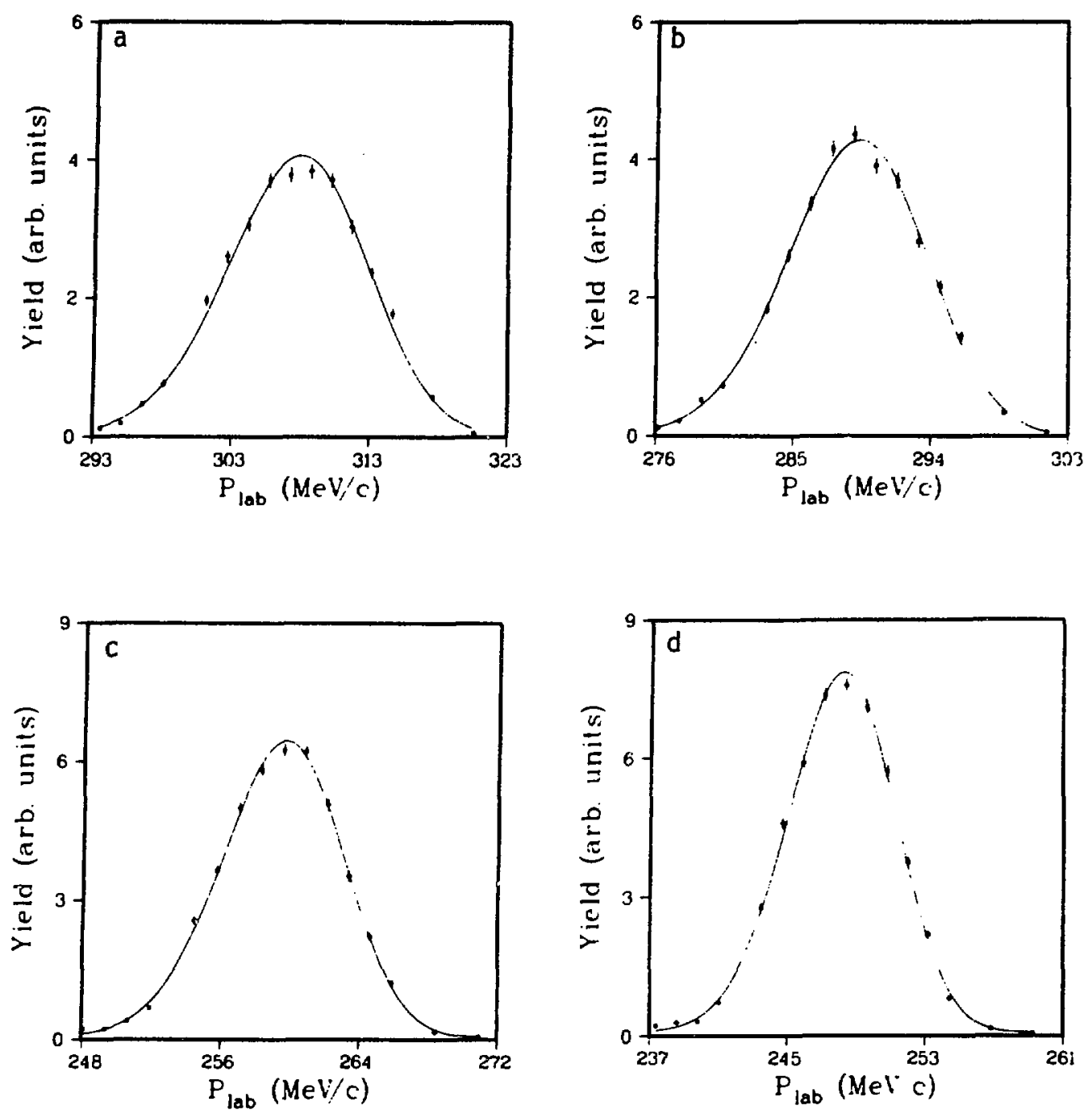

Fig. 20. Sample spectra for $\pi-p \rightarrow \pi-p$ at $356 \mathrm{MeV}$. Spectra are for the laboratory angles a) $90^{\circ}$, b) $100^{\circ}$, c) $120^{\circ}$ and d) $130^{\circ}$. The curve was determined by the least squares fitting procedure. 
Table IX. Results from $\pi^{-} p \rightarrow \pi^{-} p$ at $229 \mathrm{MeV}$. Entries are the same as in Table VII.

\begin{tabular}{rcccccc}
\hline${ }_{\text {LAB }}$ & $\cos \theta$ & $\frac{d \sigma}{d \Omega}$ & $P_{\text {Beam }}$ & $\Delta P$ & $\chi^{2} / \nu$ \\
\hline 60 & 0.241 & $0.878 \pm 0.020$ & $341.20 \pm 0.09$ & $5.98 \pm 0.17$ & 2.75 \\
70 & 0.052 & $0.696 \pm 0.014$ & $340.81 \pm 0.10$ & $5.96 \pm 0.15$ & 1.82 \\
80 & -0.129 & $0.641 \pm 0.013$ & $340.62 \pm 0.10$ & $6.00 \pm 0.16$ & 0.92 \\
90 & -0.298 & $0.716 \pm 0.014$ & $341.18 \pm 0.10$ & $5.91 \pm 0.16$ & 0.92 \\
100 & -0.450 & $0.894 \pm 0.014$ & $340.94 \pm 0.10$ & $6.05 \pm 0.13$ & 0.80 \\
110 & -0.583 & $1.129 \pm 0.017$ & $340.99 \pm 0.09$ & $6.10 \pm 0.12$ & 1.55 \\
120 & -0.697 & $1.412 \pm 0.019$ & $341.18 \pm 0.09$ & $5.87 \pm 0.10$ & 2.17 \\
130 & -0.791 & $1.666 \pm 0.020$ & $340.96 \pm 0.08$ & $5.49 \pm 0.09$ & 2.95
\end{tabular}

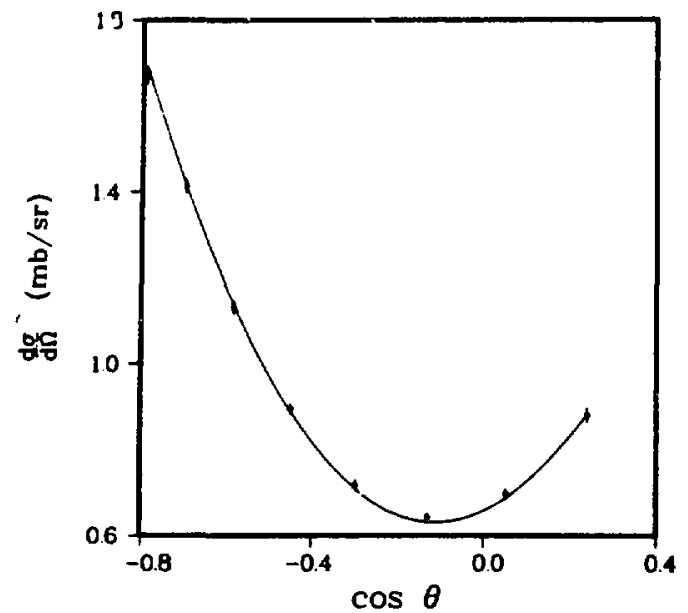

Fig. 21. Angular distribution of $\pi^{-} p \rightarrow \pi^{-} p$ at $229 \mathrm{MeV}$. The points have been normalized to agree as well as possible with the curve. The curve represents the interpolation of SCATPI. 
Table $X$. Results from $\pi^{-} p \rightarrow \pi^{-} p$ at $254 \mathrm{MeV}$. Entries are the same as in Table VII.

\begin{tabular}{cccccc}
\hline$\theta_{L A B}$ & $\cos \theta$ & $\frac{d \sigma}{d \Omega}$ & $P_{\text {Beam }}$ & $\Delta P$ & $\chi^{2} / \nu$ \\
\hline 70 & 0.037 & $0.605 \pm 0.012$ & $368.14 \pm 0.09$ & $6.35 \pm 0.16$ & 1.75 \\
80 & -0.144 & $0.543 \pm 0.009$ & $367.68 \pm 0.10$ & $6.40 \pm 0.15$ & 2.15 \\
90 & -0.311 & $0.592 \pm 0.010$ & $367.91 \pm 0.09$ & $6.40 \pm 0.15$ & 1.87 \\
100 & -0.461 & $0.720 \pm 0.010$ & $367.72 \pm 0.04$ & $6.27 \pm 0.13$ & 1.93 \\
110 & -0.592 & $0.922 \pm 0.12$ & $367.88 \pm 0.09$ & $6.26 \pm 0.12$ & 2.29 \\
120 & -0.704 & $1.104 \pm 0.014$ & $368.14 \pm 0.09$ & $6.07 \pm 0.11$ & 2.11 \\
130 & -0.797 & $1.379 \pm 0.016$ & $367.92 \pm 0.09$ & $6.00 \pm 0.09$ & 2.48 \\
\hline \hline
\end{tabular}

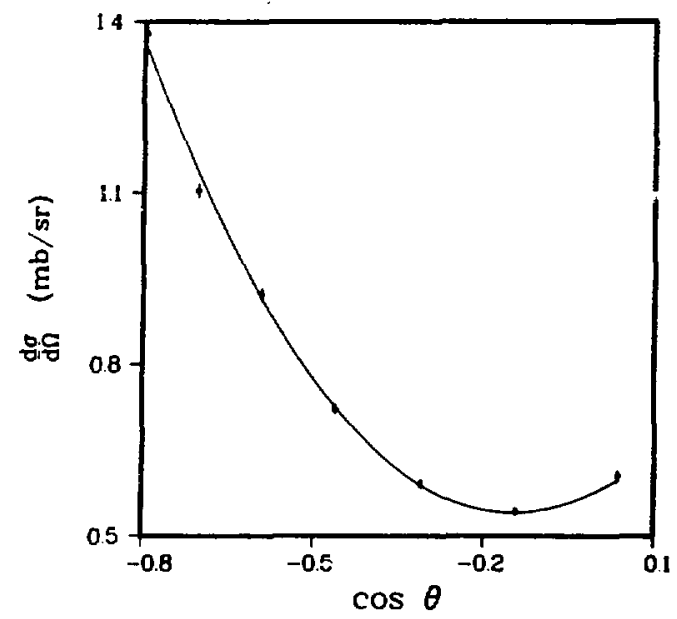

Fig. 22. Angular distribution of $\pi^{-} p \rightarrow \pi^{-} p$ at $254 \mathrm{MeV}$. The points have been normalized to agree as well as possible with the curve. The curve represents the interpolation of SCATPI. 
Table XI. Results from $\pi^{-} p \rightarrow \pi^{-} p$ at $279 \mathrm{MeV}$. Entries are the same as in Table VII.

\begin{tabular}{rccccc}
\hline \hline$\theta_{L A B}$ & $\cos \theta$ & $\frac{d \sigma}{d \Omega}$ & $P_{\text {Beam }}$ & $\Delta P$ & $\chi^{2} / v$ \\
\hline 80 & -0.158 & $0.501 \pm 0.010$ & $394.75 \pm 0.10$ & $7.13 \pm 0.19$ & 2.82 \\
90 & -0.324 & $0.509 \pm 0.008$ & $394.81 \pm 0.10$ & $7.05 \pm 0.15$ & 2.10 \\
100 & -0.472 & $0.608 \pm 0.008$ & $394.74 \pm 0.10$ & $6.76 \pm 0.14$ & 2.24 \\
110 & -0.601 & $0.747 \pm 0.009$ & $394.76 \pm 0.09$ & $6.62 \pm 0.12$ & 1.85 \\
120 & -0.711 & $0.914 \pm 0.010$ & $394.86 \pm 0.09$ & $6.68 \pm 0.10$ & 1.10 \\
130 & -0.802 & $1.084 \pm 0.011$ & $394.87 \pm 0.08$ & $6.56 \pm 0.09$ & 3.14 \\
90 & -0.324 & $0.513 \pm 0.012$ & $394.75 \pm 0.15$ & $7.18 \pm 0.24$ & 0.82 \\
110 & -0.601 & $0.769 \pm 0.013$ & $394.77 \pm 0.13$ & $6.88 \pm 0.17$ & 2.13 \\
130 & -0.802 & $1.059 \pm 0.015$ & $394.82 \pm 0.11$ & $6.47 \pm 0.12$ & 3.11 \\
\hline \hline
\end{tabular}

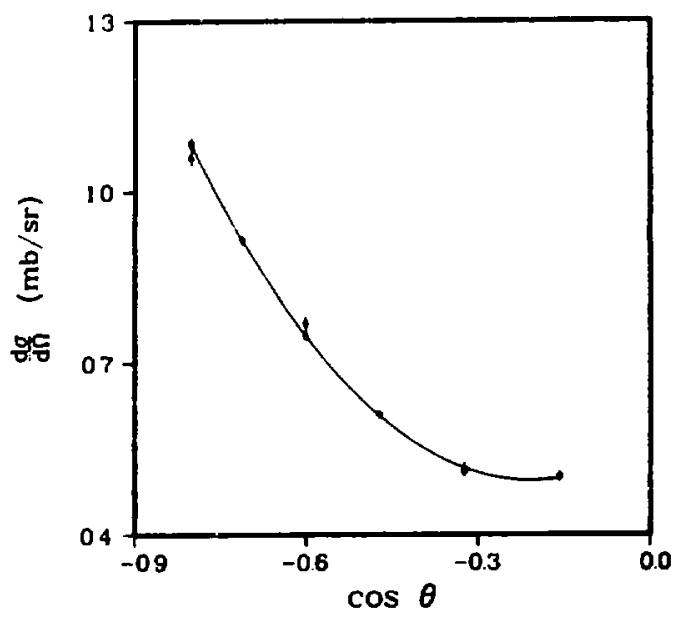

Fig. 23. Angular distribution of $\pi^{-} p \rightarrow \pi^{-} p$ at $279 \mathrm{MeV}$. The points have been normalized to agree as well as possible with the curve. The curve represents the interpolation of SCATPI. 
Table XII. Results from $\pi^{-} p \rightarrow \pi^{-} p$ at $292 \mathrm{MeV}$. Entries are the same as in Table VII.

\begin{tabular}{llllll}
\hline$\theta_{L A B}$ & $\cos \theta$ & $\frac{d \sigma}{d \Omega}$ & $P_{\text {Beam }}$ & $\Delta P$ & $x^{2} / \nu$ \\
\hline
\end{tabular}

(a) First beam

$\begin{array}{llllll}100 & -0.478 & 0.572 \pm 0.009 & 408.17 \pm 0.13 & 7.52 \pm 0.18 & 0.68 \\ 110 & -0.606 & 0.672 \pm 0.009 & 407.83 \pm 0.12 & 7.89 \pm 0.15 & 2.11 \\ 120 & -0.714 & 0.825 \pm 0.010 & 408.23 \pm 0.11 & 7.19 \pm 0.12 & 1.14 \\ 130 & -0.804 & 0.985 \pm 0.011 & 408.35 \pm 0.10 & 6.90 \pm 0.11 & 1.03\end{array}$

(b) Second beam

\begin{tabular}{llllll}
100 & -0.478 & $0.568 \pm 0.010$ & $408.43 \pm 0.13$ & $7.05 \pm 0.20$ & 1.84 \\
110 & -0.606 & $0.679 \pm 0.010$ & $407.80 \pm 0.13$ & $6.80 \pm 0.15$ & 1.77 \\
120 & -0.714 & $0.813 \pm 0.011$ & $408.30 \pm 0.12$ & $6.76 \pm 0.19$ & 2.44 \\
130 & -0.804 & $0.998 \pm 0.013$ & $407.90 \pm 0.11$ & $6.81 \pm 0.12$ & 1.57 \\
\hline
\end{tabular}
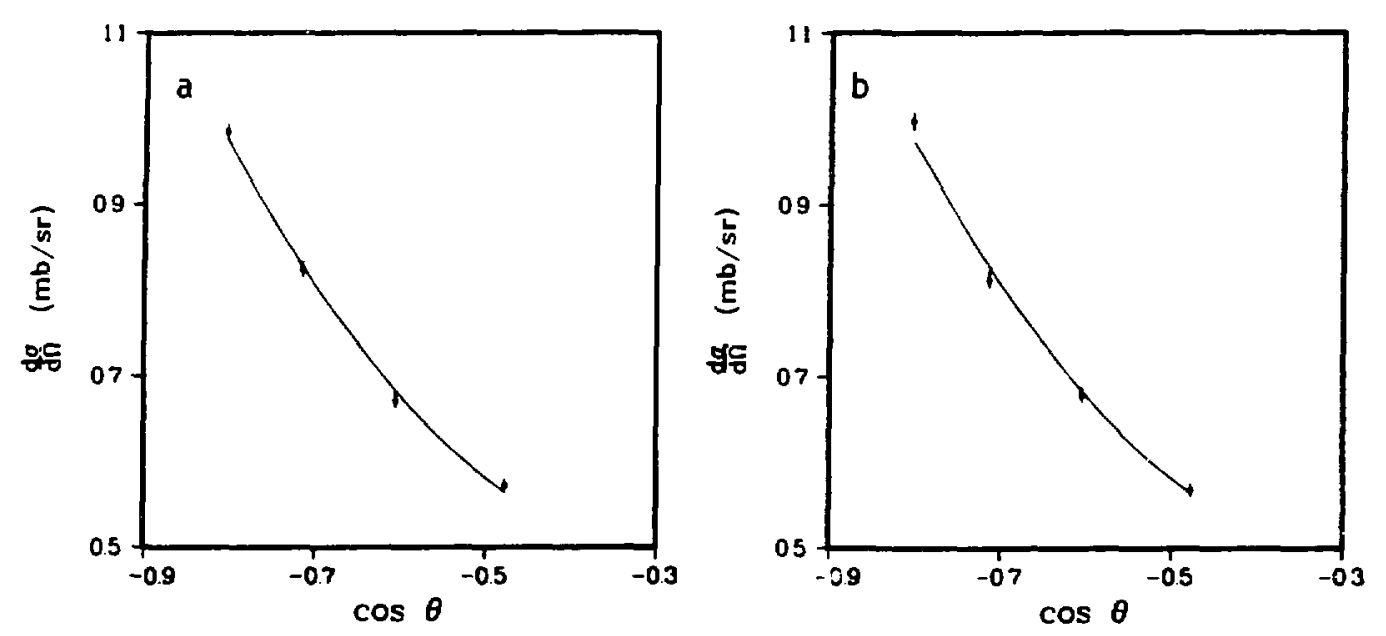

Fig. 24. Angular distribution of $\pi^{-} p \rightarrow \pi^{-} p$ at $292 \mathrm{MeV}$. a) first beam, b) second beam. The points have been normalized to agree as well as possible with the curve. The curve represents the interpolation of SCATPI. 
Table XIII. Results from $\pi^{-} p \rightarrow \pi^{-} p$ spectra at $330 \mathrm{MeV}$. Entries are the same as in Table VII.

\begin{tabular}{rccccc}
\hline$\theta_{\text {LAB }}$ & $\cos \theta$ & $\frac{\mathrm{d} \sigma}{\mathrm{d} \Omega}$ & $P_{\text {Beam }}$ & $\Delta P$ & $\chi^{2} / \nu$ \\
\hline 90 & -0.349 & $0.423 \pm 0.008$ & $449.72 \pm 0.13$ & $7.77 \pm 0.25$ & 2.18 \\
100 & -0.494 & $0.467 \pm 0.007$ & $448.98 \pm 0.13$ & $8.03 \pm 0.20$ & 1.04 \\
110 & -0.619 & $0.564 \pm 0.008$ & $448.33 \pm 0.13$ & $7.93 \pm 0.18$ & 0.99 \\
120 & -0.725 & $0.671 \pm 0.009$ & $449.18 \pm 0.13$ & $7.66 \pm 0.16$ & 2.66 \\
130 & -0.811 & $0.803 \pm 0.010$ & $449.38 \pm 0.12$ & $7.54 \pm 0.14$ & 2.06 \\
90 & -0.349 & $0.422 \pm 0.011$ & $449.44 \pm 0.18$ & $8.16 \pm 0.34$ & 2.60 \\
110 & -0.619 & $0.553 \pm 0.008$ & $448.54 \pm 0.14$ & $7.71 \pm 0.20$ & 1.70 \\
130 & -0.811 & $0.802 \pm 0.010$ & $448.73 \pm 0.12$ & $7.64 \pm 0.14$ & 2.78 \\
\hline \hline
\end{tabular}

Table XIV. Interpolated cross sections for $\pi^{-} p \rightarrow \pi^{-} p$ at $330 \mathrm{MeV}$. These cross sectins were used to determine the normalization of the cross sections reported in Table XIII. Units are mb/sr.

\begin{tabular}{cc}
\hline $\cos \theta$ & $\frac{\mathrm{d} \sigma}{\mathrm{d} \Omega}$ \\
\hline-0.348 & $0.436 \pm 0.020$ \\
-0.494 & $0.471 \pm 0.019$ \\
-0.619 & $0.557 \pm 0.022$ \\
-0.725 & $0.679 \pm 0.030$ \\
-0.812 & $0.775 \pm 0.035$ \\
\hline
\end{tabular}


Table XV. Results from $\pi^{-} p \rightarrow \pi^{-} p$ spectra at $356 \mathrm{MeV}$. Entries are the same as in Table VII.

\begin{tabular}{rccccc}
\hline \hline$\theta_{L A B}$ & $\cos \theta$ & $\frac{d \sigma}{d \Omega}$ & $P_{\text {Beam }}$ & $\Delta P$ & $x^{2} / \nu$ \\
\hline 90 & -0.360 & $0.405 \pm 0.008$ & $476.11 \pm 0.16$ & $8.38 \pm 0.32$ & 1.19 \\
100 & -0.503 & $0.435 \pm 0.008$ & $475.61 \pm 0.17$ & $8.38 \pm 0.28$ & 1.26 \\
110 & -0.627 & $0.522 \pm 0.007$ & $475.25 \pm 0.14$ & $8.35 \pm 0.20$ & 2.49 \\
120 & -0.731 & $0.637 \pm 0.008$ & $474.94 \pm 0.11$ & $7.85 \pm 0.13$ & 3.41 \\
130 & -0.816 & $0.763 \pm 0.008$ & $475.25 \pm 0.12$ & $7.92 \pm 0.16$ & 2.31 \\
\hline
\end{tabular}

Table XVI. Interpolated cross section for $\pi^{-} p \rightarrow \pi^{-} p$ at $356 \mathrm{MeV}$. These cross sections were used to determine the normalization of the cross sections reported in Table $\mathrm{XV}$. Units are mb/sr.

\begin{tabular}{ll}
\hline $\cos \theta$ & $\frac{\mathrm{d} \sigma}{\mathrm{d} \Omega}$ \\
-0.360 & $0.400 \pm 0.016$ \\
-0.503 & $0.443 \pm 0.018$ \\
-0.627 & $0.527 \pm 0.021$ \\
-0.731 & $0.642 \pm 0.026$ \\
-0.816 & $0.740 \pm 0.035$ \\
\hline
\end{tabular}


Table XVII. Results from the study of $\pi^{-} 0 \rightarrow \pi^{-} p$. T $T_{\text {Beam }}$ is the kinetic energy of the incident beam in MeV, $T$ is the normalization factor in (mb/sr) (monitor counts/ $\pi$ ), $P_{\text {Beam }}$ is the mean mumentum of the incident beam in $M e V / c$, and $\Delta P$ is the root mean square variation in the incident momentum in $\mathrm{HeV} / \mathrm{c}$. The quoted uncertainties in $P_{\text {Beam }}$ and $\triangle P$ are the root mean square deviations of the individual values at each energy.

\begin{tabular}{|c|c|c|c|c|}
\hline$T_{\text {Beam }}$ & & $\tau$ & $P_{\text {Beam }}$ & $\Delta P$ \\
\hline 229 & & $25.59 \pm 0.29$ & $341.00 \pm 0.20$ & $5.85 \pm 0.19$ \\
\hline 254 & & $23.54 \pm 0.38$ & $367.92 \pm 0.18$ & $6.20 \pm 0.16$ \\
\hline 279 & & $24.68 \pm 0.33$ & $394.80 \pm 0.05$ & $6.70 \pm 0.26$ \\
\hline 292 & $a$ & $23.10 \pm 0.31$ & $403.18 \pm 0.23$ & $7.16 \pm 0.43$ \\
\hline 292 & $b$ & $23.82 \pm 0.42$ & $408.09 \pm 0.30$ & $6.82 \pm 0.13$ \\
\hline 330 & & $21.68 \pm 1.07$ & $449.02 \pm 0.48$ & $7.73 \pm 0.21$ \\
\hline 356 & & $22.45 \pm 0.51$ & $475.33 \pm 0.45$ & $8.04 \pm 0.27$ \\
\hline
\end{tabular}


Table XVIII. Incident momentum distributions corrected for loss of energy in the target. Units are MeV/c.

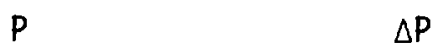

$\begin{array}{ll}340.64 & 5.85 \\ 367.57 & 6.20 \\ 394.46 & 6.70 \\ 407.83 & 7.17 \\ 407.75 & 6.83 \\ 448.69 & 7.74 \\ 474.99 & 8.04\end{array}$


CHAPTER IV

INTERPOLATON OF THE $\pi^{ \pm}$p ELASTIC DIFFERENTIAL CROSS SECTIONS

This chapter describes the interpolation of the differential cross section for $\pi p$ elastic scattering. Below $300 \mathrm{MeV}$, energy independent phase shifts were fitted to plausible functions of momentum, and the interpolated phase shifts were used to calculate the differential cross section. A less elegant method of interpolation was necessitated at $330 \mathrm{MeV}$ and $356 \mathrm{MeV}$ by the limited accuracy and sparsity of the data above $300 \mathrm{MeV}$. A graphical method provided the cross sections at these two energies.

\section{Interpolation Beiow $300 \mathrm{MeV}$}

Below $300 \mathrm{MeV}$, the elastic differential cross section was interpolated in SCATPI. 10 The subroutine was derived from the phase shift analysis of Carter, Bugg and Carter ${ }^{11}$ which in turn had been based primarily on the differential cross sections for $\pi^{ \pm} p$ elastic scattering measured by Bussey, Carter, Dance, Bugg, Carter and Smith, ${ }^{12}$ but also on total cross sections, integrated charge exchange cross sections and polarization measurements. ${ }^{13-16}$ Their anaiysis provided eleven sets of phase shifts for the $\pi^{+} p$ system between $95 \mathrm{MeV}$ and $310 \mathrm{MeV}$, and nine for the $\pi^{-p}$ system between $89 \mathrm{MeV}$ and $292 \mathrm{MeV}$. These were fitted by least squares to plausible functions of momentum which had as few free parameters as would permit a reasonable $\chi^{2} / v$. Thus the phase shifts interpolated with these functions should be physically reasonable.

As a minimum test of its accuracy, the predictions of SCATPI have 
been compared to the measurements from which it was derived and have typically differed by the following amounts:

$\begin{array}{llll} & \pi^{+} p & \pi^{-} p \\ \text { differential elastic cross section } & 2 \% & & 2 \frac{1}{2} \% \text { below } 260 \mathrm{MeV} \\ \text { total cross section } & 2 \% & 4 \% \text { above } 260 \mathrm{MeV} \\ \begin{array}{l}\text { integrated charge exchange } \\ \quad \text { cross sections }\end{array} & \frac{12 \%}{2} \% & 1 \% & \\ & -- & 1 \%\end{array}$

These differences are generally reasonable considering the uncertainties in the measurements, except for the $\pi^{-p}$ elastic differential cross section at higher energies where the phase shift analysis seems in some difficulty. Predictions for the polarization parameter also agree reasonably with the measurements. Predictions of SCATPI have also been compared to measurements that have become available since the subroutine was completed, and have agreed with similar precision.

SCATPI is based upon a set of phase shifts that are interpolated from the analysis of Carter, Bugg and Carter, and necessarily follows the same scheme employed by those authors in relating the phase shifts to the differential cross section. The scattering amplitude in the center of momentum system is

$$
F=f(\theta, k)+g(\theta, k) \hat{n} \cdot \vec{\sigma} .
$$

Here $\vec{\sigma}$ are the usual Pauli matrices, $\theta$ is the angle between the initial and final wave vectors, $\vec{k}_{\mathbf{j}}$ and $\vec{k}_{f}$, respectively, $k=\left|\vec{k}_{j}\right|$ is the initial wave number, and $\vec{r}_{i}$ is a unit vector orthogonal to both $\vec{k}_{j}$ and $\vec{k}_{f}$, $\hat{n}=\vec{k}_{f} \times \vec{k}_{j} /\left|\vec{k}_{f} \times \vec{k}_{j}\right|$. The functions $f(\theta, k)$ and $g(\theta, k)$ are commonly called the spin-nonflip and spin-flip scattering amplitudes, respectively. If the spin of the final state is not observed and the initial 
state is unpolarized, the differential cross section is

$$
\frac{d \sigma}{d \Omega}=|f(\theta, k)|^{2}+|g(\theta, k)|^{2} \text {. }
$$

Since the Coulomb interaction with its long range cannot be ignored when both the pion and nucleon are charged, many terms must be kept in a partial wave expansion to adequately represent the resulting scattering amplitude; however, only a few contain significant contribution from the strong interaction. This difficulty can be avoided by separating from each partial wave an amount equal to the partial wave for pure Coulumb scattering and by summing these pieces to the pseudo closed form discussed below. The scattering amplitudes are then written as

$$
\begin{aligned}
& f(\theta, k)=f_{c}(\theta, k)+f_{N}(\theta, k) \\
& g(\theta, k)=g_{c}(\theta, k)=g_{N}(\theta, k),
\end{aligned}
$$

where $f_{C}$ and $g_{c}$ are the Coulomb amplitudes. $f_{N}$ and $g_{N}$ are the remainders of the scattering amplitudes, which are largely due to the strong interaction and should contain few partial waves.

The Coulomb amplitudes and phase shifts used in SCATPI are

$$
\begin{aligned}
f_{c}^{ \pm} & =\mp(1-t)^{-4} \frac{\alpha}{1-\cos \theta}\left\{\frac{1}{k B} \exp \left(\mp \frac{i \alpha}{6} \ln \frac{1-\cos \theta}{2}\right)\right. \\
& +\frac{1}{2\left(E_{\pi}+E_{p}\right)}\left(\frac{E_{\pi}+E_{p}-M_{N}}{E_{p}-M_{N}}+\frac{E_{\pi}+E_{p}+M_{p}}{E_{p}+M_{p}} \cos \theta-\left(\mu_{p}-1\right) \frac{E_{p}}{M_{p}}(1-\cos \theta)\right. \\
& \left.\left.-\left(\mu_{p}-1\right) \frac{E_{p}-M_{p}}{2 M_{p}} \sin ^{2} \theta\right)-\frac{1}{k \beta}\right\} \\
g_{c}^{ \pm} & = \pm(1-t)^{-4} \frac{\alpha \sin \theta}{2\left(E_{\pi}+E_{p}\right)(1-\cos \theta)}\left(-\frac{E_{\pi}+E_{p}+M_{p}}{E_{p}+M_{p}}+\left(\mu_{p}-1\right) \frac{2 E_{\pi}+E_{p}+M_{p}}{2 M_{p}}\right. \\
& \left.+\left(\mu_{p}-1\right) \frac{E_{p}+M_{p}}{2 M_{p}} \cos \theta\right)
\end{aligned}
$$




$$
\begin{aligned}
& v_{1}^{ \pm}=v_{0}^{ \pm} \pm \frac{\alpha}{\beta} \frac{1}{3 K}\left\{1-(1+K)^{-3}\right\} \\
& v_{2}^{ \pm}=v_{0}^{ \pm} \pm \frac{3 \alpha}{2 \beta} \frac{1}{3 K^{2}}\left\{2 K-1+(1+K)^{-2}\right\} \\
& v_{3}^{ \pm}=v_{1}^{ \pm} \pm \frac{5 \alpha}{6 \beta} \frac{1}{k^{3}}\left\{4-3 K+2 K^{2}-(4+5 K)(1+K)^{-2}\right\},
\end{aligned}
$$

where $f_{c}^{+}, g_{c}^{+}$and $\nu_{i j}^{+}$apply for like charges while $f_{c}^{-}, g_{c}^{-}$and $\nu_{l}^{-}$apply for opposite charges. $f_{c}$ and $g_{c}$ are constructed from the exact nonrelativistic solution for point charges, an additive relativistic correction to first order in $\alpha,{ }^{17}$ the fine structure constant, and $a$ multiplicative form factor $(1-t)^{-4}$, which describes the charge distributions. 11 The $v_{\ell}$ are derived from $f_{c}$ and $g_{c}$ after deleting the magnetic moment terms and ignoring the spin-flip amplitude, both of which are good numerical approximations. ${ }^{11}$ Here $t=\frac{1}{2} k(1-\cos \theta)$ is the square of the momentum transfer, and $k=\left(2 \lambda_{\rho} k\right)^{2}$ with $\lambda_{\rho}=0.2563 \mathrm{fm}$ the Compton wavelength of the $\rho$ meson. $E_{\pi}$ and $E_{p}$ are the total energies of the pion and proton, respectively, in the center of momentum system, while $M_{p}$ is the proton rest energy, all in units of ic. $\mu_{p}$ is the proton magnetic moment in nuclear magnetons. $B C$ is the laboratory ve?ority of the incident pion.

The amplitudes $f_{N}$ and $g_{N}$ can be expanded in partial wave series as

$$
\begin{aligned}
& f_{N}(\theta, k)=\sum_{\ell=0}^{\infty} \exp \left(i \varepsilon v_{\ell}\right)\left\{(\ell+1) A_{\ell+}(k)+\ell A_{\ell-}(k)\right\} P_{\ell}^{0}(\cos \theta), \\
& g_{N}(\theta, k)=\sum_{\ell=0}^{\infty} \exp \left(i \varepsilon v_{\ell}\right)\left\{A_{\ell+}(k)-A_{\ell-}(k)\right\} P_{\ell}^{1}(\cos \theta) .
\end{aligned}
$$

Here $\varepsilon=2$ for eiastic scattering and $\varepsilon=1$ for charge exchange scattering, $A_{\ell \pm}$ are the partial wave amplitudes for total angular momentum 
$j=\ell \pm \frac{1}{2}$, and $P_{\ell}^{0}$ and $P_{\ell}^{l}$ are the associated Legendre functions. Only a few terms contribute to these sums due to the short range of the strong interaction. For energies below about $300 \mathrm{MeV}$, Carter, Bugg and Carter find that only terms with $\ell \leq 3$ are important. For $\pi^{ \pm}$p elastic scattering, SCATPI calculates the differential cross section without Coulomb scattering by using $f_{N}$ and $g_{N}$ as the complete scattering amplitudes, or includes Coulomb scattering by adding $f_{c}$ and $g_{c}$ to these to obtain the complete scattering amplitudes.

The strong interaction between pions and nucleons, which have isospin 1 and $\frac{1}{2}$, respectively, is thought to depend only on the total isospin I, and not on orientation in its space. If this were the only coupling operative, states of total isospin would be eigenstates and scattering amplitudes for the several pion-nucleon charge states would be linear combinations of those amplitudes for $I=\frac{1}{2}$ and $3 / 2$ times the appropriate products of Clebsch-Gordon coefficients. The electromagnetic interaction, however, conserves only $I_{3}$, not $I$, and thus perturbs this simple description. Some of the better understood charge dependent effects are explicitly isolated when the partial wave scattering amplitudes are written in the following form, which was used by Carter, Bugg and Carter.

$$
\begin{aligned}
A_{\ell \pm}\left(\pi^{+} p \rightarrow \pi^{+} p\right) & =\frac{1}{2 i k}\left\{\exp \left(2 i\left(\delta_{3 \ell \pm}+c_{3 \ell \pm}\right)\right)-1\right\} \\
A_{\ell \pm}\left(\pi^{-} p \rightarrow \pi^{-} p\right) & =\frac{1}{2 i k}\left\{\frac{2}{3} \exp \left(2 i\left(\delta_{1 \ell \pm}-\frac{2}{3} c_{1 \ell \pm}\right)\right)\right. \\
& \left.+\frac{1}{3} \exp \left(2 i\left(\delta_{3 \ell \pm}-\frac{1}{3} c_{3 \ell \pm}\right)\right)-1\right\} \\
& -\frac{4}{9 k} c_{13 \ell \pm} \exp \left(i\left(\delta_{1 \ell \pm}+\delta_{3 \ell \pm}\right)\right)
\end{aligned}
$$


Subscripts 1 and 3 refer to isospin $\frac{1}{2}$ and $3 / 2$, respectively. In a potential model, joint action of the Coulomb and strong interaction potentials creates charge dependent phase shifts given by $C_{1 \ell \pm}$ and $C_{3 \ell \pm .} .18$ It also adds charge dependent terms scaled by $c_{13 \ell \pm}$ to the scattering amplitudes with $I_{3}=\frac{1}{2}$, because total isospin is no longer conserved. The remainder of the phase shift $\delta$ is caused mostly by the strong interaction; however, it may still depend on the charge states in several ways. The elasticity, $\exp (-2 \operatorname{Im}(\delta))$, refiects loss to other channels, which may well depend on the charge state. For the energies considered here, elasticities with $I=3 / 2$ differ for $\pi^{+} p$ and $\pi^{-} p$ initial states because a $\gamma n$ final state is available for the $\pi^{-} p$ initial state. The $\operatorname{Re}(\delta)$ may also depend on the charge state through electromagnetic effects which have not been explicitly isolated. Charge dependence in the location and width of resonances in the strong interaction would be an interesting example of such effects.

The phase shifts $\delta$ used in SCATPI are interpolated from those found in the energy independent analysis of Carter, Bugg and Carter, who use the formulation described above. Plausible functions of momentum that have been fitted to the eleven sets of phase shifts for $\pi^{+} p$ and nine sets for $\pi^{-p}$ generate estimates of the phase shifts for all energies between $100 \mathrm{MeV}$ and $300 \mathrm{MeV}$. Proper selection of functions which automatically fulfill known theoretical constraints and contain as few unknown parameters as possible is crucial for efficient use of the experimental informatioll and for physically reliable interpolation. Nearly all contributions to $\delta$ are expected to have short range since the pure Coulomb scattering amplitude has been explicitly isolated and 
removed. To good approximation, therefore, $\delta$ will have the momentum dependence $^{19}$

$$
\tan \delta_{\ell}=k^{2 \ell+1} V\left(k^{2}\right) .
$$

The interpolation functions must have this form. For the $P_{33}$ phase shift, the function includes a polynomial term in addition to a modified Breit-Wigner resonance

$$
\delta=k^{3} \sum_{m=0}^{N} a_{m} k^{2^{m}}+\arctan \frac{\Gamma_{e q}(k)}{2\left(E_{r}-E\right)-i \Gamma_{i n}(k)} .
$$

Here $E$ is the total center of momentum energy, $E_{r}$ is the rest energy of the resonance, and $\Gamma_{e \ell}$ and $\Gamma_{i n}$ are the elastic and inelastic widths of the resonance. The elastic width has the form given by Jackson 20

$$
\Gamma_{e \ell}=\Gamma_{r}\left(\frac{2 E_{r}}{E_{r}+E}\right)\left(\frac{k}{k_{r}}\right)^{3} \frac{1+\left(R k_{r}\right)^{2}}{1+(k k)^{2}}
$$

with $k_{r}$ the wave number at resonance, and $\Gamma_{r}$ and $R$ constants. The inelastic width is approximated as constant for $\pi^{-p}$ and zero for $\pi^{+} p$. The $a_{m}$ are also constants. We wished to model the $P_{11}$ inelasticity as arising through the $N(1470)$ resonance although only the tail of this resonance is in the energy range of interest. Therefore, the function for the $P_{11}$ phase shift also has this form, but with $R=0$ in $\Gamma_{e l}$. Since the inelasticity is believed to be largely in the pion production channel, the inelastic width has the form

$$
\begin{aligned}
\Gamma_{\text {in }} & =0, E<E_{t}, \\
& =b\left(E-E_{t}\right)^{2}, E \geq E_{t},
\end{aligned}
$$

with $E_{t}$ the threshold for pion production and $b$ a constant. For the re- 
maining phase shifts, the functions have the form

$$
\delta_{\ell}=k^{2 \ell+1} \sum_{m=0}^{N} a_{m} k^{2 m} .
$$

In these equations the constants $a_{m}$ carry the same subscripts as $\delta$, so that, for instance $a_{m, 3,1-}$ are associated with $\delta_{3,1-}$, the $P_{31}$ phase shift. Each phase shift was fit to the appropriate form for several trial values of $N$. SCATPI interpolates $\delta_{i}$, $\boldsymbol{i}$ indicating all of the subscripts, using the parameter values obtained with the smallest value of $N_{i}$ for which a reasonable $X^{2} / \nu$ was achieved. Tables $X I X$ and $X X$ list respectively the real and imaginary parts of the $a_{m}$ while the resonance parameters are listed in Table XXI. Following Carter, Bugg and Carter, the imaginary parts of the phase shifts are zero for $\pi^{+} p$. Similarly, the real part of the $P_{33}$ phase shift is also charge dependent. Since only the low energy tail of the $N(1470)$ resonance lies in the energy range of interest, the width and rest energy were taken from the "Review of Particle Properties"21 and not treated as free parameters. $E_{t}$ was assigned the value $6.157 \mathrm{fm}^{-1}$. The $S_{1 i}$ phase shift at $310 \mathrm{MeV}$ precluded

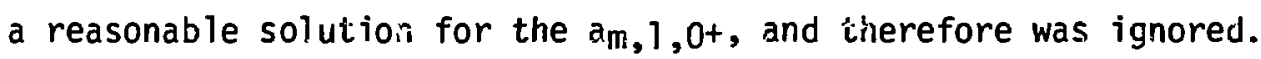

The calculation of $C_{1}, C_{3}$ and $C_{13}$ involves five numerical integrations for each value of $\ell \pm$. For convenience, they are interpolated from the values used by Carter, Bugg and Carter. These quantities depend on the strong as well as the coulomb interaction, and are important only in the first few partial waves. The forms used for their interpolation are

$$
\begin{aligned}
& c_{3,1+}=c_{r 3} \arctan \left(\frac{E-E_{2}}{\Gamma_{1} / 2}\right) /\left\{\left(\frac{E-E_{1}}{\Gamma_{1} / 2}\right)^{2}+\frac{E_{1}-M_{p}-m_{\pi}}{E-M_{p}-m_{\pi}}\right\}, \\
& c_{13,1+}=c_{0}+\frac{c_{r 13}(\Gamma / 2)}{\left(E-E_{1}\right)^{2}+(\Gamma / 2)^{2}}
\end{aligned}
$$


Table XIX. The real part of the polynomial parameters for the phase shifts. The $a_{i}$ are in units of degrees times $f m$ to the appropriate power. $\delta_{1,3+}$ and $\delta_{3,3-}$, not listed, are zero.

\begin{tabular}{cccccc}
\hline $\begin{array}{c}\text { Initial } \\
\text { State }\end{array}$ & $\begin{array}{c}\text { Spectroscopic } \\
\text { Notation }\end{array}$ & $2 I, \ell \pm$ & $\operatorname{Re}\left(a_{0}\right)$ & $\operatorname{Re}\left(a_{1}\right)$ & $\operatorname{Re}\left(a_{2}\right)$ \\
\hline & $S_{11}$ & $1,0+$ & 9.5464 & - & - \\
& $P_{11}$ & $1,1-$ & -6.1009 & 2.5928 & - \\
& $P_{13}$ & $1,1+$ & -2.0870 & 0.4606 & - \\
& $D_{13}$ & $1,2-$ & 0.4706 & - & - \\
& $D_{15}$ & $1,2+$ & 0.7268 & -0.4659 & 0.0925 \\
& $F_{15}$ & $1,3-$ & 0.0704 & -0.0181 & - \\
& $S_{31}$ & $3,0+$ & -6.4878 & -7.5756 & 1.9960 \\
& $P_{31}$ & $3,1-$ & -4.6544 & 1.0635 & - \\
$\pi^{+} p$ & $P_{33}$ & $3,1+$ & 1.3270 & - & - \\
$\pi^{-} p$ & $P_{33}$ & $3,1+$ & -0.2805 & - & - \\
& $D_{33}$ & $3,2-$ & 0.2270 & -0.1247 & 0.0172 \\
& $D_{35}$ & $3,2+$ & -0.5524 & 0.3484 & -0.0701 \\
& $F_{37}$ & $3,3+$ & 0.1334 & -0.0466 & - \\
\hline \hline
\end{tabular}


Table XX. The imaginary part of the polynomial parameters for the phase shifts. Units of $a_{i}$ a re $f m$ to the appropriate power. The parameters not listed are zero.

\begin{tabular}{cccccc}
\hline $\begin{array}{c}\text { Initial } \\
\text { State }\end{array}$ & $\begin{array}{c}\text { Spectroscopic } \\
\text { Notation }\end{array}$ & $21, \ell \pm$ & $\operatorname{Im}\left(a_{0}\right)$ & $\operatorname{Im}\left(a_{1}\right)$ & $\operatorname{Im}\left(a_{2}\right)$ \\
\hline$\pi^{-p}$ & $S_{11}$ & $1,0+$ & 0.2789 & 0.1092 & 0.0148 \\
$\pi-p$ & $S_{11}$ & $3,0+$ & 0.0991 & -0.0523 & 0.0136 \\
\hline
\end{tabular}

Tatle XXI. Resonance parameters for $P_{11}$ and $P_{33}$ phase shifts.

\begin{tabular}{|c|c|c|c|c|c|c|c|}
\hline $\begin{array}{l}\text { Initial } \\
\text { State }\end{array}$ & $\begin{array}{l}\text { Spectrosccpic } \\
\text { Notation }\end{array}$ & $\begin{array}{c}E_{r} \\
2 I, \ell \pm\left(f m^{-1}\right)\end{array}$ & $\begin{array}{l}\Gamma_{r} \\
\left(f m^{-1}\right)\end{array}$ & $\begin{array}{c}R \\
(\mathrm{fm})\end{array}$ & $\begin{array}{l}\Gamma_{\text {in }} \\
\left(\mathrm{fm}^{-1}\right)\end{array}$ & $\begin{array}{l}E_{t} \\
\left(f m^{-1}\right)\end{array}$ & $\begin{array}{c}\text { b } \\
(\mathrm{fm})\end{array}$ \\
\hline$\pi-p$ & $P_{11}$ & $1,1-7.4487^{a}$ & $1.2669^{a}$ & 0.0 & - & $6.1565^{\mathrm{a}}$ & $0.417 t$ \\
\hline$\pi^{-} p$ & $P_{33}$ & $3,1+6.2467$ & 0.6109 & 0.9201 & 0.0 & - & - \\
\hline \multirow[t]{2}{*}{$\pi^{-} p$} & $P_{33}$ & $3,7+6.2422$ & 0.5789 & 1.2290 & $5.69 x$ & - & - \\
\hline & & & & & $10^{-3}$ & & \\
\hline
\end{tabular}

aThese parameters were not varied in minimizing $x^{2} . E_{r}$ and $\Gamma_{r}$ were taken from "Review of Paricicle Properties". 21 
and for the $S$-wave and remaining $P$-wave corrections

$$
c=c_{0}+c_{1} k \text {, }
$$

while the corrections for higher partial waves were ignored. Here $m_{\pi}$ is the rest energy of the pion, $E_{1}, E_{2}, \Gamma_{1}, C_{r}, C_{0}$ and $C_{1}$ are constants. The form of the elastic width in the $P_{11}$ resonance was used for $\Gamma$ here for convenience. The parameter values for each correction were determined by fitting the appropriate form to the values used for that correction by Carter, Bugg and Carter, and are listed in Table XXII.

In Fig. 25 through $2 \varepsilon$ the predictions of SCATPI are compared with the measurements of the differential cross sections by Bussey et al., a subset of the data used in the phase shift analysis of Carter, Bugg and Carter. The agreement with the $\pi^{+} p$ data is excellent, since the deviations are consistent with the accuracy of the measurements. The deviations of the predictions from the $\pi^{-p}$ data below $260 \mathrm{MeV}$ are also consistent with the accuracy of the measurements, but above $260 \mathrm{MeV}$, at the highest two energies, the deviations grow to $3-4 \%$, larger than the supposed uncertainties in the measurements. This trend presumably stems from the difficulty encountered by Carter, Bugg and Carter with the determination of the $I=\frac{1}{2}$ fhase shifts above the $\Delta(1232)$ resonance. Their difficulty may in part be due to the inconsistencies in the data which are suggested in Fig. 28.

\section{Interpolation at $330 \mathrm{MeV}$ and $356 \mathrm{MeV}$}

The differential cross sections at $330 \mathrm{MeV}$ and $356 \mathrm{MeV}$ were interpolated in a graphical manner. The published measurements of the $\pi$-p elastic cross sections above $300 \mathrm{MeV}$ are sparse and plagued with system- 
Table XXII. Parameters for C-quantities.

\begin{tabular}{|c|c|c|c|c|c|}
\hline $\begin{array}{c}\text { Partial } \\
\text { Wave }\end{array}$ & $\ell \pm$ & $\begin{array}{l}c_{3,1+} \\
c_{1}\left({ }^{\circ}\right)\end{array}$ & $\Gamma_{1}\left(\mathrm{fm}^{-1}\right)$ & $E_{1}\left(\mathrm{fm}^{-1}\right)$ & $E_{2}\left(\mathrm{fm}^{-1}\right)$ \\
\hline$P_{3 / 2}$ & $1+$ & 1.0779 & 0.4970 & 0.6238 & 1.0547 \\
\hline $\begin{array}{l}\text { Partial } \\
\text { Wave }\end{array}$ & $\ell \pm$ & $\begin{array}{l}c_{13,1+} \\
c_{0}\left({ }^{\circ}\right)\end{array}$ & $c_{r}\left({ }^{0}\right)$ & $\Gamma_{r}\left(\mathrm{fm}^{-1}\right)$ & $E_{r}\left(\mathrm{fm}^{-1}\right)$ \\
\hline$P_{3 / 2}$ & $1+$ & -0.0607 & -0.3401 & -0.6848 & -0.7315 \\
\hline $\begin{array}{l}\text { Partial } \\
\text { Wave }\end{array}$ & $\ell \pm$ & $\begin{array}{l}\text { C-Quantit } \\
\text { Quantity }\end{array}$ & $c_{0}$ & & $c_{1}\left({ }^{\circ} \mathrm{fm}\right)$ \\
\hline \multirow[t]{3}{*}{$s_{1 / 2}$} & \multirow[t]{3}{*}{$0+$} & $c_{1,0+}$ & \multicolumn{2}{|c|}{-0.1284} & 0.0863 \\
\hline & & $c_{13,0+}$ & \multicolumn{2}{|c|}{0.0185} & 0.0318 \\
\hline & & $c_{3,0+}$ & \multicolumn{2}{|c|}{0.0887} & 0.0569 \\
\hline \multirow[t]{3}{*}{$P_{1 / 2}$} & \multirow[t]{3}{*}{$1-$} & $c_{1,1-}$ & \multicolumn{2}{|c|}{0.1316} & -0.1091 \\
\hline & & $c_{18,1-}$ & \multicolumn{2}{|c|}{-0.0393} & -- \\
\hline & & $c_{3,1-}$ & \multicolumn{2}{|c|}{-0.0282} & $0.079 ?$ \\
\hline$P_{3 / 2}$ & $1+$ & $c_{1,1+}$ & \multicolumn{2}{|c|}{-0.0168} & 0.0434 \\
\hline
\end{tabular}




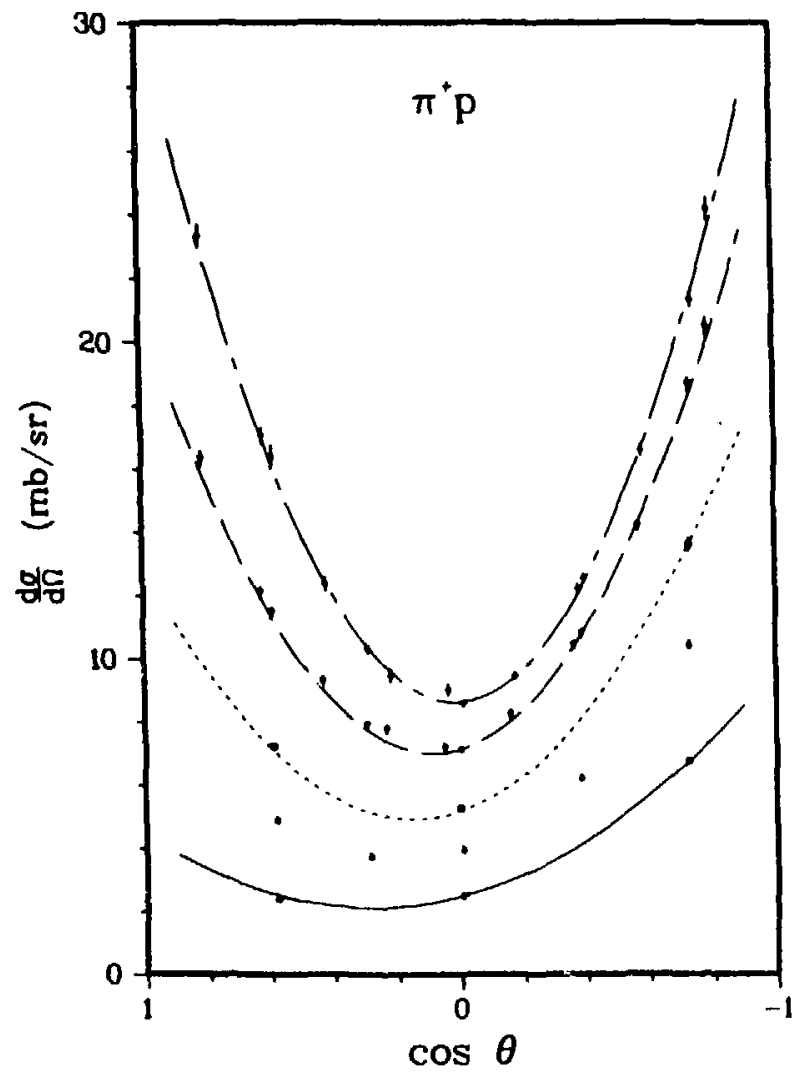

Fig. 25. Angular distributions of Bussey et al. for $\pi^{+} p$ elastic scattering at lab. energies (0) 94.5 , ( $\Delta) 114.1,(\infty) 124.8,(\nabla) 142.9$ and (s) $166.0 \mathrm{MeV}$. The curves show the predictions of SCATPI excluding Coulomb scattering.

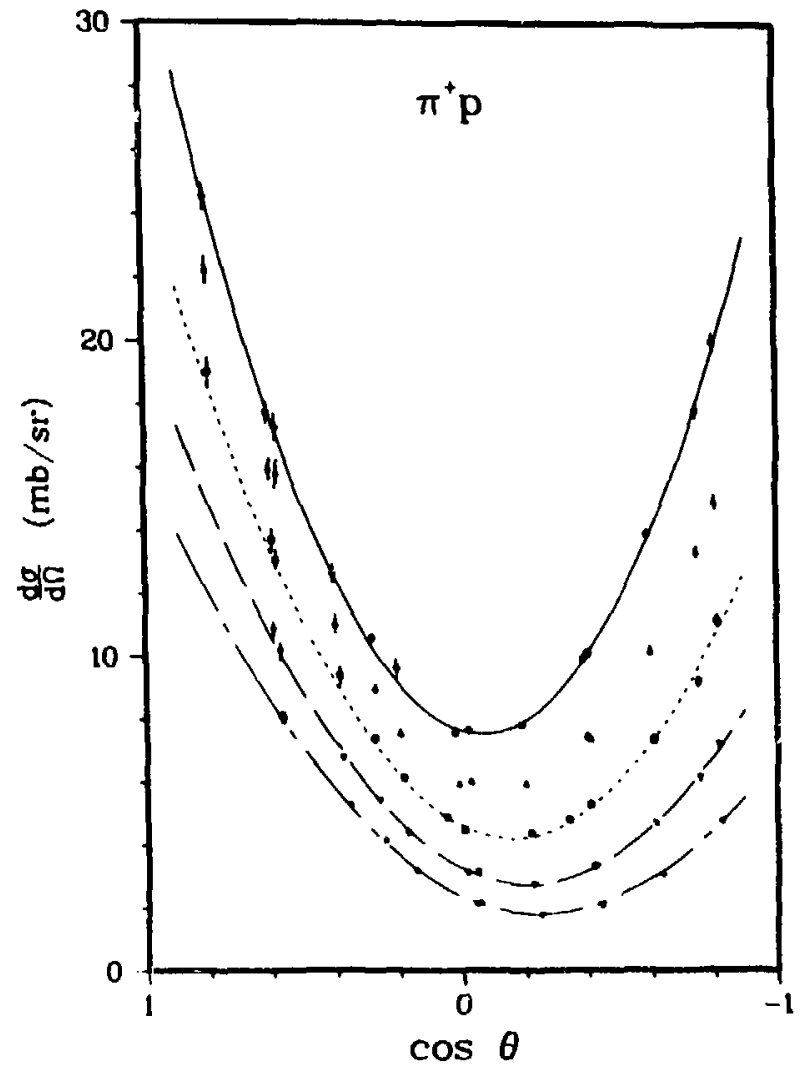

Fig. 26. Angular distributions of Bussey et al. for $\pi^{+} p$ elastic scattering at lab. evergies (0) $194.3,(\Delta) 214.6$, (u) 236.3 , ( $\nabla) 263.7$ and (0) $291.4 \mathrm{MeV}$. The curves show the predictions of SCATPI excluding Coulomb scattering. 
$\infty$

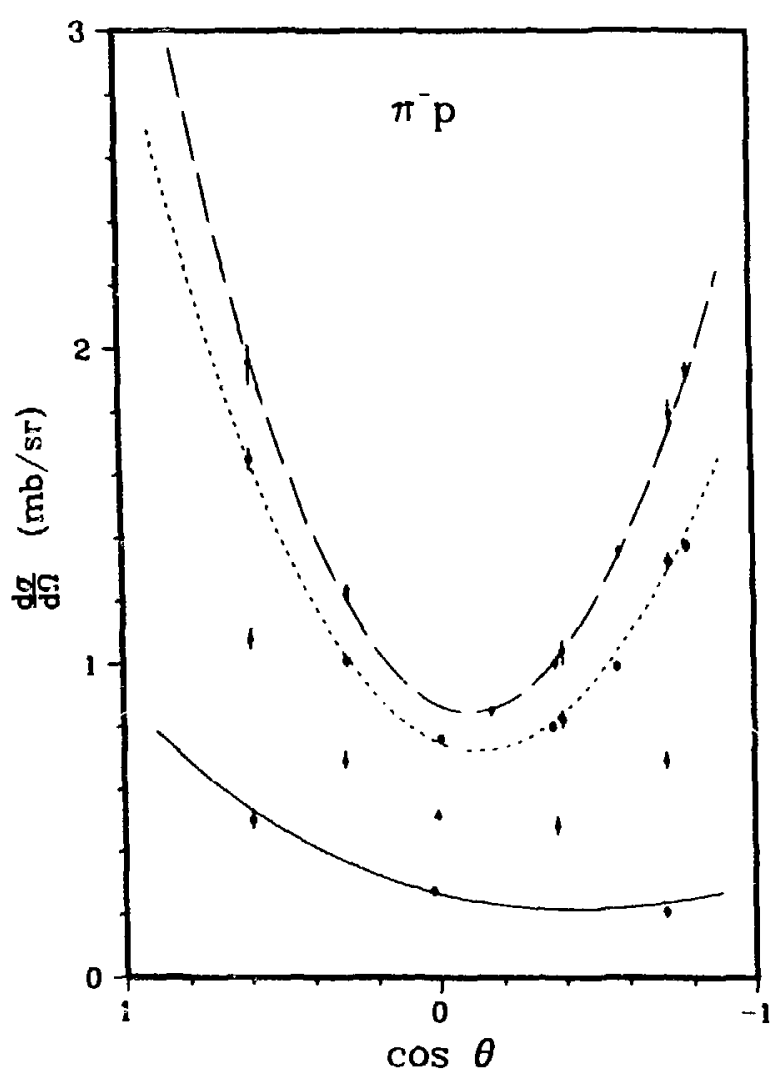

Fig. 2?. Angular distributions of Bussey et al. for $\pi^{-p}$ elastic scattering at lab. energies (0) 88.5 , $(\Delta) 119.3,(0) 144.2$ and $(\nabla) 161.9 \mathrm{MeV}$. The curves show the predictions of SCATPI excluding Coulomb scattering.

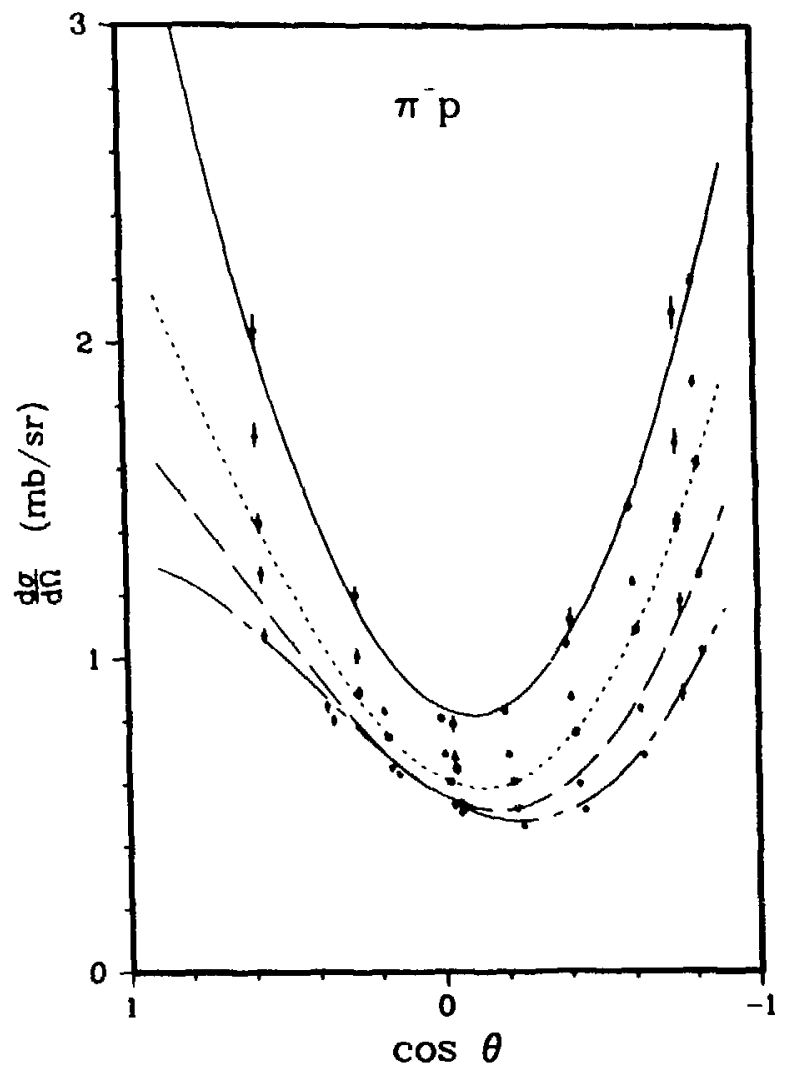

Fig. 28. Angular distributions of Bussey et al. for $\pi^{-p}$ elastic scattering at lab. energies (0) $191.9,(\Delta) 219.6$, (ㄷ) $237.9,(\nabla) 263.7$ and $(\diamond) 291.5 \mathrm{MeV}$. The curves show the predictions of SCATPI excluding coulomb scattering. 
atic errors. The most reliable appear to be those of ogden et a1.22 at $370 \mathrm{MeV}$ and $410 \mathrm{MeV}$, and Rugge and $\mathrm{Vik}^{23}$ at $310 \mathrm{MeV}$. The cross sections at the two highest energies were interpolated from these measurements and the measurements of Bussey et a 7.12 at $264 \mathrm{MeV}$ and $292 \mathrm{MeV}$.

The data were plotted as a function of the cosine of the center of momentum scattering angle, $\cos \theta$, and a curve was sketched through each of the five sets of data. The cross sectioris and curve for $370 \mathrm{MeV}$ are shown in Fig. 29.as an example. The cross sections for a particular $\cos \theta$ were interpolated at each energy using these curves. Uncertainties were assigned to the interpolated cross sections on the basis of the uncertainties of nearby measurements. The cross sections for $\cos \theta$ were plotted as a function of the incident kinetic energy. A curve was sketched through these points, bearing in mind that the form of the curve should change smoothly and systematically with $\cos \theta$. Fig. 30 shows the cross section as a function of incident kinetic energy for five values of $\cos \theta$ as an example. The required values of the differential cross section were interpolated from these curves. Rough errors were assigned on the basis of reasonable distortions in the curves. 


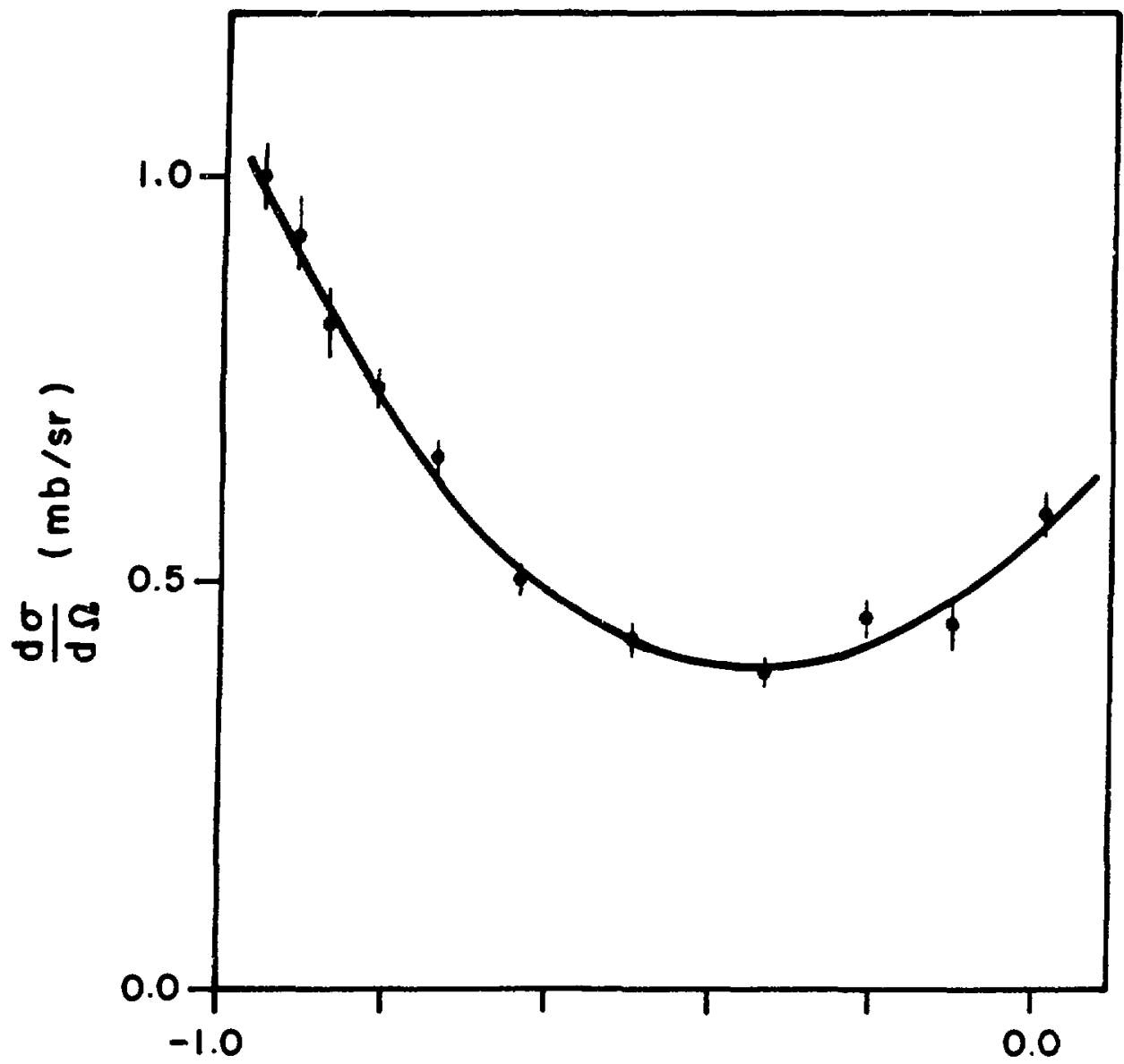

$\cos \theta$

Fig. 29. Angular distribution of Ogden et al. at $370 \mathrm{MeV}$. The curve was sketched through the points to aid in interpolation. 


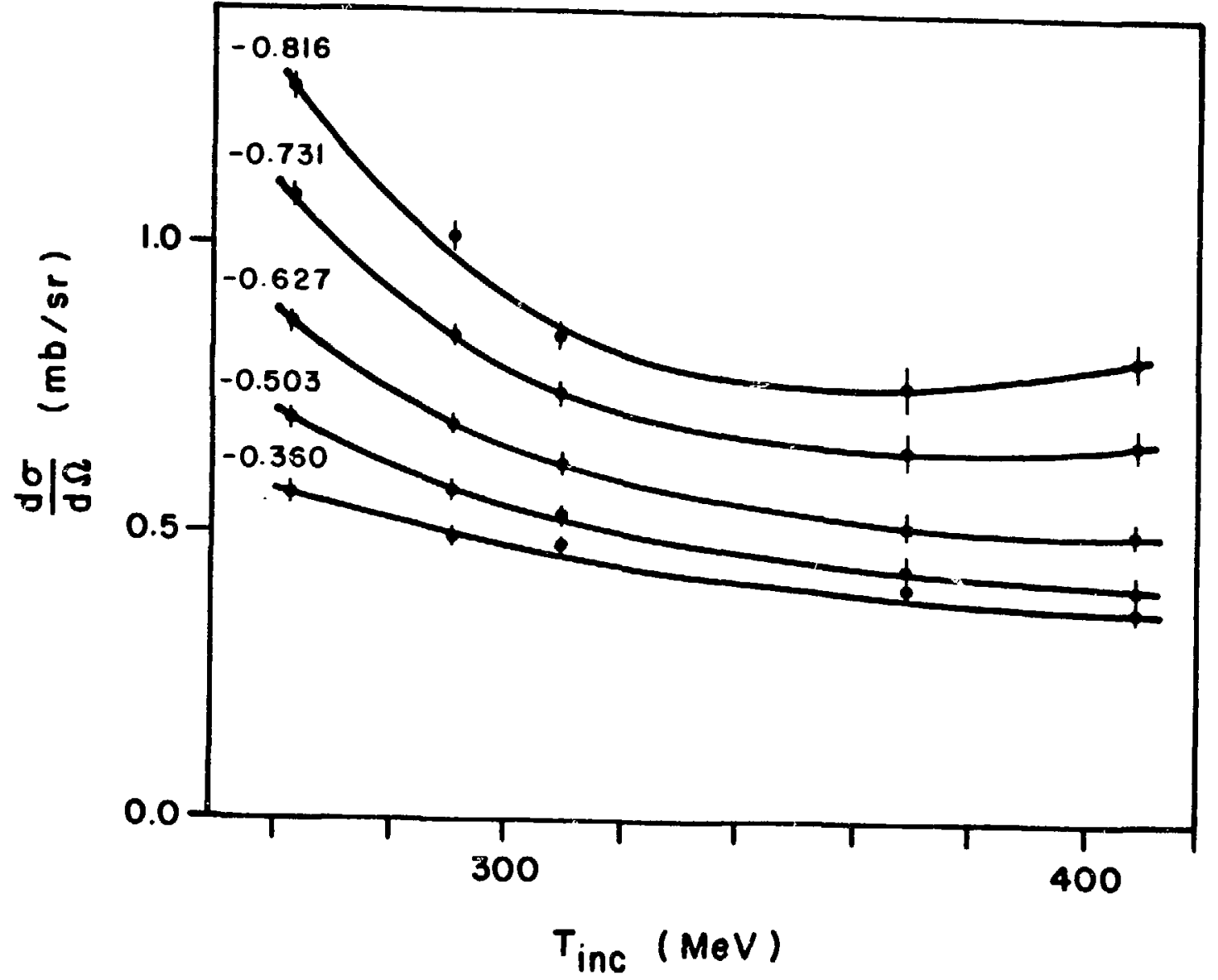

Fig. 30. Variation of the differential cross section with incident energy. The curves were $\stackrel{\infty}{\omega}$ sketched through the points to aid in interpolation. 


\section{CHAPTER $V$}

\section{ANALYSIS OF $\pi^{+}$PRODUCTION}

The doubly differential cross section $\frac{d^{2} \sigma}{d \Omega d T}$ of the $\pi^{+}$meson produced in the reaction $\pi^{-} p \rightarrow \pi^{+} \pi^{-} n$ was measured at six incident energies between $229 \mathrm{MeV}$ and $356 \mathrm{MeV}$. Plausible functions of the kinetic energy $\mathrm{T}$ and the cosine of the angle $\theta$ in the center of momentum frame were fitted to measurements at each energy. The functions were integrated to obtain the average square midulus of the matrix element and the net cross section for the reaction. The mean square moduli were extrapolated to the $172 \mathrm{MeV}$ threshuld to determine the symmetry breaking parameter of soft pion theory.

\section{Doubly Differential Cross Sections}

Event raie. Each measurement of $\frac{d^{2} \sigma}{d \Omega d T}$ consisted of two sets of data, one for the target flask filled with liquid hydrogen and one for the flask emptied. The doubly differential cross section was calculated from the difference in rates for the two sets. This procedure does not correct for the $\mathrm{e}^{+}$background which results from the sequence of single charge exchange in the target and flask, $\pi^{0}$ decay and pair production in the spectrometer. Since this rate also depends on the status of the target, it must be eliminated in another fashion. The detected $e^{+}$are transported most of the way by the spectrometer, and hence are energetic and can be identified by a Cerenkov detector. The efficiency $\varepsilon$ for identifying these $e^{+}$is critical, particularly at $229 \mathrm{MeV}$ where the charge exchange cross section is $\sim 30 \mathrm{mb}$ while the 
$\pi^{+}$production cross section is only $\sim 60 \mu \mathrm{b}$. A recent measurement ${ }^{3}$ of the $\pi^{+}$production cross section at $230 \mathrm{MeV}$ leaves no doubt that $\varepsilon$ was significantly less than unity. Appendix $C$ describes the evaluation of $\varepsilon$ for most of the data from the measurements at $229 \mathrm{MeV}$ and $230 \mathrm{MeV}$, with the result $\varepsilon=0.828 \pm 0.038$. Also described is the determination of $\varepsilon_{e}$, the Cerenkov efficiency for the early data at $292 \mathrm{MeV}$, with the result $\varepsilon_{e}=0.653 \pm 0.28$.

After correcting for the Cerenkov efficiency, the number of events in a channel was

$$
N_{i}=n_{1} i-\frac{1-\varepsilon}{\varepsilon} n_{2 i}
$$

where $n_{1 j}$ is the number of events for which only channel $i$ detected $a$ particle and there was no coincident Cerenkov puise, $n_{2} j$ is the same except there was a coincident Cerenkov pulse. The $\eta_{j i}$ possessed Poisson distributions since the events occurred randomly in time. The statistical uncertainty in the number of events was

$$
\Delta N_{j}=\left\{\left(r_{1 j}+1\right)+\left(\frac{l-\varepsilon}{\varepsilon}\right)^{2}\left(n_{2 j}+1\right)\right\}^{\frac{1}{2}} .
$$

The net rate for $\pi^{+}$events was

$$
R_{i}=\left(N_{i} / M_{\ell}\right)_{\text {full }}-\left(N_{i} / M_{\ell}\right)_{\text {empty }}
$$

with statistical uncertainty $\Delta R_{i}=\left\{\left(\Delta N_{i} / M_{\ell}\right)_{\text {full }}^{2}+\left(\Delta N_{i} / M_{\ell}\right)_{\text {empty }}^{2}\right\}^{\frac{1}{2}}$, where the subscripts full and empty refer to the target status. Here, as before, $M_{\ell}$ is either the number of counts in the scattering monitor during live time, or, for angles forwards of $40^{\circ}$ in the laboratory, the number of counts corrected for the reduction in the effectiveness of the scattering monitor due to the $\pi^{-}$beam intercepting the spectrometer structure. $M_{\ell}$ was sufficiertly large $\left(>10^{4}\right)$ that its uncertainty con- 
tributed negligibly to $\Delta R_{i}$.

Matrix element. Due to conservation of energy there was a maximum momentum with which the $\pi^{+}$(or $\pi^{-}$or $n$ ) could exit the target. Near this maximum the rate falls rapidly, going to zero at the maximum momentum. Here averaging the laboratory rates $R_{j}$ becomes less meaningful. The question was further complicated by the $4 \%$ width of the incident momentum distribution, since the maximum outgoing momentum depends on the total energy of the system.

This problem was resolved by considering the center of momentum cross section $\frac{d^{2} \sigma}{d \Omega d T}$ in terms of the squared modulus of a matrix element $M$ weighted by the density of phase space and averaged over the unobserved kinematic variables

$$
\frac{d^{2} \sigma}{d \Omega d T}=\left\langle|M|^{2}\right\rangle \frac{d^{2} \sigma}{d \Omega d T}
$$

with

$$
\frac{d^{2} \sigma_{0}}{d \Omega d T}=\frac{1}{4 \pi} \frac{\hbar^{2}}{4}\left(\frac{G^{2}}{4 \pi}\right)^{3}\left(\frac{E v}{g_{d}}\right)^{4} \frac{m_{\pi^{2}}}{M_{N}^{4}} \frac{Q}{S}\left(T_{2} \max ^{-T_{2} \min }\right) .
$$

Here $2 \pi \lambda$ is the Compton wavelength of the charged pion, $G$ is the pionnucleon strong coupling constant, $g_{v}$ and $g_{a}$ are the vector and axial vector coupling constants, and $M_{N}$ is the nucleon mass. $Q$ is the incident momentum in the center of momentum system, $S$ is the root of the invariant mass squared, and $T_{2}$ is the kinetic energy of either of the undetected particles in the center of momentum. The last factor was calculated as

$$
T_{2 \max }-T_{2 \min }=\frac{2 p\left[S\left(T_{\max }-T\right)\left(2 m_{2 m}+S\left(T_{\max }-T\right)\right)\right\}^{\frac{1}{2}}}{\left(m_{2}+m_{3}\right)^{2}+2 S\left(T_{\max }-T\right)}
$$

where $p$ and $T$ are the momentum and kinetic energy in the center of mo- 
mentum, $T_{\max }$ is the maximum value of $T$, and $m_{2}$ and $m_{3}$ are the masses of the two undetected particles. $\sigma_{0}$ has been referred to as $Q^{2} \times$ phase space because it includes a factor of $Q^{2}$ from the square modulus of the matrix element which is introduced by the $\mathrm{P}$-wave nature of the initial state. $\sigma_{0}$ also includes flux factors and some constants to facilitate comparison with soft pion theory.

Both the mean square modulus and $Q^{2} \times$ phase space depended on the total energy. Hence, the observed rate was an integral over $w\left(Q_{L A B}\right)$, the distribution of momenta in the beam,

$$
R_{i}=\frac{\eta_{i} P_{C} \delta}{E_{i} T} \int J_{i}<|M|^{2}>\frac{d^{2} \sigma_{Q}}{d \Omega d T} w\left(Q_{L A B}\right) d Q_{L A B} .
$$

Here $n_{j}$ is the channal efficiency, $P_{c} \delta$ is the momentum acceptance of a channel, and $T$ is the normalization factor. $E_{j}$ is the decay correction

$$
E_{i}=\exp \left\{\Lambda m_{\pi} /\left(\tau p_{j}\right)\right\}
$$

with $\Lambda$ the mean path length to the focal plane, $m_{\pi}$ and $\tau$ the rest mass and lifetime, respectively, of the charged pion, and $p_{i}$ the momenta of particles detected in the $i^{\text {th }}$ channel. $J_{j}$ is the Jacobian of the transformation

$$
J_{i}=\frac{\partial(T, \cos \theta)}{\partial\left(P_{L A B}, \cos \theta_{L A B}\right)}=\left(p_{i}^{2}+m_{\pi}^{2}\right)^{-\frac{1}{2}} p_{j} / p
$$

with $p$ the momentum $p_{j}$ transformed to the center of momentum frame. The distribution w was approximated as Gaussian, and the integration was carried out from two standard deviations below the mean to two standard deviations above the mean. w was normalized such that

$$
\int w(x) d x=1
$$

for the same limits of integration. The square modulus of the matrix element weighted and averaged over the unobserved kinematic variables 
and the distribution of the incident momenta was determined from the rate

$$
\left.\left\langle|M|^{2}\right\rangle_{B E A M}\right\rangle_{i}=\frac{T E_{i} R_{i}}{\eta_{i} P_{c} \delta}\left\{\int J_{i} \frac{d^{2} \sigma_{Q}}{d \Omega d T} w\left(Q_{L A B}\right) d Q_{L A B}\right\}^{-1},
$$

with $\left.\Delta M_{i}=\left\langle|M|^{2}\right\rangle_{B E A M}\right\rangle_{j}\left(\Delta R_{j} / R_{j}\right)$ the statistical uncertainty. Here, as above, $\frac{d^{2} \sigma_{0}}{d \Omega d T}$ depends on $i$ as well as $Q_{L A B}$ through the variables $T$ and $\cos \theta$. The mean square modulus is more slowly varying than the cross section near threshold. The estimates from the different channels are averaged to obtain a single estimate for the measurement

with

$$
\left.\left\langle|M|^{2}\right\rangle=\sum_{i} \Delta_{M i}^{-2}\left\langle|M|^{2}\right\rangle_{B E A M}\right)_{j} /\left\{\sum_{j} \Delta_{M j}^{-2}\right\}
$$

$$
\Delta_{M}=\left\{\sum_{j} \Delta_{M j}^{-2}\right\}^{-\frac{1}{2}}
$$

the statistical uncertainty.

Doubly differential cross section. The incident and detected pion momenta associated with the measurement were the mean values of the pions contributing to the measurement. In the approximation that $\left\langle|M|^{2}\right\rangle$ is slowly varying as a function of the incident and detected momenta, this implies

$$
\begin{aligned}
\left\langle Q_{L A B}\right\rangle=\sum_{i} n_{j} E_{i}^{-1} \int Q_{L A B} j_{i} \frac{d^{2} \sigma_{0}}{d \Omega d T} w\left(Q_{L A B}\right) d Q_{L A B} \\
\quad \times\left\{\sum_{j} \eta_{j} E_{j}^{-1} \int j_{j} \frac{d^{2} \sigma_{0}}{d \Omega d T} w\left(Q_{L A B}\right) d Q_{L A B}\right\}^{-1}
\end{aligned}
$$

and

$$
\begin{aligned}
\left\langle P_{L A B}\right\rangle= & \sum_{i} \eta_{i} E_{i}^{-1} p_{j} \int j_{i} \frac{d^{2} \sigma_{0}}{d \Omega d T} w\left(Q_{L A B}\right) d Q_{L A B} \\
& \times\left\{\sum_{j} \eta_{j} E_{j}^{-1} \int J_{j} \frac{d^{2} \sigma_{0}}{d \Omega d T} w\left(Q_{L A B}\right) d Q_{L A B}\right\}^{-1}
\end{aligned}
$$

where $\left\langle|M|^{2}>\right.$ has been factored from each of the integrals, and $\left\langle Q_{L A B}>\right.$ and $\angle P_{L A B}>$ are the mean incident and detected momentum, respectivelý. The cross section in the center of momentum frame was calculated from 
$\left\langle|M|^{2}\right\rangle$ with $\frac{d^{2} \sigma_{0}}{d z d}$ evaluated for the mean momenta.

Tables XXIII through XXVII present $T, \cos \theta, \frac{d^{2} \sigma_{0}}{d \Omega d T},\left\langle|M|^{2}\right\rangle$ and the percent uncertainties in $\left\langle|M|^{2}\right\rangle$ for the measurements at the higher five incident energies. These percent uncertainties apply to the doubly differential cross section as well. The uncertainties $\Delta_{M \varepsilon}$ are the deviations in $\left\langle|M|^{2}\right\rangle$ produced by the substitution of $\varepsilon \pm \Delta \varepsilon$ for $\varepsilon$. Presumably, these are highly correlated from measurement to measurement and from energy to energy. The values for $\left\langle|M|^{2}\right\rangle$ and their statistical uncertainties are also shown in Fig. 31 through 35 . The solid and dashed lines represent respectively the laboratory momentum of $70 \mathrm{MeV} / \mathrm{C}$ and the laboratory angle of $32^{\circ}$. The coverage of $(T, \cos \theta)$ space varied from about $65 \%$ at $254 \mathrm{MeV}$ to $85 \%$ at $356 \mathrm{Mel}$.

\section{Integrated Cross Sections}

At each energy the values of $\left\langle|M|^{2}\right\rangle$ were fitted to the function

$$
f(T, \cos \theta)=C(T)\left\{\zeta_{1}+\zeta_{2} \cos \theta+\zeta_{3}\left(T_{\max }-T\right) \cos \theta+\zeta_{4} T^{2}\right\}^{2}
$$

with $\zeta_{j}$ the adjustable parameters. $C(T)$ is a correction for enhancement of the cross section from Coulomb attraction between the two pions in the final state averaged over the unobserved kinematic variables. It was calculated by a Monte Carlo computer program as

$$
C(T)=\left\langle\exp \left(\frac{\pi \alpha}{B}\right)\right\rangle \text {, }
$$

where $\beta C$ is the relative velocity of the two pions and $\alpha$ is the finest. ucture constant. The exponential was averaged over $\dot{\beta}$ for $T$ in sixteen bins between 0 and $T_{\max }$. These values were slowly varying between 1.03 and 1.05 . $C(T)$ was obtained by linear interpolation. The parameters $\zeta_{i}$ were varied to minimize $x^{2}$ 
Table XXIII. Doubly differential cross sections at $254 \mathrm{MeV} . \mathrm{T}$ and $\theta$ are the kinetic energy and angle of the produced $\pi^{+}, \Delta_{1}$ is the percent statistical uncertainty and $\Delta_{2}=\left\{\frac{d}{d \varepsilon}\left\langle\left. M\right|^{2}>\right\} \delta \varepsilon /|M|^{2}\right.$ is the sensitivity to the Cerenkov efficiency $\varepsilon, T$ is in MeV, $\left(\mathrm{d}^{2} \sigma / \mathrm{d} \Omega \mathrm{dT}\right)$ is in $\mu \mathrm{b} /(\mathrm{sr}-\mathrm{MeV})$, and both $\Delta_{1}$ and $\Delta_{2}$ are in $\%$.

\begin{tabular}{|c|c|c|c|c|c|}
\hline$T$ & $\cos \theta$ & $\frac{d^{2} \sigma}{d \Omega d T}$ & $\left\langle\left. M\right|^{2}\right\rangle$ & $\Delta_{1}$ & $\Delta_{2}$ \\
\hline 36.0 & -0.751 & 0.303 & 8.19 & 30.1 & 0.9 \\
\hline 46.2 & -0.751 & 0.148 & 5.23 & 38.6 & 11.1 \\
\hline 25.8 & -0.351 & 0.344 & 8.86 & 19.7 & 8.4 \\
\hline 36.0 & -0.352 & 0.332 & 8.98 & 13.3 & 5.8 \\
\hline 44.5 & -0.354 & 0.195 & 6.44 & 18.9 & 5.8 \\
\hline 15.3 & -0.198 & 0.227 & 6.56 & 32.6 & 2.7 \\
\hline 24.2 & -0.007 & 0.361 & 9.37 & 11.8 & 8.0 \\
\hline 34.3 & -0.005 & 0.371 & 9.84 & 9.9 & 5.8 \\
\hline 43.8 & -0.009 & 0.189 & 6.08 & 15.4 & 8.2 \\
\hline 15.4 & 0.149 & 0.333 & 9.59 & 14.9 & 16.2 \\
\hline 23.7 & 0.294 & 0.296 & 7.68 & 14.2 & 14.4 \\
\hline 34.3 & 0.294 & 0.355 & 9.41 & 11.3 & 7.0 \\
\hline 43.5 & 0.291 & 0.324 & 10.28 & 8.7 & 6.3 \\
\hline 8.6 & 0.349 & 0.357 & 12.87 & 15.1 & 2.1 \\
\hline 15.4 & 0.549 & 0.233 & 8.17 & 14.3 & 22.3 \\
\hline 23.6 & 0.644 & 0.353 & 9.16 & 10.9 & 11.6 \\
\hline 33.6 & 0.644 & 0.356 & 9.36 & 9.0 & 9.4 \\
\hline 42.5 & 0.648 & 0.269 & 8.25 & 10.1 & 10.9 \\
\hline
\end{tabular}


Table XXIV. Doubly differential cross sections at $279 \mathrm{MeV}$. Entries are the same as in Table XxIII.

\begin{tabular}{|c|c|c|c|c|c|}
\hline$T$ & $\cos \theta$ & $\frac{d^{2} \sigma}{d \Omega d T}$ & $\left\langle|M|^{2}\right\rangle$ & $\Delta_{1}$ & $\Delta_{2}$ \\
\hline 47.0 & -0.850 & 0.391 & 7.71 & 22.9 & 4.1 \\
\hline 60.3 & -0.851 & 0.292 & 7.77 & 21.7 & 3.5 \\
\hline 33.6 & -0.790 & 0.476 & 8.91 & 29.0 & 0.3 \\
\hline 34.3 & -0.502 & 0.618 & 11.55 & 11 & 1.6 \\
\hline 45.9 & -0.527 & 0.589 & 11.48 & 10.8 & 0.8 \\
\hline 57.7 & -0.526 & 0.362 & 8.79 & 13.5 & 1.4 \\
\hline 20.2 & -0.451 & 0.395 & 8.30 & 30.6 & 0.7 \\
\hline 15.8 & -0.102 & 0.415 & 9.51 & 16.5 & 0.8 \\
\hline 29.0 & -0.096 & 0.534 & 10.20 & 11.6 & 2.0 \\
\hline 42.7 & -0.106 & 0.545 & 10.36 & 9.5 & 1.2 \\
\hline 55.8 & -0.108 & 0.473 & 10.86 & 9.9 & 0.8 \\
\hline 15.7 & 0.248 & 0.425 & 9.76 & 14.0 & 5.6 \\
\hline 29.1 & 0.291 & 0.655 & 12.49 & 10.1 & 1.9 \\
\hline 42.2 & 0.299 & 0.651 & 12.34 & 8.1 & 1.2 \\
\hline 55.4 & 0.296 & 0.481 & 10.90 & 8.5 & 1.5 \\
\hline 6.7 & 0.349 & 0.218 & 7.17 & 27.5 & 2.9 \\
\hline 15.6 & 0.548 & 0.410 & 9.42 & 16.3 & 6.7 \\
\hline 29.0 & 0.649 & 0.553 & 10.55 & 8.4 & 3.4 \\
\hline 41.8 & 0.649 & 0.661 & 12.50 & 6.8 & 1.8 \\
\hline 52.7 & 0.688 & 0.508 & 10.85 & 7.3 & 2.1 \\
\hline
\end{tabular}


Table XXV. Doubly differential cross sections at $292 \mathrm{MeV}$. Entries are the same as in Table XXIII.

\begin{tabular}{|c|c|c|c|c|c|}
\hline$T$ & $\cos \theta$ & $\frac{d^{2} \sigma}{d \Omega d T}$ & $\left\langle|M|^{2}\right\rangle$ & $\Delta_{1}$ & $\Delta_{2}$ \\
\hline 40.5 & -0.847 & 0.562 & 9.16 & 24.5 & $5.7^{\mathrm{a}}$ \\
\hline 56.7 & -0.848 & 0.605 & 10.99 & 14.6 & $18.1^{\mathrm{a}}$ \\
\hline 67.9 & -0.851 & 0.289 & 6.98 & 25.2 & $24.0^{\mathrm{a}}$ \\
\hline 40.7 & -0.543 & 0.783 & 12.78 & 11.4 & $3.0^{\mathrm{a}}$ \\
\hline 57.0 & -0.544 & 0.601 & 10.95 & 10.0 & $9.0^{a}$ \\
\hline 65.2 & -0.556 & 0.423 & 9.24 & 11.6 & $13.5^{\mathrm{a}}$ \\
\hline 24.4 & -0.439 & 0.657 & 11.77 & 14.5 & $13.7^{\mathrm{a}}$ \\
\hline 24.6 & -0.138 & 0.609 & 10.90 & 12.3 & $22.8^{a}$ \\
\hline 40.9 & -0.138 & 0.764 & 12.47 & 9.2 & $10.0^{\mathrm{a}}$ \\
\hline 57.5 & -0.146 & 0.477 & 8.75 & 11.0 & $9.6^{a}$ \\
\hline 67.5 & -0.153 & 0.403 & 9.48 & 9.3 & $9.6^{\mathrm{a}}$ \\
\hline 25.0 & 0.256 & 0.817 & 14.55 & 8.6 & $20.7^{\mathrm{a}}$ \\
\hline 41.1 & 0.264 & 0.813 & 13.26 & 7.4 & $11.0^{\mathrm{a}}$ \\
\hline 57.7 & 0.254 & 0.562 & 10.33 & 6.6 & $12.4^{\mathrm{a}}$ \\
\hline 67.5 & 0.244 & 0.366 & 8.91 & 7.9 & 1.6 \\
\hline 8.4 & 0.369 & 0.394 & 16.84 & 19.8 & $45.0^{\mathrm{a}}$ \\
\hline 25.1 & 0.559 & 0.737 & 13.10 & 8.2 & 2.1 \\
\hline 41.1 & 0.654 & 0.685 & 11.18 & 7.9 & 1.5 \\
\hline 58.0 & 0.655 & 0.558 & 10.34 & 8.7 & 1.7 \\
\hline 67.2 & 0.649 & 0.352 & 8.19 & 9.5 & 2.2 \\
\hline
\end{tabular}

${ }^{\text {a }}$ Represents the sensitivity to $\varepsilon_{e}$ rather than $\varepsilon$. The sensitivity to $\varepsilon$ is smaller by a factor of 10 . 
Table XXVI. Doubly differential cross sections at $330 \mathrm{MeV}$. Entries are the same as in Table XXIII.

\begin{tabular}{|c|c|c|c|c|c|}
\hline$T$ & $\cos \theta$ & $\frac{d^{2} \sigma}{d \Omega d T}$ & $\left\langle|M|^{2} ?\right.$ & $\Delta_{\eta}$ & $\Delta_{2}$ \\
\hline 48.7 & -0.851 & 0.995 & 11.57 & 10.1 & 0.2 \\
\hline 68.2 & -0.851 & 1.013 & 12.30 & 14.0 & 0.5 \\
\hline 87.4 & -0.851 & 0.713 & 11.89 & 14.2 & 0.5 \\
\hline 25.5 & -0.496 & 0.848 & 17.71 & 11.1 & 0.1 \\
\hline 45.5 & -0.506 & 1.205 & 14.10 & 12.3 & 0.4 \\
\hline 67.7 & -0.501 & 1.053 & 12.52 & 10.4 & 0.3 \\
\hline 84.4 & -0.535 & 0.701 & 10.74 & 11.8 & 0.1 \\
\hline 27.5 & -0.121 & 1.265 & 16.98 & 9.9 & 0.7 \\
\hline 46.6 & -0.120 & 1.174 & 13.68 & 9.5 & 0.4 \\
\hline 65.8 & -0.120 & 1.258 & 15.03 & 7.4 & 0.3 \\
\hline 83.1 & -0.122 & 0.739 & 10.97 & 9.3 & 0.2 \\
\hline 9.7 & -0.054 & 0.573 & 11.98 & 12.7 & 0.5 \\
\hline 27.2 & 0.245 & 1.320 & 17.79 & 7.3 & 0.7 \\
\hline 46.6 & 0.270 & 1.437 & 16.75 & 6.2 & 0.4 \\
\hline 65.3 & 0.276 & 1.215 & 14.48 & 5.6 & 0.4 \\
\hline 82.7 & 0.276 & 0.695 & 10.21 & 7.5 & 0.4 \\
\hline 9.7 & 0.347 & 0.870 & 18.17 & 10.9 & 0.2 \\
\hline 25.7 & 0.553 & 1.257 & 17.29 & 7.6 & 1.3 \\
\hline 45.0 & 0.647 & 1.318 & 15.45 & 5.4 & 0.8 \\
\hline 64.3 & 0.650 & 1.144 & 13.56 & 6.6 & 0.8 \\
\hline 82.7 & 0.653 & 0.685 & 10.06 & 6.3 & 0.7 \\
\hline
\end{tabular}


Table XXVII. Doubly differential cross sections at $356 \mathrm{MeV}$. Entries are the same as in Table XXIII.

\begin{tabular}{|c|c|c|c|c|c|}
\hline$T$ & $\cos \theta$ & $\frac{d^{2} \sigma}{d \Omega d T}$ & $\left\langle|M|^{2}\right\rangle$ & $\Delta_{1}$ & $\Delta_{2}$ \\
\hline 56.0 & -0.851 & 1.389 & 13.43 & 11.2 & 0.0 \\
\hline 78.1 & -0.851 & 1.425 & 14.38 & 10.2 & 0.3 \\
\hline 100.1 & -0.851 & 0.807 & 11.23 & 12.2 & 0.1 \\
\hline 33.6 & -0.552 & 1.277 & 14.03 & 11.5 & 0.1 \\
\hline 55.8 & -0.552 & 1.691 & 16.36 & 8.5 & 0.3 \\
\hline 76.5 & -0.532 & 1.353 & 13.53 & 7.7 & 0.1 \\
\hline 97.6 & -0.523 & 0.857 & 11.18 & 8.6 & 0.1 \\
\hline 11.2 & -0.152 & 0.853 & 14.93 & 14.7 & 0.2 \\
\hline 32.3 & -0.153 & 1.654 & 18.41 & 7.5 & 0.4 \\
\hline 52.4 & -0.143 & 1.628 & 15.87 & 6.9 & 0.3 \\
\hline 74.3 & -0.134 & 1.531 & 15.14 & 5.9 & 0.1 \\
\hline 94.0 & -0.109 & 0.991 & 11.97 & 6.8 & 0.2 \\
\hline 11.2 & 0.247 & 1.266 & 22.19 & 11.3 & 0.2 \\
\hline 31.9 & 0.252 & 1.840 & 20.59 & 6.4 & 0.4 \\
\hline 52.5 & 0.234 & 1.764 & 17.19 & 5.1 & 0.3 \\
\hline 73.0 & 0.270 & 1.652 & 16.24 & 4.4 & 0.2 \\
\hline 93.6 & 0.291 & 0.970 & 11.62 & 5.7 & 0.2 \\
\hline 29.5 & 0.588 & 2.228 & 25.64 & 4.6 & 0.5 \\
\hline 51.0 & 0.636 & 1.969 & 19.28 & 4.7 & 0.5 \\
\hline 72.3 & 0.656 & 1.616 & 15.84 & 4.6 & 0.3 \\
\hline 93.2 & 0.668 & 0.956 & 11.37 & 5.2 & 0.7 \\
\hline
\end{tabular}




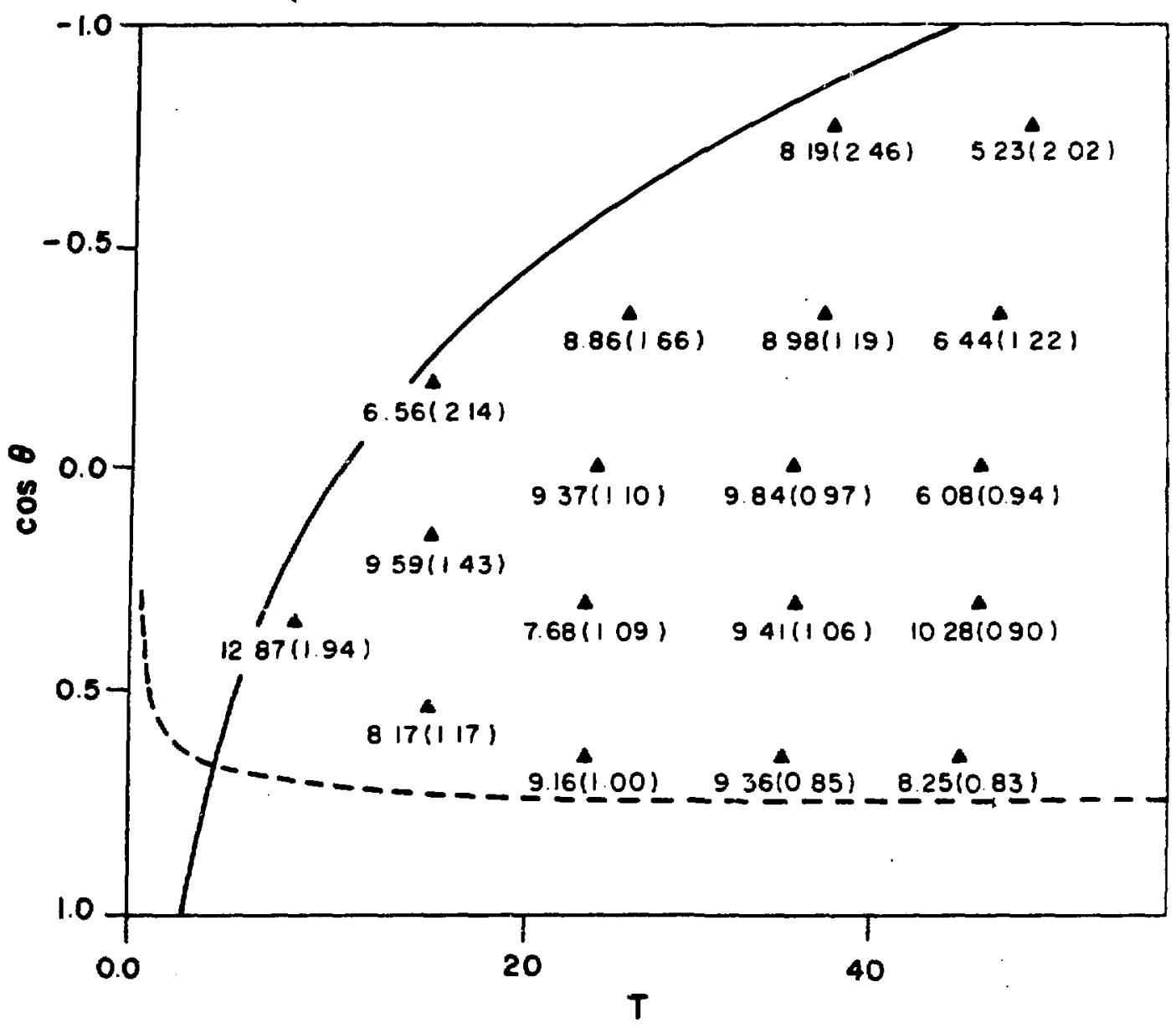

Fig. 31. Values of $\left\langle|M|^{2}\right\rangle$ at $254 \mathrm{MeV}$. The uncertainties are statistical. 


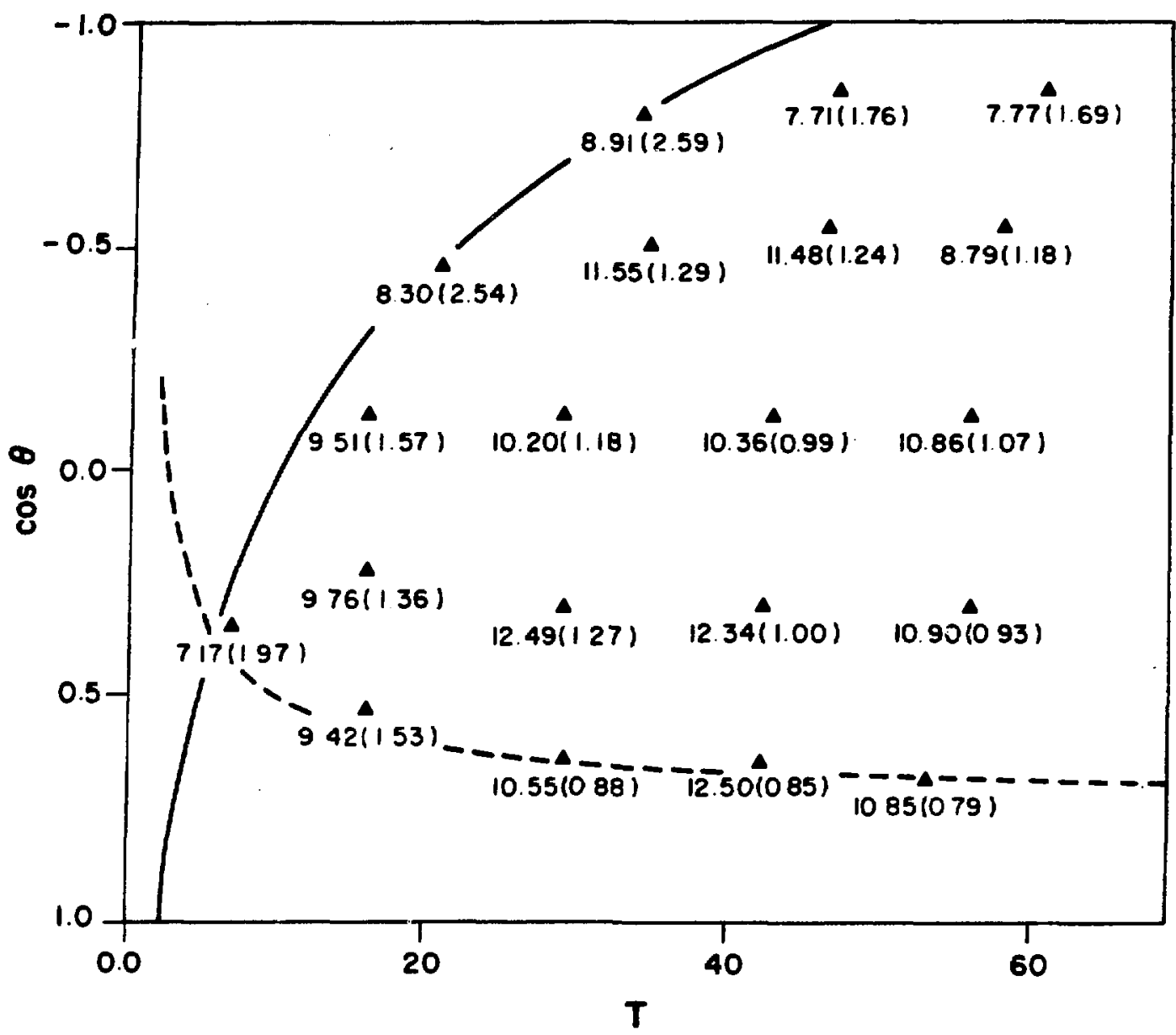

Fig. 32. Values of $\left\langle|M|^{2}>\right.$ at $279 \mathrm{MeV}$. The uncertainties are statistical. 


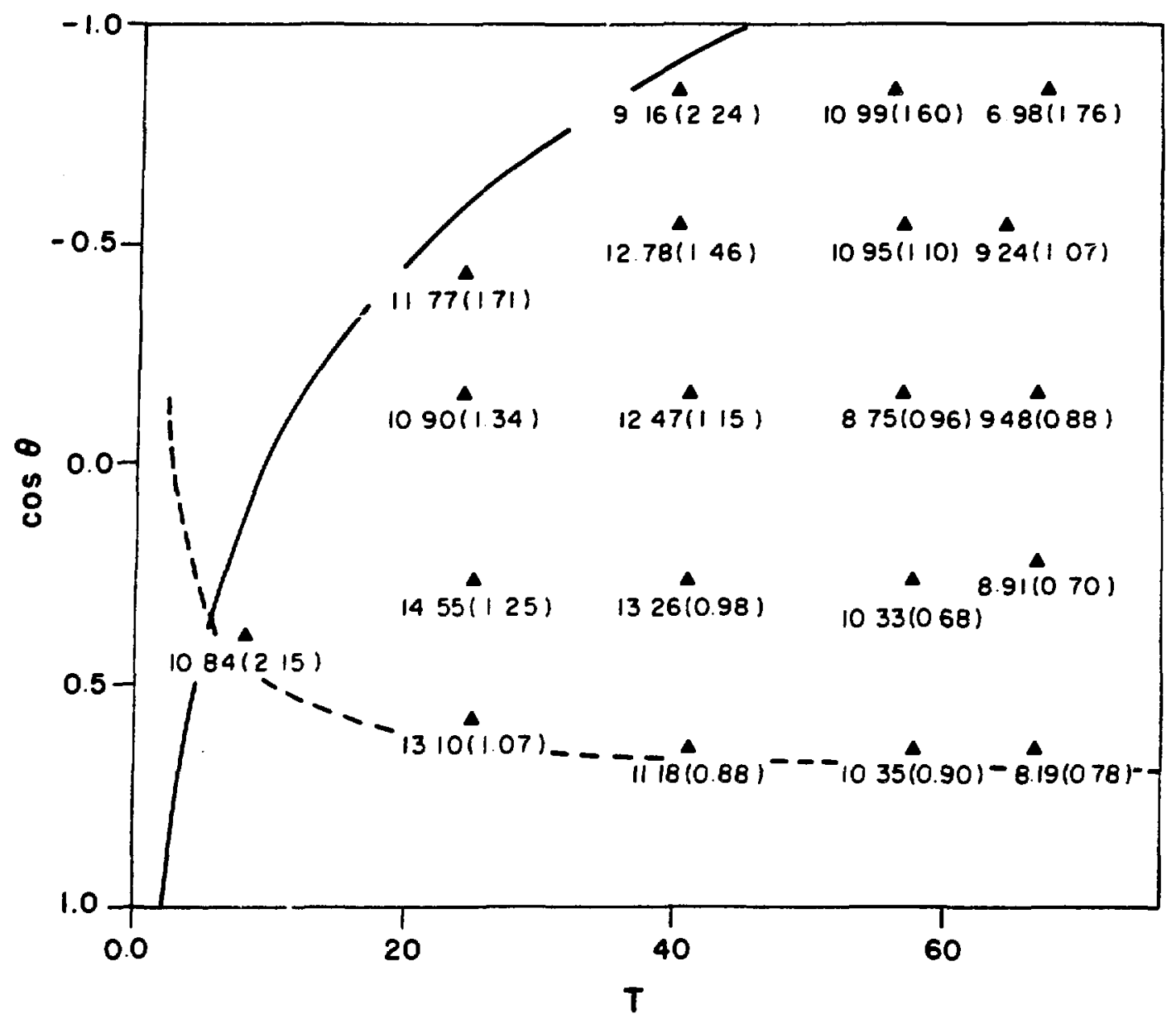

Fig. 33. Values of $\left\langle\left. M\right|^{2}>\right.$ ät $292 \mathrm{MeV}$. The uncertainties are statistical. 


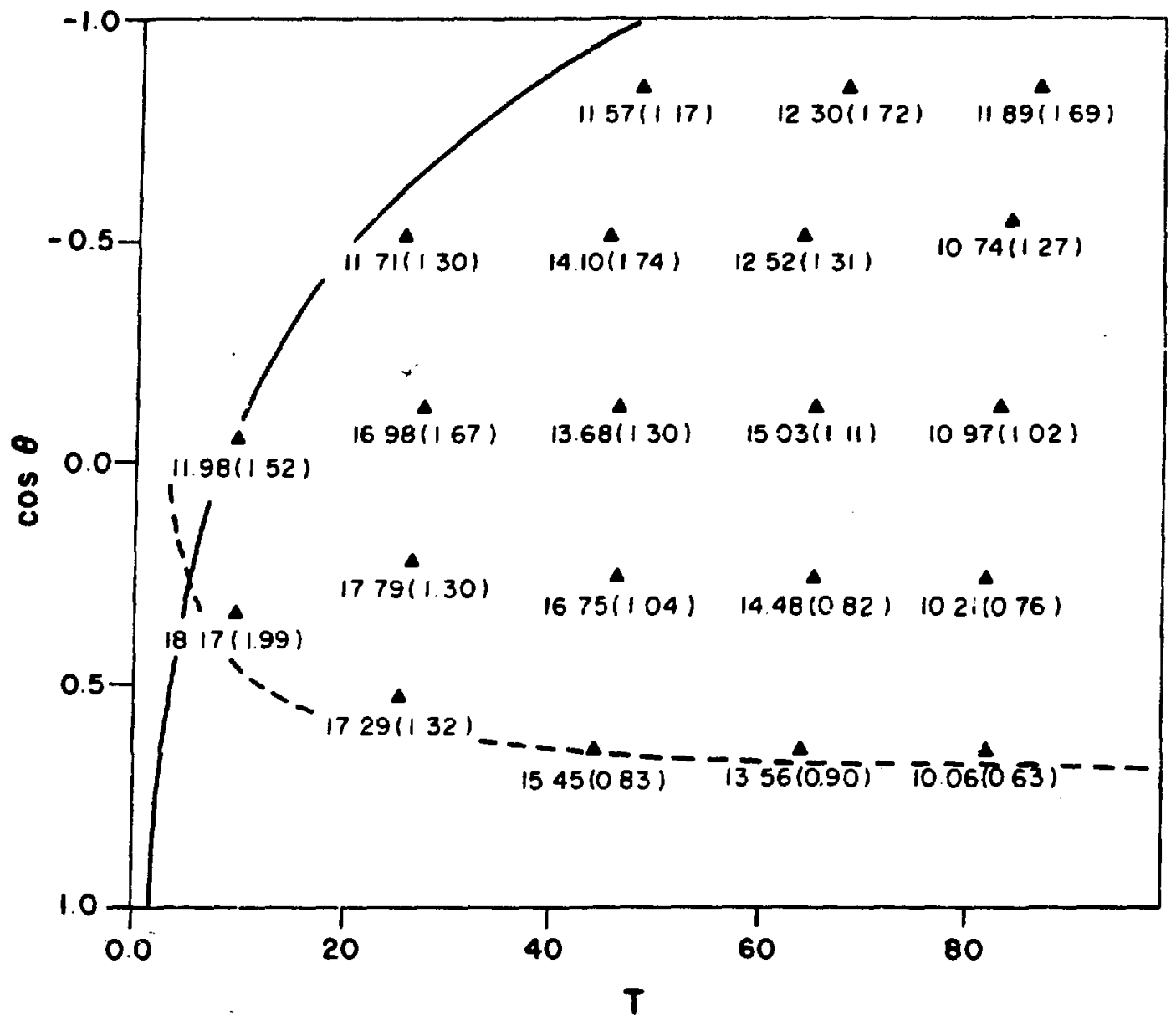

Fig. 34. Values of $\left\langle|M|^{2}\right\rangle$ at $330 \mathrm{MeV}$. The uncertainties are statistical. 


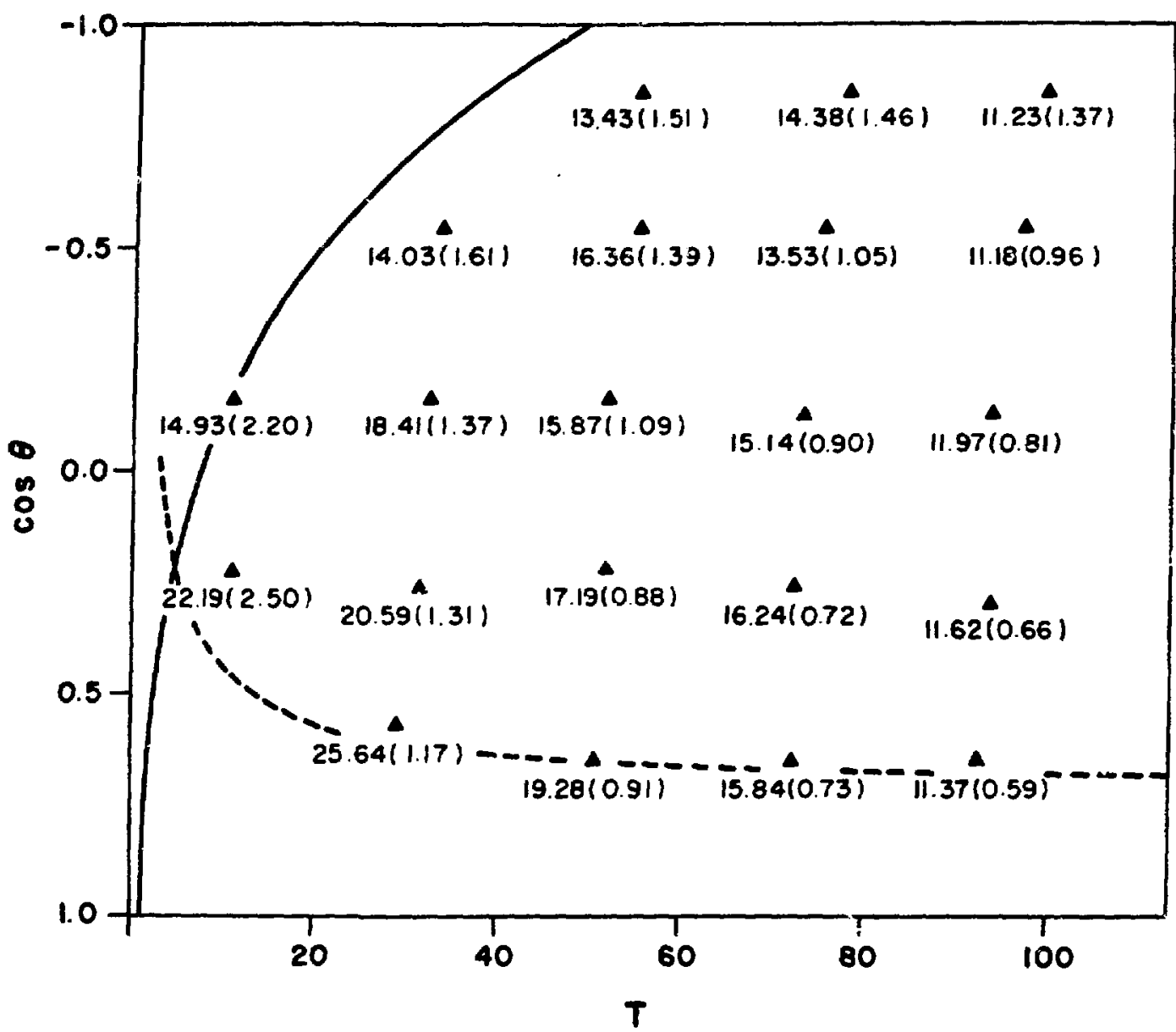

Fig. 35. Values of $\left\langle|M|^{2}>\right.$ at $356 \mathrm{MeV}$. The uncertainties are statistical. 


$$
x^{2}=\sum_{j}\left(\left\langle|M|^{2}\right\rangle_{j}-f\left(T_{j}, \cos \theta_{j}\right)\right)^{2} \Delta_{M j}^{-2}
$$

using the algorithm described in Appendix $A$. Table XXVIII presents the resulting pa:ameter values and the $x^{2} / v$ obtained for the five energies. $\zeta_{3}$ was required only for the two highest energies, and $\zeta_{11}$ for the three highest.

The integrated cross sections were calculated as

$$
\sigma_{R}=\int_{0}^{T_{\max }} \int_{-1}^{1} \int_{0}^{2 \pi} f(T, \cos \theta) \frac{d^{2} \sigma_{0}}{d \Omega d T} d \phi d \cos \theta d T \text {, }
$$

where the integrals over $\phi$ and $\cos \theta$ were carried out analytically while the integral over $T$ was computed numerically. The statistical uncertainty in $\sigma_{R}$ was calculated from the error matrix provided by the least squares algorithm. The uncertainty due to the uncertainty in $\varepsilon$ was again taken to be the deviation produced by the substitution of $\varepsilon \pm \Delta \varepsilon$ for $\varepsilon$. These results are presented in Table XXIX and ․: Fig. 36 along with a selection -f previous data. ${ }^{24-30}$ The errors in the figure include in quadrature the statistical uncertainty, the sensitivity to $\varepsilon$, and a normalization uncertainty of $3 \%$ at the lowest three energies and $4 \%$ at the higher two. The integrated cross sections are in reasonable agreement with previous results.

The integrated cross section corrected to exclude Coulomb enhancement was also calculated,

$$
\sigma_{R}^{\prime}=\int_{0}^{T_{\max }} \int_{-1}^{1} \int_{0}^{2 \pi} C^{-1}(T) f(T, \cos \theta) \frac{d^{2} \sigma_{0}}{d \Omega d T} d \phi d \cos \theta d T \text {. }
$$

The ratio $\overline{|M|^{2}}=\sigma_{R}^{\prime} / \sigma_{0}$ represents the square modulus of the matrix element correct,ed for Coulomb enhancement and averaged over phase space. It is $\overline{|M|^{2}}$ which is to be extrapolated to the $172 \mathrm{MeV}$ threshold, and 
Table XXVIII. Parameter values for $\left\langle|M|^{2}\right\rangle$ at each energy. Uncertainties include only statistics.

\begin{tabular}{lccccc}
\hline \hline$T$ & $\zeta_{1}$ & $\zeta_{2}$ & $\begin{array}{c}\zeta_{3} \\
(\mathrm{GeV})^{-1}\end{array}$ & $\begin{array}{c}\zeta_{4} \\
(\mathrm{GeV})^{-2}\end{array}$ & $x^{2} / \nu$ \\
\hline 254 & $2.85 \pm 0.06$ & $0.21 \pm 0.13$ & - & - & 1.43 \\
279 & $3.18 \pm 0.04$ & $0.20 \pm 0.09$ & - & - & 1.02 \\
292 & $3.63 \pm 0.08$ & $0.01 \pm 0.08$ & - & $-152 . \pm 25$. & 0.91 \\
330 & $3.93 \pm 0.07$ & $-0.42 \pm 0.16$ & $14.7 \pm 3.6$ & $-92 . \pm 15$. & 1.28 \\
356 & $4.39 \pm 0.06$ & $-0.43 \pm 0.13$ & $15.7 \pm 2.8$ & $-113 . \pm 10$. & 0.78 \\
\hline
\end{tabular}


Table XXIX. Integrated cross sections for $\pi^{-} p \rightarrow \pi^{+} \pi^{-} n . \Delta_{1}$ is the statistical uncertainty, while $\Delta_{2}$ is the overall uncertainty including allowances for normalization and Cerenkov efficiency uncortainties in quadrature. Units are MeV and $\mu \mathrm{b} / \mathrm{sr}$.

\begin{tabular}{cccc}
\hline$T$ & $\sigma_{R}$ & $\Delta_{1}$ & $\Delta_{2}$ \\
\hline 254.3 & 173.2 & 6.6 & 11.1 \\
279.3 & 380.3 & 10.5 & 17.1 \\
292.0 & 535.9 & 17.2 & 24.5 \\
330.8 & 1159. & 27. & 54. \\
355.9 & 1863. & 37. & 84. \\
\hline
\end{tabular}




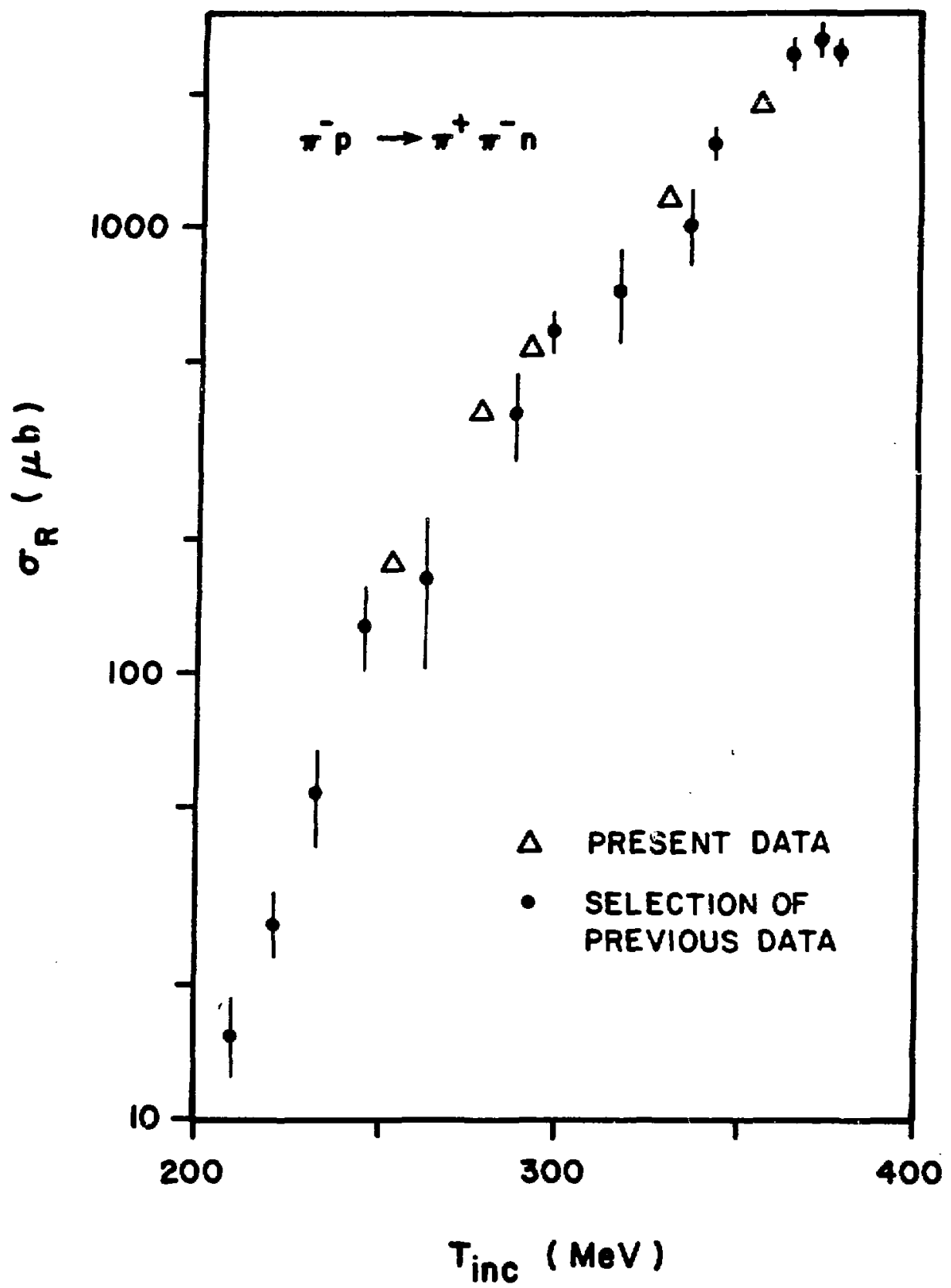

Fig. 36. Integrated cross sections for $\pi^{-} p \rightarrow \pi^{+} \pi^{-} n$ from present experiment and a selection of previous measurements. ${ }^{24-30}$ 
its root compared to the soft pion result in Table I.

\section{Comparison To Soft Pion Theory}

$\mid M^{2}$ was extrapolated to threshold from the five values from this experiment and a more recent value at $230 \mathrm{MeV} .^{3}$. The $\mid M T^{2}$ were fitted to

$$
f\left(T_{\text {tot }}\right)=S_{1}+S_{2} T_{\text {tot }} \text {, }
$$

where $T_{\text {tot }}$ is the total kinetic energy in the center of momentum frame and $S_{j}$ are adjustable parameters. The points and curve are shown in Fig. 37. Fitting to higher order polynominals does not appear to be warranted. The results when $\varepsilon$ was changed by $\pm \Delta \varepsilon$ are presented in Fig. 38, illustrating the sensitivity of the results to the efficiency for identifying the energetic positron background. The extrapolated theshold value of $|M|^{2}$ is fairly insensitive to $\varepsilon$. This is due to the inclusion of the value of $T M T^{2}$ at the higher energies.

The results of the extrapolation to threshold and the comparison to soft pion theory are given in Table $X X X$. The symmetry breaking parameter $\xi$, obtained from the equation in Table $I$, is clearly sensitive to the choice of sign for the threshold matrix element. Consequently, the implied predictions for the threshold matrix element for $\pi^{+} p \rightarrow \pi^{+} \pi^{+} n$ and $\pi^{-} p \rightarrow \pi^{0} \pi^{0} n$ (See Table I) are similarly sensitive to the choice of sign. The meager supply of data in these two reactions strongly favors the first choice ${ }^{2}$ in Table $x x x, M_{\text {threshold }}=-1.317 . \xi$ is also sensitive to $f_{\pi}$. The three values of $f_{\pi}$ in the table correspond to the Goldberger-Treiman value and the extremes from the other soft pion calculations. These suggest the possible variation between $f_{\pi}$ at the physical threshold and at the nonphysical soft pion limit. In any case 


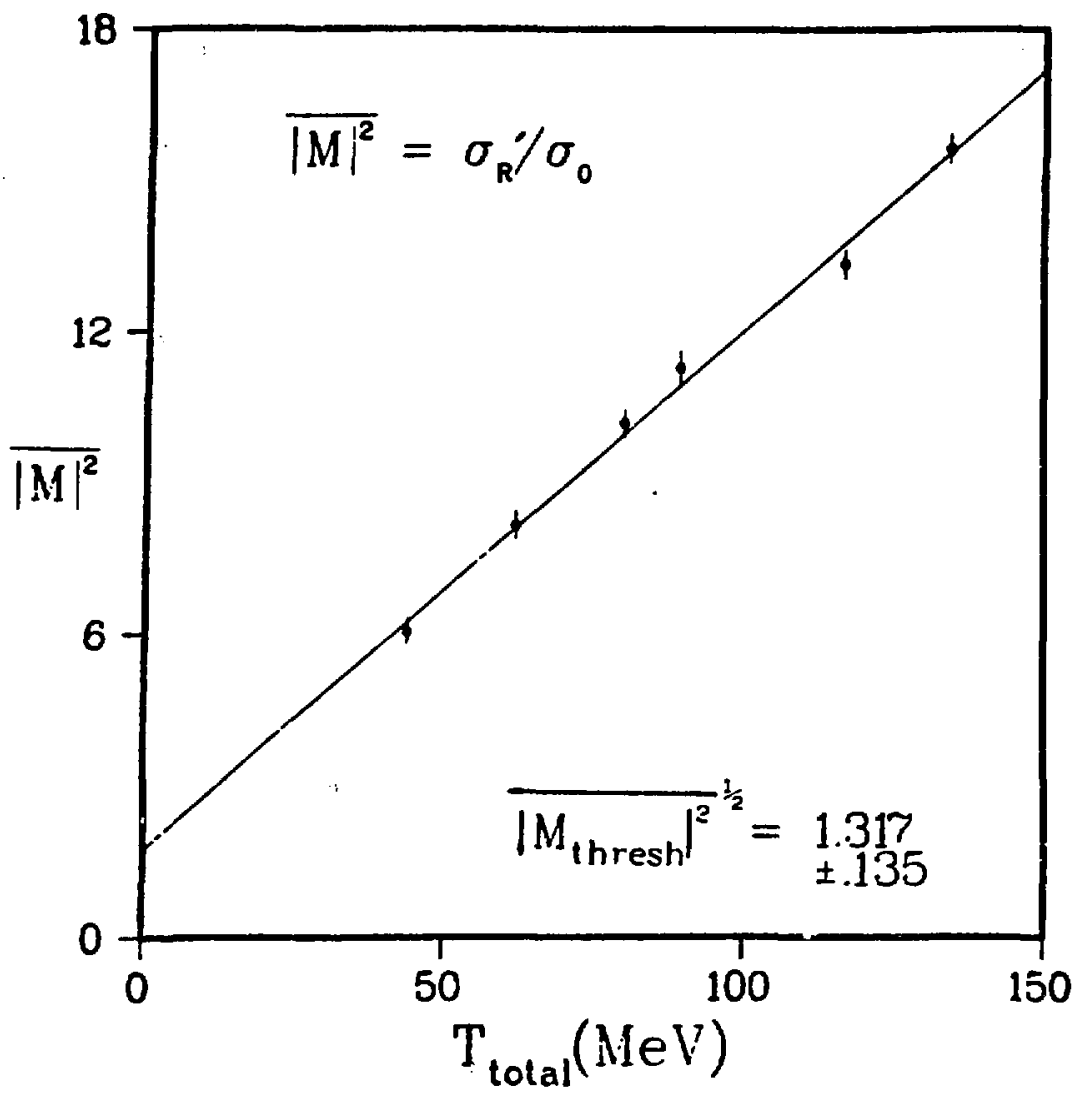

Fig. 37. Extrapolation of $|M|^{2}$ to threshold. The uncertainties are statisticai. 


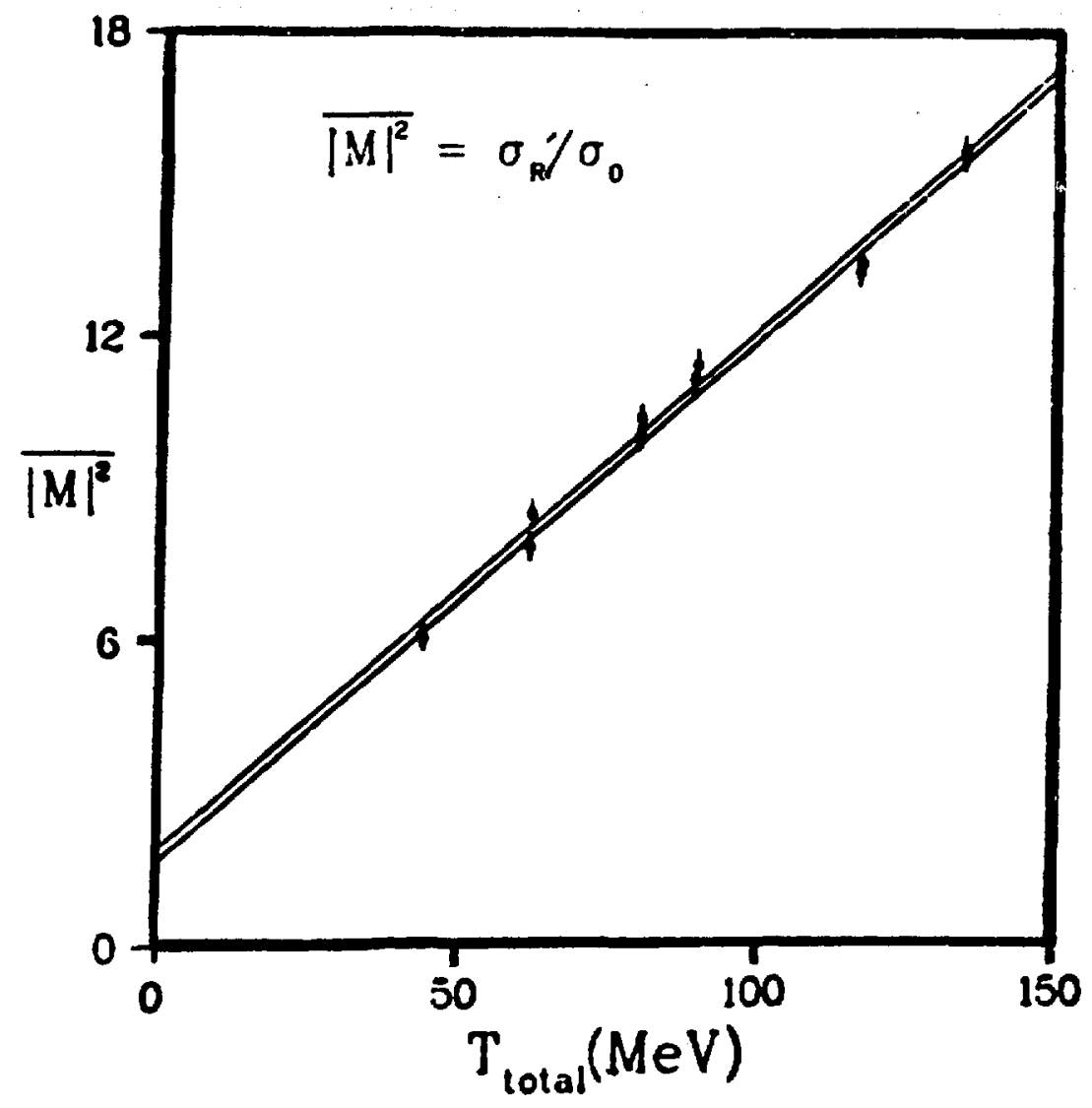

Fig. 38. Effect of the uncertainty in $\varepsilon$. The points and curve correspond to $\varepsilon$ replaced by $\varepsilon \pm \Delta \varepsilon$. 
Table $x x x$. Comparison to soft pion theory.

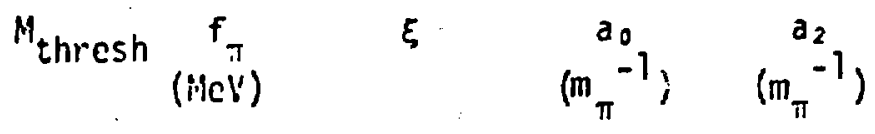

\begin{tabular}{|c|c|c|c|c|c|}
\hline Value & -1.268 & 86.9 & 0.15 & 0.170 & -0.055 \\
\hline Error & 0.202 & & 0.34 & 0.022 & 0.009 \\
\hline$\varepsilon$ sensitivity & 0.046 & & 0.08 & 0.005 & 0.002 \\
\hline Value & & 81.8 & 0.39 & 0.175 & -0.069 \\
\hline Error & & & 0.30 & 0.022 & 0.009 \\
\hline E sensitivity & & & 0.08 & 0.005 & 0.002 \\
\hline Value & & 94.0 & -0.21 & 0.165 & -0.039 \\
\hline Error & & & 0.39 & 0.022 & 0.009 \\
\hline$\varepsilon$ sensitivity & & & 0.10 & 0.005 & 0.002 \\
\hline Value & 1.268 & 86.9 & 4.38 & -0.101 & -0.164 \\
\hline Error & 0.202 & & 0.34 & 0.022 & 0.009 \\
\hline$\varepsilon$ sensitivity & 0.046 & & 0.08 & 0.005 & 0.002 \\
\hline Value & & 81.8 & 4.13 & -0.097 & -0.178 \\
\hline Error & & & 0.30 & 0.022 & 0.009 \\
\hline$\varepsilon$ sensitivity & & & 0.08 & 0.005 & 0.002 \\
\hline Value & & 94.0 & 4.73 & -0.106 & -0.148 \\
\hline Error & & & 0.39 & 0.022 & 0.009 \\
\hline$\varepsilon$ sensitivity & & & 0.10 & 0.005 & 0.002 \\
\hline
\end{tabular}


$\xi$ is consistent with zero. Presuming for the moment the validity of soft pion calculations, the $\pi-\pi$ s-wave scattering lengths can be calculated and are presented in the table for each value of $\xi$. The determination of $a_{0}$ from $k_{e^{4}}$ decay $\left(k^{+} \rightarrow \pi^{+} \pi^{-} e^{+} \nu_{e}\right)$ is $31 a_{0}=0.26 \pm 0.05 \mathrm{~m}_{\pi}^{-3}$. This clearly favors the choice $M_{\text {threshold }}<0$, but is somewhat larger than any of the results in the table. This is not a test of the soft pion calculation, since the $k_{e^{4}}$ measurement is difficult and its result is model dependent. 


\section{CHAPTER VI \\ SUMMARY AND CONCLUSIONS}

We have presented a systematic study of the reaction $\pi^{-} p \rightarrow \pi^{+} \pi^{-} n$ in the region near threshold. The doubly differential cross section $\frac{d^{2} \sigma}{d \delta} d T$ for the produced $\pi^{+}$was measured at about twenty points for each of five energies from $254 \mathrm{MeV}$ to $356 \mathrm{MeV}$. The accuracy of the measurements ranged between $9 \%$ and $39 \%$ at $254 \mathrm{MeV}$ and between $4.7 \%$ and $14.7 \%$ at 356 MeV. These are the first meaningful measurements of the doubly differential cross section in this energy range. At each energy the integrated cross section was derived from the measurements with an uncertainty of about $5 \%$. As seen in Fig. 36 , this represents a significant improvement. The mean square modulus of the matrix element was also determined at each energy. The value at the $172 \mathrm{MeV}$ threshold was extrapolated from the set of these five values and a more recent value at $230 \mathrm{MeV} .{ }^{3}$ Finally, the symmetry breaking parameter $\xi$ of soft pion theory was determined.

The energy dependence of the mean square modulus evident from this work demonstrates the futility of extracting $\xi$ from any single measurement of the cross section. Indeed, as can be seen in Fig. 37, only 1/3 of the cross section at $230 \mathrm{MeV}$ arises from soft pion contributions. The hard pion contribution to the reaction evidently comprises $2 / 3$ of the cross section. The determination of $\xi$ favors the symmetry breaking mechanism proposed by Weinberg $(\xi=0)$ over those suggested by Schwinger $(\xi=1$ and $\xi=2) .^{2}$ 


\section{ACKNOWLEDGEMENTS}

I would like to thank the Department of Physics and Astronomy, the University of Wyoming and the Los Alamos Scientific Laboratory for the facilities and services they have provided over the course of my graduate eciucation. I would also like to thank the personnel and staff of the Clinton P. Anderson Meson Physics Facility for their hospitality during my residence in LOS Alamos.

I wish to acknowledge the support of the Department of Energy, Division of High Energy and Nuclear Physics, under contract EY-75-5-02-2197. A002, which made this work and my stay in Los Alamos possible.

The advice, guidance and encouragement of Prof. Glen A. Rebka, ur. has been invaluable.

Thanks are due Peter Gram, Charles Bordner, Alden Oyer, Mark Manley and Chris Bjork for valuable and interesting discussions. I thank Maggie Eutsler, Judy Powers, Alden Oyer and my wife, Mary Jean for their assistance in typing and preparing various drafts of this dissertation. The latter two are due special thanks as they provided help and encouragement which only the truest friends could have offered.

Finally, I dedicate this work to Daniel, my son, and Joseph, my father. To life. 


\section{APPENDIX A}

\section{NONLINEAR LEAST SQUARES FITTING ALGORITHM}

The fitting algorithm minimizes the quantity

$$
x^{2}=\sum_{i} \sigma_{i}^{-2}\left\{y_{i}-f\left(x_{i}, \vec{\zeta}\right)\right\}^{2}
$$

where $\left(x_{j}, y_{j}\right)$ is the $i$ th data point, $v_{j}$ is the uncertainty in $y_{j}$ and $\vec{\zeta}$ represents the collection of adjustable parameters. The function $f$ can be any function which exists and has a derivative with respect to each of the $\zeta_{j}$ at each of the data points, and is in general nonlinear in the parameters $\zeta$. Based upon the Gauss or Taylor series method, the algorithm seeks a root to

$$
\nabla_{\zeta} \chi^{2}=0 \text {, }
$$

where $\nabla_{\zeta}$ is the gradient with respect to the parameters. The method makes the linearizing assumption

$$
\nabla_{\zeta} x^{2}=\bar{A}_{\vec{\zeta}} . \quad\left(\bar{\zeta}_{\bar{\zeta}}-\vec{\zeta}^{\star}\right),
$$

where $\vec{\zeta}^{\star}$ is the collection of the optimum parameter values and

$$
\bar{A}_{\vec{\zeta}}=2 \sum_{i}\left\{\nabla_{\zeta} f\left(x_{i}, \vec{\zeta}\right)\right\} \sigma_{i}^{-2}\left\{\nabla_{\zeta} f\left(x_{i}, \vec{\zeta}\right)\right\} .
$$

This expression for $\nabla_{\zeta} x^{2}$ would be the first term of the Taylor expansion about $\vec{\zeta}^{\star}$ if the gradients in $\bar{A}$ could be evaluated at $\bar{\zeta}^{*}$. Not knowing $\vec{\zeta}^{\star}$, the above expression for $\nabla_{\zeta} \chi^{2}$ is used.

The solution is then sought in an iterative fashion. An initial guess $\vec{\zeta}$ is made for $\vec{\zeta}^{\star}, \nabla x^{2}$ and $\bar{A}$ are calculated for this $\vec{\zeta}$, and the set of equations relating $\nabla x^{2}$ to $\bar{A}$ are solved to obtain a new guess for $\vec{\zeta}^{\star}$. This procedure is repeated until the resu?tant change in each of 
the parameters is less than some standard, in our case one part in $10^{\circ}$. This method can be used to find the roots of any nonlinear equation and is always plagued with the same difficulties. In particular if the initial guess is too far from the desired solution, the algorithm may converge to an undesired root which may actually correspond to a relative maximum in $x^{2}$, or it may fail to converge entirely. For the applications in this work, the method converged in fifteen iterations or less when the initial guess was reasonable. Erroneous solutions were obvious. The algorithm also provides an error matrix. The matrix $A$ is the inverse of the error matrix when $f(x, \vec{\zeta})$ is linear in each of the $\zeta_{j}$ and the $y_{j}$ possess Gaussian statistics. That is, if the parameter $\zeta_{j}$ is displaced from $\zeta_{j}{ }^{*}$ by $\pm\left\{\left(A^{-1}\right)_{j j}\right\}^{\frac{1}{2}}$ and the remaining $\zeta_{i}$ are varied to minimize $x^{2}$ under that constraint, the resulting minimum yalue will be larger than the unconstrained minimum by 1 . For a nonlinear function $f(x, \vec{\zeta})$, the error matrix provided by the algorithm is the linear approximation. 


\section{APPENDIX B}

MOMENTUM CORRECTIONS

Several corrections were included in the determination of the momentum distribution of the pions in each of the incident beams. Two corrections were made to the centroid of the distribution and four corrections to the width of the distribution.

The first correction ploss to the momentum of the scattered pions accounted for loss of energy to atomic electrons

$$
-p_{\text {loss }}=\frac{d p_{\text {out }}}{d p_{\text {in }}} \frac{d p_{\text {in }}}{d x} \overline{S_{\text {in }}}+\frac{d p_{\text {out }}}{d x} \overline{S_{\text {out }}} \text {. }
$$

Here $p_{\text {in }}$ is the incident momentuin, $p_{\text {out }}$ is the scattered momentum calculated from two body kineriatics, and $\overline{S_{\text {in }}}$ and $\overline{S_{\text {out }}}$ are the mean path lengths entering and leaving the target. The momentum loss $\frac{d p}{d x}$ was based upon the Bethe-Block formula

$$
-\frac{1}{\rho} \frac{d E}{d x}=\frac{2 \pi n e^{4}}{\rho m_{e} \beta}\left\{\ln \left(\frac{2 m_{e} \beta^{2} W_{\max }}{I^{2}\left(1-\beta^{2}\right)}\right)-2 \beta^{2}-\delta-U\right\}
$$

where $E$ and $B$ are the energy and velocity of the particle, $\rho$ is the density of the material, $n$ is the number density of the electrons in the material, e and $m_{e}$ are the charge and mass of the electron, and $W_{\max }$ is the maximum energy which can be transferred to an electron in a single collision. The ionization potential I of hydrogen is $18.30 \mathrm{eV} .^{32}$ The density correction $\delta$ was calculated according to the formulae of Sternheimer. ${ }^{33}$ The shell correction U was negligible, being important only when $\beta<\beta_{\mathrm{e}}$ with $\mathrm{B}_{\mathrm{e}}$ the velocity of the atomic electron. 
The second correction $p_{\text {ang }}$ to the momentum of the scattered pior.s allowed for the finite angular acceptance of the spectrometer and the finite emittance of the pion channel. The mean cosine of the scattering angle differed from the cosine of the mean scattering angle, for which Pout was calculated. Thus $\mathrm{P}_{\text {out }}$ was corrected by

$$
p_{\text {ang }}=\left(\overline{\cos \theta}-\cos \theta_{0}\right) \frac{d p_{\text {Qut }}}{d \cos \theta},
$$

where all quantities are in the laboratory frame.

of the four corrections to the width of the scattered distribution, two were for variation in loss of energy and two for variation in scattering angle. The dominant correction for variation in loss of energy was from variation in path lengths in the target, and was given by

$$
\begin{aligned}
\sigma_{\text {loss }}^{2} & =\left(\overline{S_{\text {in }}^{2}}-{\overline{S_{\text {in }}}}^{2}\right)\left(\frac{d p_{\text {oui }}}{d p_{\text {in }}}\right)^{2}\left(\frac{d p_{\text {in }}}{d x}\right)^{2}+\left(\overline{S_{\text {out }}^{2}}-\overline{S_{\text {out }}}\right)\left(\frac{d p_{\text {out }}}{d x}\right)^{2} \\
& +2\left(\overline{S_{\text {in }} S_{\text {out }}}-\overline{S_{\text {in }}} \overline{S_{\text {out }}}\right)\left(\frac{d p_{\text {out }}}{d x}\right)\left(\frac{d p_{\text {out }}}{d p_{\text {in }}}\right)\left(\frac{d p_{\text {in }}}{d x}\right) .
\end{aligned}
$$

The random natu:e of the collisions also provided a variation to the energy lost to atomic electrons. The mean square contribution was

$$
\sigma_{\text {strag }}^{2}=\overline{S_{\text {out }}} \frac{d}{d x} \overline{(p-\bar{p})^{2}}=\overline{S_{\text {out }}} \frac{2 \pi e^{4} n\left(1+\gamma^{2}\right)}{1+2 \gamma\left(m_{e} / m_{\pi}\right)+\left(m_{e} / m_{\pi}\right)^{2}}
$$

where $n$ is the number density of the electrons. The dominant correction for variation in angle was due to the geometry of the system, and was given by

$$
\left.\sigma_{\text {ang }}^{2}=-\overline{\left(\cos ^{2} \theta\right.}-\overline{\cos \theta^{2}}\right)\left(\frac{d \text { pout }}{d x}\right)^{2} .
$$

A correction for small angle scattering was also included. Its mean 
square contribution was

$$
\sigma_{\text {cout }}^{2}=\frac{\overline{S_{\text {Out }}} E_{S}^{2}}{X_{0} P_{\text {out }}^{2} \beta^{2}}\left(\frac{d \text { pout }}{d \theta}\right)^{2}
$$

where $x_{0}$ is the radiation length in liquid hydrogen, and $E_{S}=m_{e}\left(\frac{4 \pi}{\alpha}\right)^{\frac{1}{2}}$. 


\section{APPENDIX C $\mathrm{e}^{+}$VETO EFFICIENCY}

The background for the investigated reaction $\pi^{-} p \rightarrow \pi^{+} \pi^{-} n$ included a spectrum of $e^{+}$. This background resulted from the sequence of single charge exchange in the target wall and hydrogen, $\pi^{\circ}$ decay, and pair production in the spectrometer with the $\mathrm{e}^{+}$transported to the surface barrier detectors. The ability to el iminate these events was crucial since they were largely associated with the liquid hydrogen, and hence not corrected by the subtraction of the target empty rate. These $\mathrm{e}^{+}$events were principally identified with the threshold Cerenkov detector described in Chapter II. This Appendix deals with the efficiency of that device.

The efficiency was found to be substantially less than 1.0 when the integrated cross section was remeasured at the lowest energy. Assuming an efficiency of 1.0, the present experiment determined $\sigma_{R}=93 \pm 6 \mu \mathrm{b}$ at $229 \mathrm{MeV}$, while the remeasurement ${ }^{3}$ yielded $\sigma_{R}=60 \pm 3 \mu \mathrm{b}$ at $230 \mathrm{MeV}$. A careful reexamination of both experiments left no doubt that the electron efficiency of the Cerenkov detector in this experiment was at fauit. The spectra of energetic pions and electrons detected by the Cerenkov detector of the remeasurement (which also used $F C-88$ as the medium, but provided more uniform trajectories) and the fraction of energetic pions detected by the Cerenkov detector in this experiment (available from the elastic scattering data) suggested an efficiency no greater than $88 \%$. 
The efficiency was determined from the $\pi^{+}$production data taken at $229 \mathrm{MeV}$ in this experiment and the remeasurement at $230 \mathrm{MeV}$ in an iterative fashion. A guess was made for $\varepsilon$, the efficiency, and $\overline{|M|^{2}}$ was calculated from the data at $229 \mathrm{MeV}$, where $\overline{|M|^{2}}$ is the mean square modulus of the matrix element corrected for Coulomb enhancement. This value was required to agree with $\overline{\left|M_{0}\right|^{2}}$, the value of $\overline{|M|^{2}}$ determined from the remeasurement at $230 \mathrm{MeV}$. Values were tried for $\varepsilon$ until this equirement was met to within one part in 600 . Forming a $x^{2}$

$$
x^{2}=\frac{\left(\overline{\left|M_{0}\right|^{2}}-\overline{|M(\varepsilon)|^{2}}\right)^{2}}{\Delta_{M_{0}}^{2}+\Delta_{M}^{2}}
$$

where $\Delta_{M_{0}}$ is the statistical uncertainty in $\overline{\left|M_{0}\right|^{2}}$ and $\Delta_{M}$ is the statistical uncertainty in $\overline{|M(\varepsilon)|^{2}}$ for the optimum value of $\varepsilon$. The uncertainty in $\varepsilon$ was taken to be the variation in $\varepsilon$ which produced $x^{2}=1$. The resultant Cerenkov efficiency for electrons was $\varepsilon=0.828 \pm 0.038$.

The above efficiency applied for nearly all of the data, but not for the bulk of the data at $292 \mathrm{MeV}$. This data was chronologically first, and for the most part was taken with the $S_{3}$ trigger scintillator near $S_{1}$ and $S_{2}$ instead of near the Cerenkov detector. The threshold level on the Cerenkov detector was also different in the early configuration. The electron efficiency of the Cerenkov detector $\varepsilon_{e}$ for the early data was thus different from the efficiency for the rest of the data. Four of the data points at $292 \mathrm{MeV}$ were measured with both trigger configurations and hence both efficiencies. These points provided a basis for determining $\varepsilon_{e}$. A $\chi^{2}$ was defined

$$
x^{2}=\sum_{i=1}^{4} \frac{\left\{\left\langle|M|^{2}\right\rangle_{i}-\left\langle|M|^{2} \geq e i\right\}^{2}\right.}{\Delta_{M i}^{2}+\Delta_{M e i}^{2}}
$$


with the subscript e denoting the results from the early configuration. The $\left\langle|M|^{2}\right\rangle_{i}$ were calculated for the value of $\varepsilon$ determined above, and $x^{2}$ was calculated for seven values of $\varepsilon_{e}$ near the optimum value. The values of $x^{2}$ were well fitted by a parabola as a function of $x=\left(1-\varepsilon_{e}\right) / \varepsilon_{e}$. The optimum $\varepsilon_{e}$ was associated with the minimum of the parabola, while its uncertainty was identified with the variation in $\varepsilon_{e}$ which increased $\chi^{2}$ by 1.0 over its minimum value. The resulting value was $\varepsilon_{e}=0.653 \pm$ 0.28 . 


\section{REFERENCES}

1. M. G. Olsson and Leaf Turner, Phys. Rev. 181, 2141 (1969).

F or $g_{v} / g_{a}=1.253$ the Goldberger-Treiman relation gives $f_{\pi}=86.9$ $\mathrm{MeV}$. Various determinations of the $\pi \mathrm{N}$ s-wave scattering lengths (see M. M. Nagels et al., Nucl. Phys. B147, 189 (1979)) give results between $82 \mathrm{MeV}$ and $92 \mathrm{MeV}$.

2. Leaf Turner, Ph.D. dissertation, University of Wiscons in, 1969 (unpubtished).

3. C. W. Bjork, S. E. Jones, T. R. King, D. M. Manley, A. T. Oyer, G. A. Rebka, Jr., J. B. Walter, R. Carawon, P. A. M. Gram, F. T. Shively, C. A. Bordner and E. L. Lomon, to be published.

4. $P^{3}$ User Group and P. A. M. Gram, "A Proposal for P3, A Versatile High-Energy Pion Beani Facility", LASL Report \# LA-4535-MS, 1970; H. H. Howard, B. Storms and S. P. Slatkin, LAMPF Users Handbook, (Los Alamos Scientific Laboratory, Medium Energy Physics Division, Los Alamos, NM, 1974).

5. The necessary magnetic fields were determined in a TRANSPORT simulation and converted to shunt voltages by SHUNT. K. L. Brown, "A First and Second Order Matrix Theory for the Design of Beam Transport Systems and Charged Particle Spectrometers," SLAC-75 (1967); R. Macek, private communication, 1973.

6. G. J. Krausse and P. A. M. Gram, Nucl. Instr. and Meth. 156, 365 (1978).

7. The target flask was positioned with the aid of a surveyor's transit. The precision obtained was about $0.5 \mathrm{~mm}$ after the target was subsequentiy cooled down to liquid hydrogen temperatures.

8. A. T. Oyer, Ph.D. dissertation, University of Wyoming, 1976. Published as LASL Report \#LA-6599-T.

9. B. Grennberg and A. Rytz, Metrologia 7,65 (1971); CRC Handbook of Chemistry and Physics, 59th ed. (CRC Press, inc., West Palm Beach, Florida, 1979), p. B-353.

10. J. B. Walter and G. A. Rebka, Jr., "SCATPI, A Subroutine for Calculating $\pi \mathrm{N}$ Cross Sections and Polarizations for Incident Pion Kinetic Energies Between 90 and 300 MeV", LASL Report "LA-7731-MS, 1979.

11. J. R. Carter, D. V. Bugg and A. A. Carter, Nucl. Phys. B58, 378 (1973). 
12. P. J. Bussey, J. R. Carter, D. R. Dance, D. V. Bugg, A. A. Carter and A. M. Smith, Nuc1. Phys. B58, 363 (1973).

13. A. A. Carter, J. R. Williams, D. V. Bugg, P. J. Bussey and D. R. Dance, Nucl. Phys. B26, 445 (1971).

14. D. V. Bugg, P. J. Bussey, D. R. Dance, A. R. Smith, A. A. Carter and J. R. Williams, Nucl. Phys. B26, 588 (1971).

15. E. H. Rogers, O. Chamberlain, J. H. Foote, H. M. Steiner, C. Wiegand and T. Ypsilantis, Rev. Mod. Phys. 33, 356 (1961).

16. J. H. Foote, O. Chamberlain, E. H. Rogers, H. M. Steiner, C. Wiegand and T. Ypsilantis, Phys. Rev. 122, 948 (1961).

17. L. D. Roper, R. M. Wright and B. T. Feld, Phys. Rev. 138, B190 (1964).

18. P. R. Auvi1, Phys. Rev. 168, 1568 (1968); D4, 240 (1971).

19. J. R. Taylor, Scattering Theory (Wiley \& Sons, New York, 1972), p. 213-231.

20. J. D. Jackson, Nuovo Cimento 34,1644 (1964).

21. V. Chaloupka et al., Phys. Lett. 50B, 10 (1974).

22. P. M. Ogden, D. E. Hagge, J. A. Helland, M. Banner, J. F. Detoeaf and J. Teiger, Phys. Rev. 137, B1715 (7965)

23. H. R. Rugge and 0. T. Vik, Phys. Rev. 129, 2300 (1963).

24. B. C. Barish, R. J. Kurz, V. Perez-Mendez and J. Solomon, Phys. Rev. 135, B416 (1964).

25. Yu A. Batusov et a1., Sov. J. Nuc1. Phys. 1, 374 (1965).

26. T. Blokhintseva et al., Proc. of the 12th Annual International Conference on High Energy Physics, Dubna (1964).

27. J. A. Jones, W. W. M. Allison and D. H. Saxon, Nucl. Phys. B83, $93(1974)$.

28. J. Kirz, J. Schwartz and R. D. Tripp, Phys. Rev. 130, 2481 (1963).

29. W. A. Perkins, III et a1., Phys. Rev. 118, 1364 (1960).

30. D. H. Saxon, H. H. Mutvey and W. Chinowsky, Phys. Rev. D2, 1790 (1970).

31. W. Ochs, AIP Conf. Proc. \#41, p. 326 (1978). 
32. J. Jani, Air Force Weapons Laboratory TR-65-150.

33. R. M. Sternheimer, Phys. Rev. 103, 511 (1956). 